\title{
Nematicidal Stemona Alkaloids from Stemona parviflora
}

Sheng-Zhuo Huang, Fan-Dong Kong, Qing-Yun Ma, Zhi-Kai Guo, Li-Man Zhou, Qi

Wang, Hao-Fu Dai, ${ }^{*}$ and You-Xing Zhao*

Key Laboratory of Biology and Genetic Resources of Tropical Crops, Ministry of Agriculture, Institute of Tropical Bioscience and Biotechnology, Chinese Academy of Tropical Agriculture Sciences, Haikou 571101, People's Republic of China

* Tel/Fax (H.F. Dai): +86-898-66961869. E-mail: daihaofu@itbb.org.cn.

*Tel/Fax (Y.X. Zhao): +86-898-66989095. E-mail: zhaoyouxing@itbb.org.cn.

\section{Supporting Information}

\section{S1: EXPERIMENTAL SECTION}

Theory and Calculation Details HPLC Map

Table S2 Nematicidal activity with RDR of isolated compounds

$\mathrm{S} 2$ : Figure $\mathrm{S} 1-\mathrm{S} 3$

S3: ${ }^{1} \mathrm{H}$ NMR of $3 \beta$ - $n$-butylstemonamine (1)

S4: DEPT of $3 \beta$ - $n$-butylstemonamine (1)

S5: HSQC of $3 \beta$ - $n$-butylstemonamine (1)

S6: HMBC of $3 \beta$ - $n$-butylstemonamine (1)

S7: ${ }^{1} \mathrm{H}^{1} \mathrm{H}$ COSY of $3 \beta$ - $n$-butylstemonamine

S8: ROESY of $3 \beta$ - $n$-butylstemonamine (1)

S9: ESIMS of $3 \beta$ - $n$-butylstemonamine (1)

S10: ${ }^{1} \mathrm{H}$ NMR of 8 -oxo- $3 \beta-n$ -

butylstemonamine (2)

S11: DEPT of 8-oxo-3 $\beta$-n-butylstemonamine (2)

S12: HSQC of 8-oxo-3 $\beta$ - $n$-butylstemonamine

S13: HMBC of 8-oxo-3 $\beta$-n-butylstemonamine

(2)
S14: ${ }^{1} \mathrm{H}{ }^{1} \mathrm{H}$ COSY of 8-oxo-3 $\beta$-n-

butylstemonamine (2)

S15: ROESY of 8-oxo-3 $\beta-n-$

butylstemonamine (2)

S16: ESIMS of 8-oxo-3 $\beta$ - $n$-butylstemonamine

(2)

S17: ${ }^{1} \mathrm{H}$ NMR of 3- $n$-butylneostemonine (3)

S18: DEPT of 3- $n$-butylneostemonine (3)

S19: HSQC of 3- $n$-butylneostemonine (3)

S20: HMBC of 3- $n$-butylneostemonine (3)

S21: ${ }^{1} \mathrm{H}^{1} \mathrm{H}$ COSY of 3- $n$-butylneostemonine

(3)

S22: ROESY of 3- $n$-butylneostemonine (3)

S23: ESIMS of 3- $n$-butylneostemonine (3)

S24: ${ }^{1} \mathrm{H}$ NMR of $10-e p i-3-n-$ butylneostemonine (4)

S25: DEPT of 10-epi-3-n-butylneostemonine

(4)

S26: HSQC of 10-epi-3-n-butylneostemonine

(4)

S27: HMBC of 10-epi-3-n-butylneostemonine

(4) 
S28: ${ }^{1} \mathrm{H}{ }^{1} \mathrm{H}$ COSY of $10-e p i-3-n-$

butylneostemonine (4)

S29: ROESY of 10-epi-3-n-

butylneostemonine (4)

S30: ESIMS of 10-epi-3-n-butylneostemonine

(4)

S31: ${ }^{1} \mathrm{H}$ NMR of 8-oxo-oxymaistemonine (5)

S32: DEPT of 8-oxo-oxymaistemonine (5)

S33: HSQC of 8-oxo-oxymaistemonine (5)

S34: HMBC of 8-oxo-oxymaistemonine (5)

S35: ${ }^{1} \mathrm{H}{ }^{1} \mathrm{H}$ COSY of 8-oxo-oxymaistemonine

S36: ROESY of 8-oxo-oxymaistemonine (5)

S37: ESIMS of 8-oxo-oxymaistemonine (5)

S38: ${ }^{1} \mathrm{H}$ NMR of protostemonine $N_{4}$-oxide (6)

S39: DEPT of protostemonine $N_{4}$-oxide (6)

S40: HSQC of protostemonine $N_{4}$-oxide (6)

S41: HMBC of protostemonine $N_{4}$-oxide (6)

$\mathrm{S} 42:{ }^{1} \mathrm{H}{ }^{1} \mathrm{H} \mathrm{COSY}$ of protostemonine $N_{4}$-oxide

(6)

S43: ROESY of protostemonine $N_{4}$-oxide (6)

S44: ESIMS of protostemonine $N_{4}$-oxide (6)
S45: ${ }^{1} \mathrm{H}$ NMR of (19S)-hydroxy-21methoxystemofoline (7)

S46: DEPT of (19S)-hydroxy-21methoxystemofoline (7)

S47: HSQC of (19S)-hydroxy-21methoxystemofoline (7)

S48: HMBC of (19S)-hydroxy-21methoxystemofoline (7)

S49: ${ }^{1} \mathrm{H}^{1} \mathrm{H}$ COSY of (19S)-hydroxy-21methoxystemofoline (7)

S50: ROESY of (19S)-hydroxy-21methoxystemofoline (7)

S51: ESIMS of (19S)-hydroxy-21methoxystemofoline (7)

S52: ${ }^{1} \mathrm{H}$ NMR of parvistemonine A (8)

S53: DEPT of parvistemonine A (8)

S54: HSQC of parvistemonine A (8)

S55: HMBC of parvistemonine A (8)

S56: ${ }^{1} \mathrm{H}^{1} \mathrm{H}$ COSY of parvistemonine A (8)

S57: ROESY of parvistemonine A (8)

S58: ESIMS of parvistemonine A (8)

S59: ECD of parvistemonine A (8) 


\section{S1 EXPERIMENTAL SECTION}

\section{Theory and Calculation Details}

The calculations were performed by using the density functional theory (DFT) as carried out in the Gaussian 03. ${ }^{1}$ The preliminary conformational distributions search (Table 1) was performed by HyperChem 7.5 software. All ground-state geometries were further optimized at the B3LYP/6$31 \mathrm{G}$ (d) level. Conformers within a $2 \mathrm{kcal} / \mathrm{mol}$ energy threshold from the global minimum were selected to calculate the electronic transitions. ${ }^{2}$ The overall theoretical ECD spectra were obtained according to the Boltzmann weighting of each conformers. Solvent effects of methanol solution were evaluated at the same DFT level by using the SCRF/PCM method. ${ }^{3}$

1. Gaussian 03, Revision E.01, M. J. Frisch, G. W. Trucks, H. B. Schlegel, G. E. Scuseria, M. A. Robb, J. R. Cheeseman, J. A. Montgomery, Jr., T. Vreven, K. N. Kudin, J.C. Burant, J. M. Millam, S. S. Iyengar, J. Tomasi, V. Barone, B. Mennucci, M. Cossi, G. Scalmani, N. Rega, G. A. Petersson, H. Nakatsuji, M. Hada, M. Ehara, K. Toyota, R. Fukuda, J. Hasegawa, M. Ishida, T. Nakajima, Y. Honda, O. Kitao, H. Nakai, M. Klene, X. Li, J. E. Knox, H. P. Hratchian, J. B. Cross, V. Bakken, C. Adamo, J. Jaramillo, R. Gomperts, R. E. Stratmann, O. Yazyev, A. J. Austin, R. Cammi, C. Pomelli, J. W. Ochterski, P. Y. Ayala, K. Morokuma, G. A. Voth, P. Salvador, J. J. Dannenberg, V. G. Zakrzewski, S. Dapprich, A. D. Daniels, M. C. Strain, O. Farkas, D. K. Malick, A. D. Rabuck, K. Raghavachari, J. B. Foresman, J. V. Ortiz, Q. Cui, A. G. Baboul, S. Clifford, J. Cioslowski, B. B. Stefanov, G. Liu, A. Liashenko, P. Piskorz, I. Komaromi, R. L. Martin, D.J. Fox, T. Keith, M. A. Al-Laham, C. Y. Peng, A. Nanayakkara, M. Challacombe, P. M. W. Gill, B. Johnson, W. Chen, M. W. Wong, C. Gonzalez, and J. A. Pople, Gaussian, Inc., Wallingford CT, 2004.

2. (a) Casida, M. E. In Recent Advances in Density Functional Methods, part I; Chong, D. P., Eds.; World Scientific: Singapore, 1995; pp 155-192. (b) Gross, E. K. U.; Dobson, J. F.; Petersilka, M. Top. Curr. Chem. 1996, 181, 81-172. (c) Gross, E. K. U.; Kohn, W. Adv. Quantum Chem. 1990, 21, 255-291. (d) Runge, E.; Gross, E. K. U. Phys. Rev. Lett. 1984, 52, 997-1000.

3. (a) Miertus, S.; Tomasi, J. Chem. Phys. 1982, 65, 239-245. (b) Tomasi, J.; Persico, M. Chem. Rev. 1994, 94, 2027-2094. (c) Cammi, R.; Tomasi, J. J.Comp.Chem. 1995, 16, 1449-1458. 
Table S1 B3LYP/6-31+G (d, p) optimized lowest energy 3D conformers of compounds $\mathbf{1}, \mathbf{3}$, and $\mathbf{4}$ and their boltzmann distributions.

\begin{tabular}{|c|c|c|}
\hline NO. & $\begin{array}{l}\text { Boltzmann } \\
\text { Distribution }\end{array}$ & Lowest energy 3D conformers \\
\hline 1-1 & 62.1 & \\
\hline $1-2$ & 19.2 & \\
\hline $1-3$ & 17.9 & \\
\hline $1-4$ & 0.4 & \\
\hline $1-5$ & 0.4 & \\
\hline
\end{tabular}




\begin{tabular}{|c|c|c|}
\hline NO. & $\begin{array}{l}\text { Boltzmann } \\
\text { Distribution }\end{array}$ & Lowest energy $3 \mathrm{D}$ conformers \\
\hline 3-1 & 37.2 & \\
\hline $3-2$ & 36.1 & \\
\hline 3-3 & 21.3 & \\
\hline $3-4$ & 5.3 & \\
\hline $3-5$ & 0.1 & \\
\hline
\end{tabular}




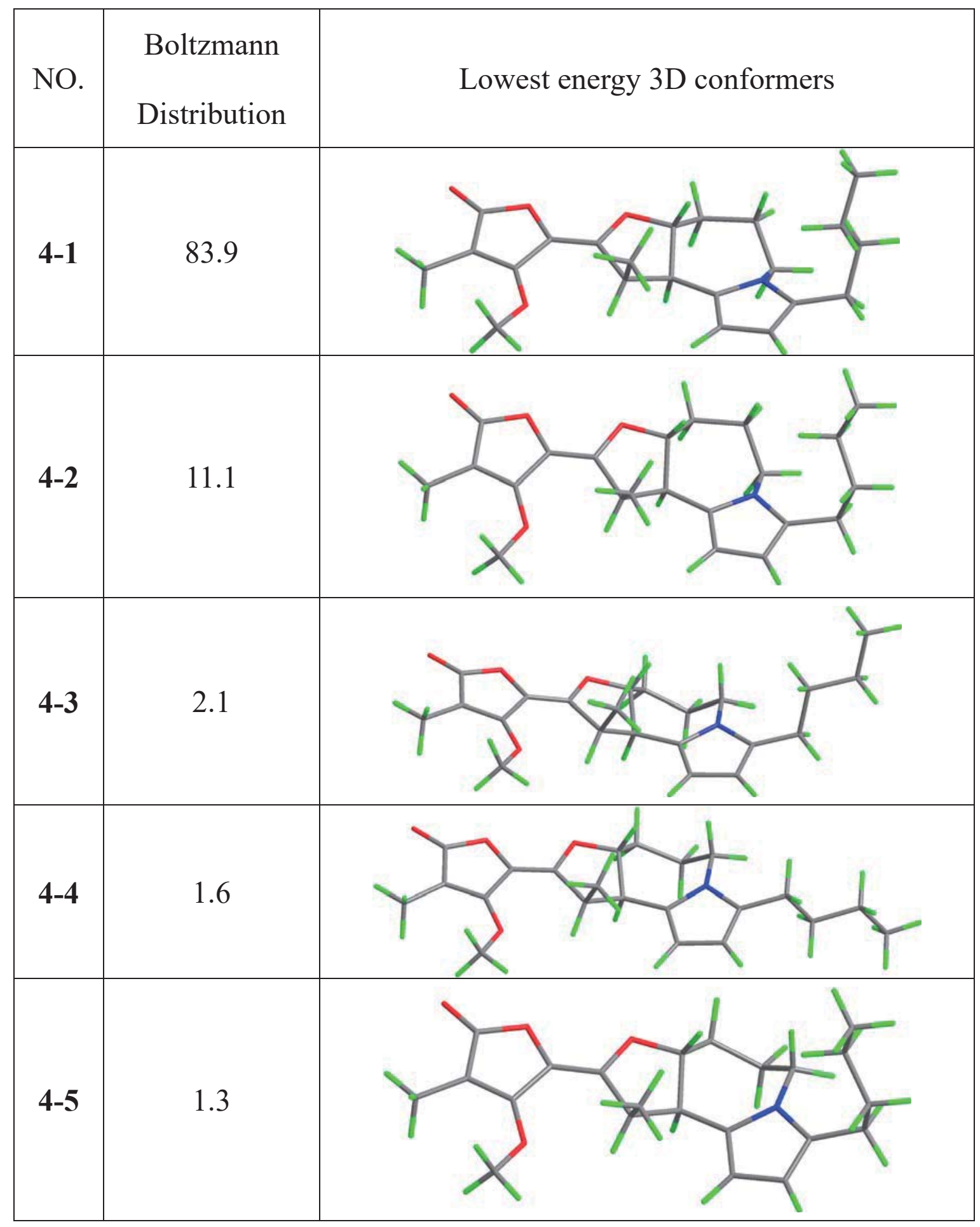




\section{HPLC Map}

HPLC Map was performed on an Agilent 1260 liquid chromatograph with a Cosmosil $\mathrm{C}-18,5 \phi 5 \mu \mathrm{m}, 4.5 \mathrm{~mm} \times 250 \mathrm{~mm}$, column [mobile phase $\mathrm{MeOH} / \mathrm{H}_{2} \mathrm{O}, 1: 9-0: 1, \mathrm{v} / \mathrm{v}, 5$ $\mu \mathrm{L}$ (5.5 mg alkaloid extraction in $1 \mathrm{~mL}), 60 \mathrm{~min}, 1.0 \mathrm{~mL} / \mathrm{min}]$, UV detected in 290 nm.Compound 4 (10-epi-3-n-butylneostemonine), 10 (protostemonine), 12 (stemofoline), protostemotinine, oxymaistemonine, and isostemonamide were assigned.

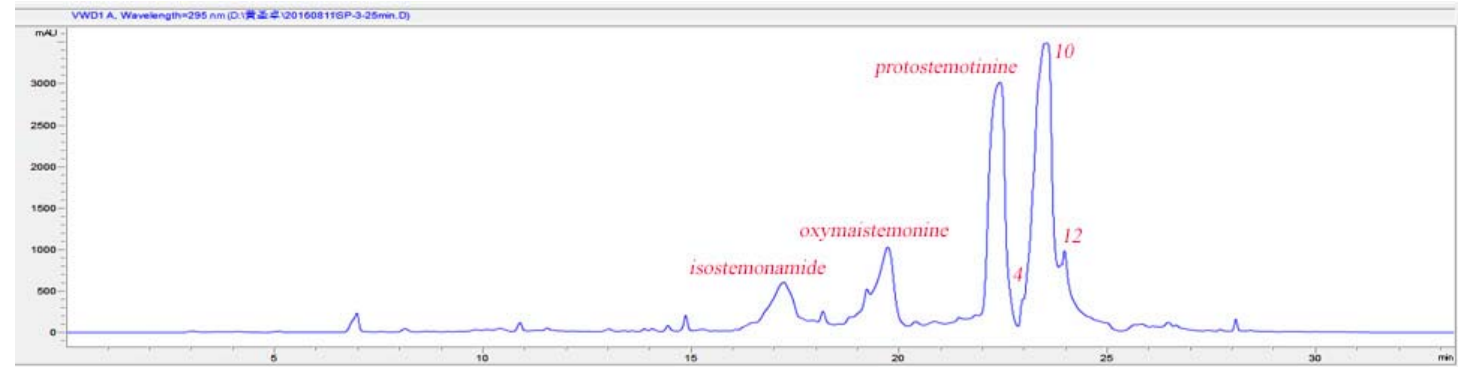

Table S2 Nematicidal activity with RDR of isolated compounds

\begin{tabular}{llll}
\hline Compound & RDR (\%) & Compound & RDR (\%) \\
\hline $\mathbf{1}$ & $45.82 \pm 0.58$ & Protostemonine & $11.40 \pm 1.80$ \\
$\mathbf{2}$ & $29.70 \pm 0.39$ & Isostemonamide & $22.61 \pm 2.03$ \\
$\mathbf{3}$ & $7.20 \pm 2.89$ & Stemonamide & $14.51 \pm 1.87$ \\
$\mathbf{4}$ & $16.49 \pm 1.18$ & Isooxymaistemonine & $30.59 \pm 1.65$ \\
$\mathbf{5}$ & $37.55 \pm 7.79$ & Protostemonamide & $10.09 \pm 2.67$ \\
$\mathbf{6}$ & $19.87 \pm 2.25$ & $(2 ' S)$-Hydroxystemofoline & $7.862 \pm 6.17$ \\
$\mathbf{7}$ & $20.41 \pm 2.67$ & Stemofoline & $12.72 \pm 1.19$ \\
$\mathbf{8}$ & $21.51 \pm 3.83$ & Didehydroprotostemonine & $30.78 \pm 11.27$ \\
$\mathbf{9}$ & $51.44 \pm 5.39$ & Oxymaistemonine & $19.13 \pm 1.06$ \\
$\mathbf{1 0}$ & $93.94 \pm 2.78$ & (-)-Stemonine & $12.53 \pm 3.70$ \\
$\mathbf{1 1}$ & $45.56 \pm 3.13$ & $(12 R)$-Dihydroprotostemonine & $15.22 \pm 3.35$ \\
$\mathbf{1 2}$ & $98.28 \pm 1.45$ & (+)-Oxystemofoline & $28.87 \pm 3.63$ \\
Protostemotinine & $10.17 \pm 4.01$ & Albendazole & $53.47 \pm 3.46$ \\
\hline
\end{tabular}


Figure S2. The Key HMBC $(\rightarrow)$ and ${ }^{1} \mathrm{H}^{1} \mathrm{H}$ COSY $(-)$ correlations of 1-8

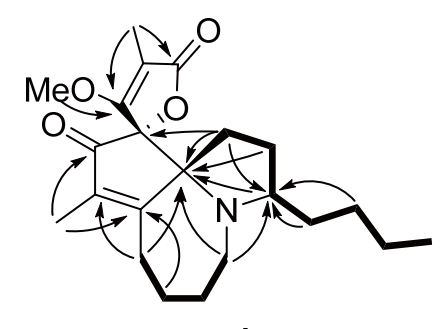

1
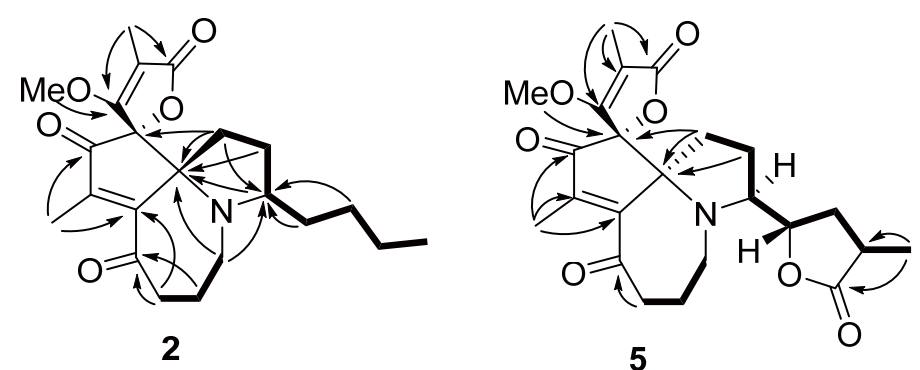

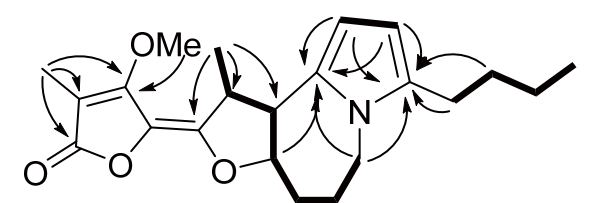

3,4

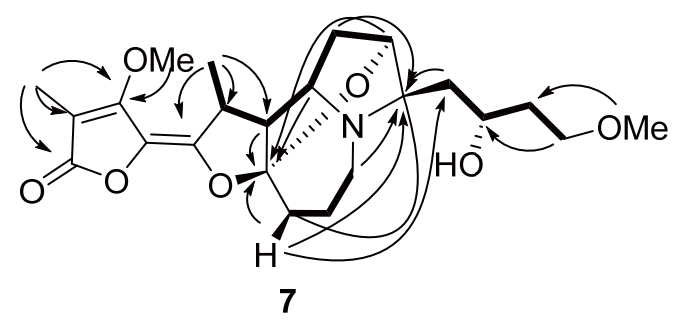

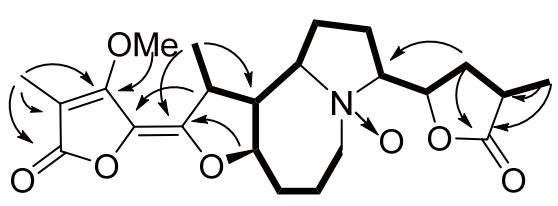

6

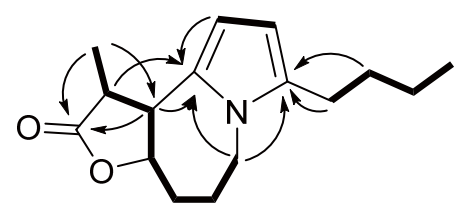

8 
Figure S3. The Key ROESY $(\leftrightarrow)$ correlations of 1-8
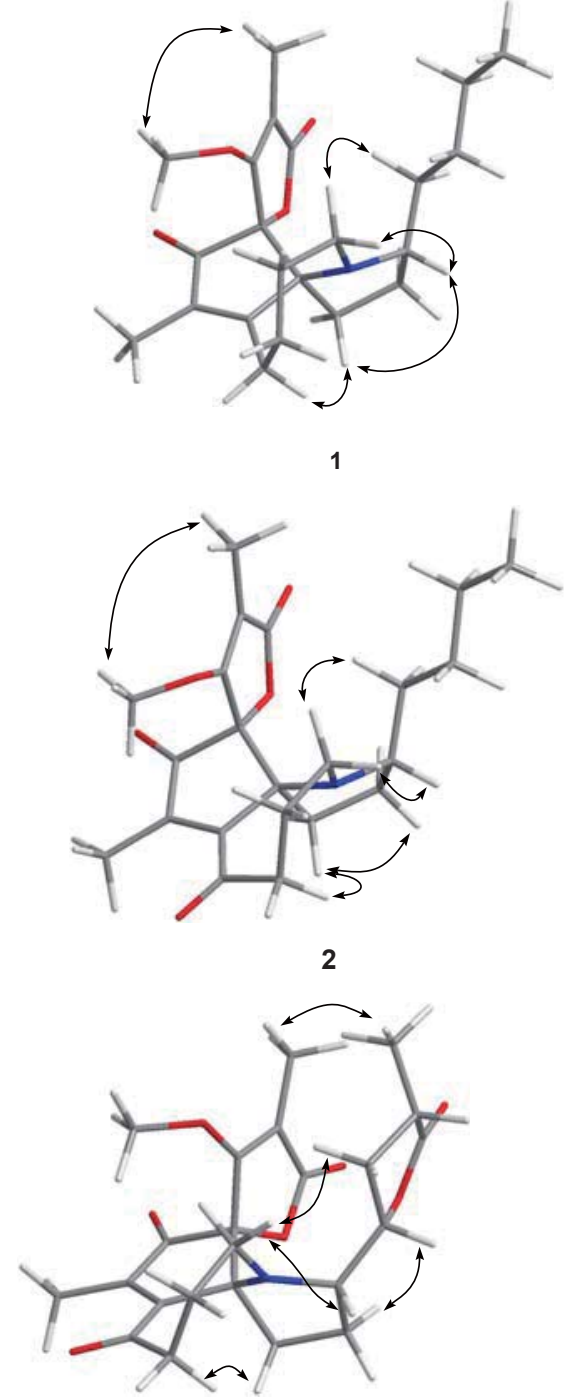

5

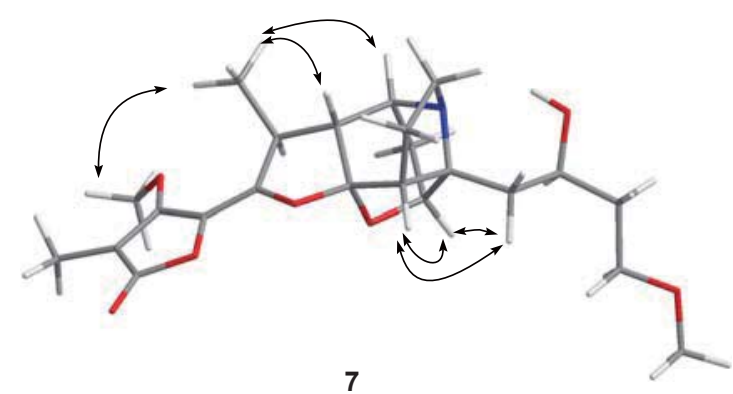

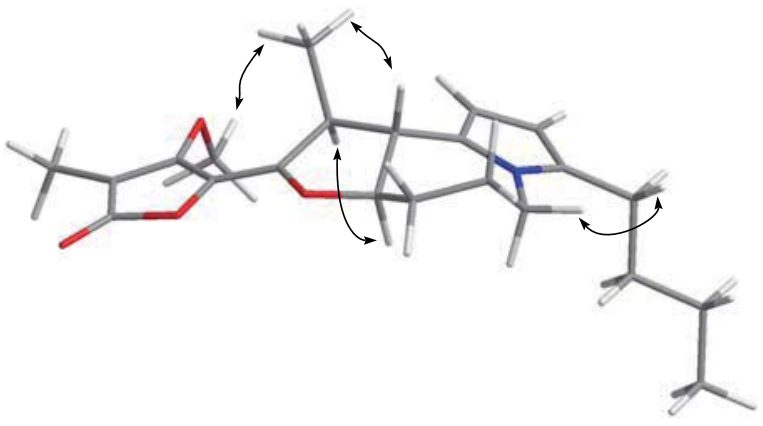

3

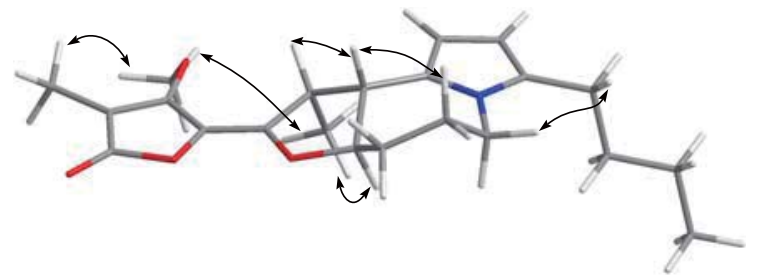

4

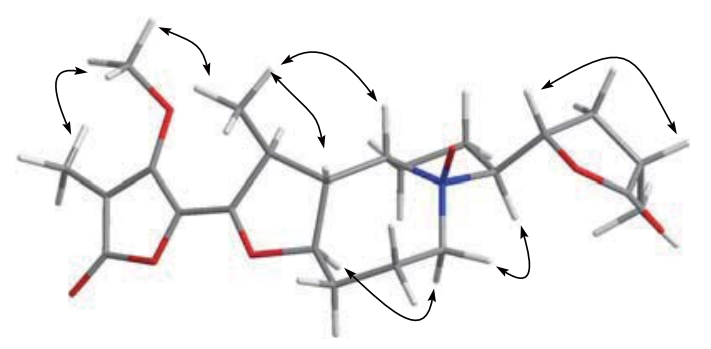

6

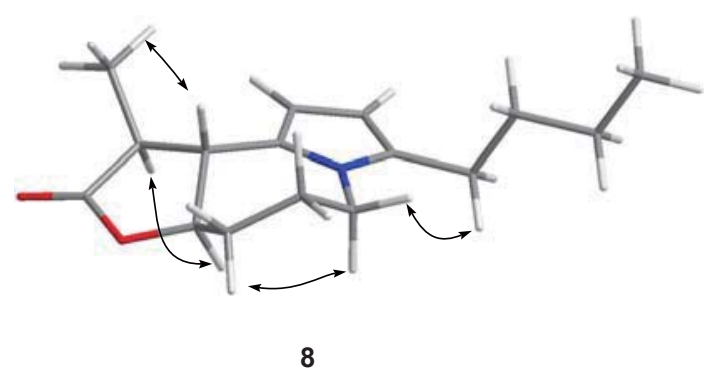


S3: ${ }^{1} \mathrm{H}$ NMR of $3 \beta$-n-butylstemonamine (1)

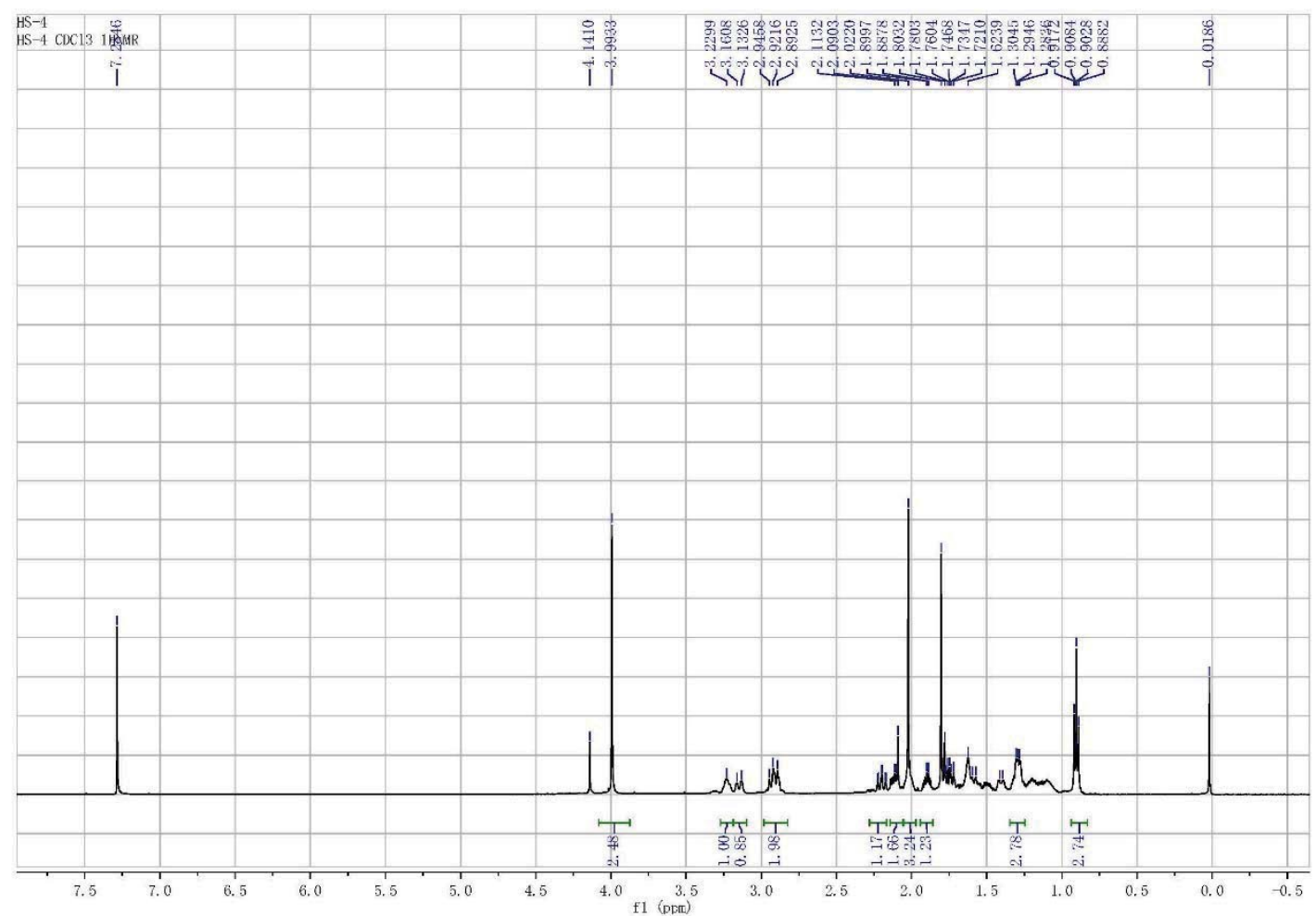

S4: DEPT of $3 \beta$-n-butylstemonamine (1)

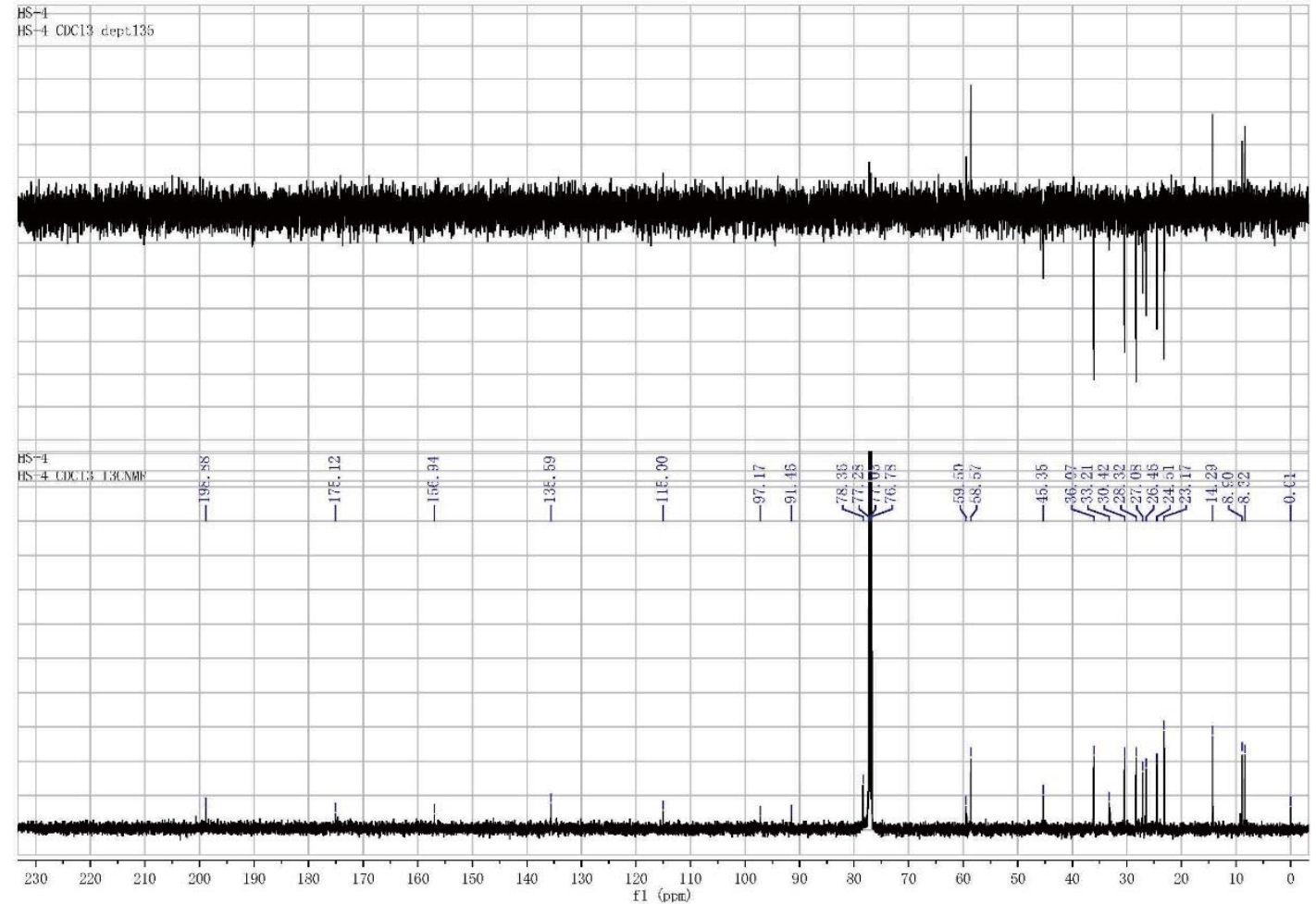


S5: HSQC of $3 \beta$-n-butylstemonamine (1)

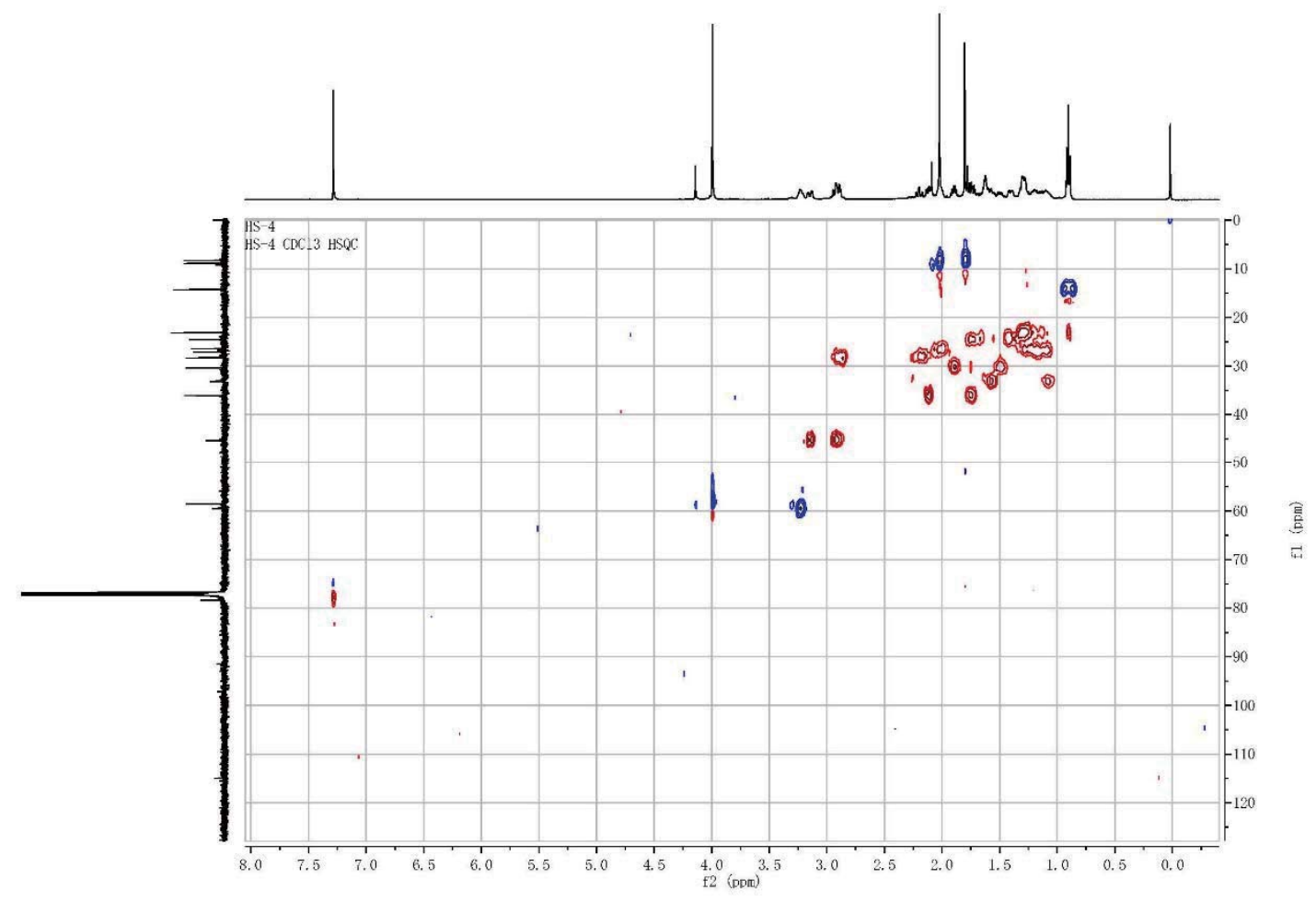

S6: HMBC of $3 \beta$ - $n$-butylstemonamine (1)

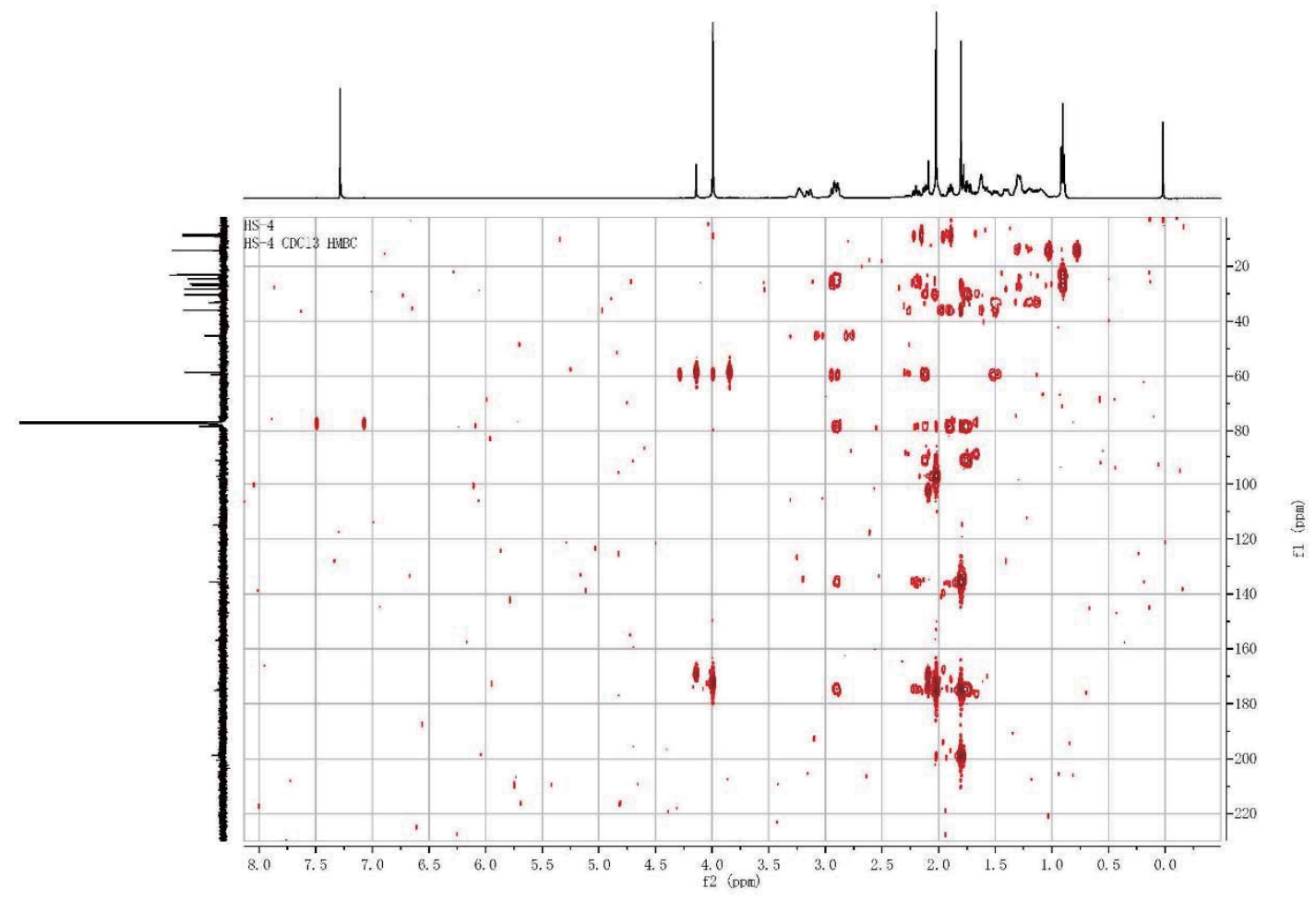


S7: ${ }^{1} \mathrm{H}^{1} \mathrm{H}$ COSY of $3 \beta$-n-butylstemonamine (1)

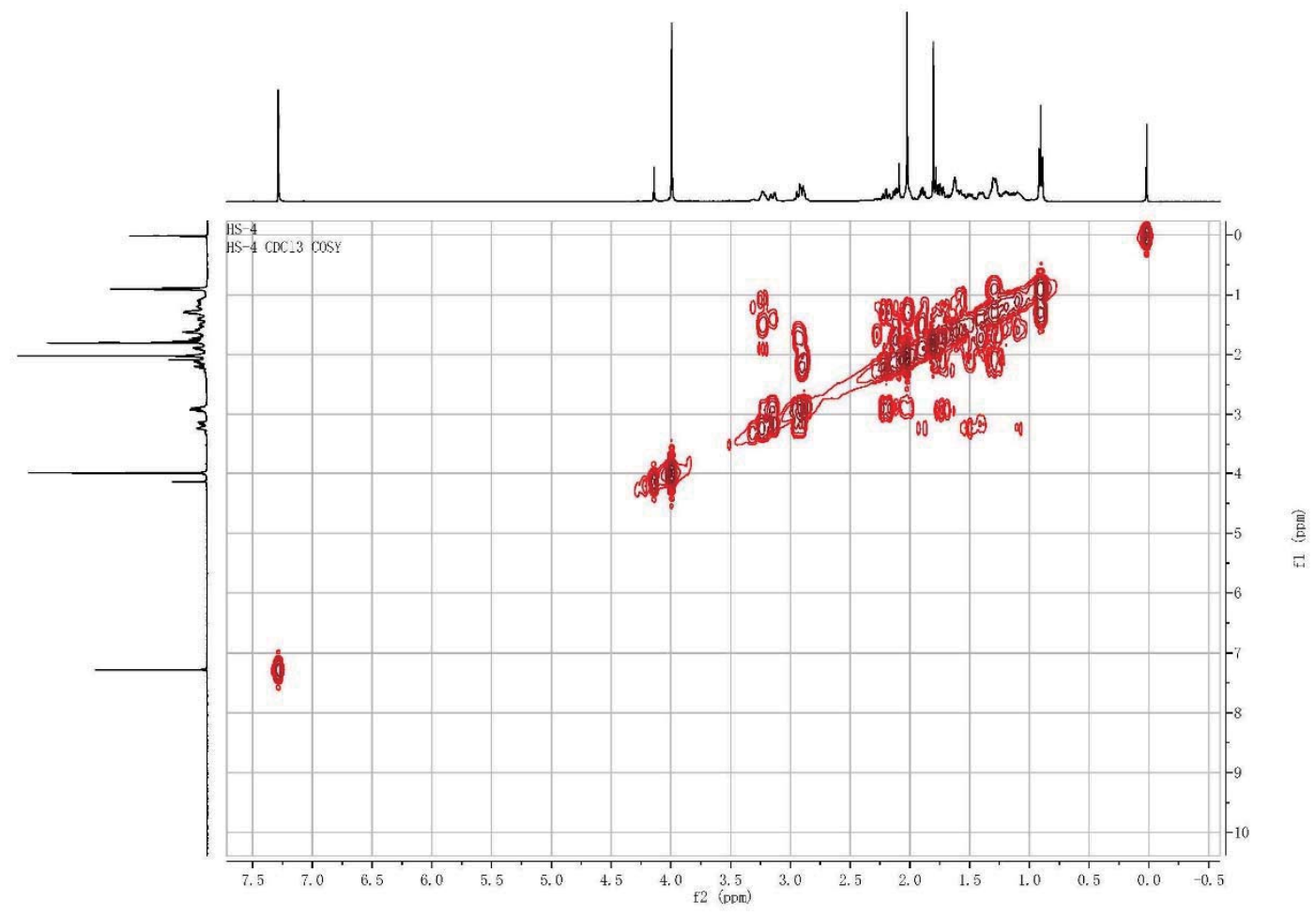

S8: ROESY of $3 \beta$-n-butylstemonamine (1)

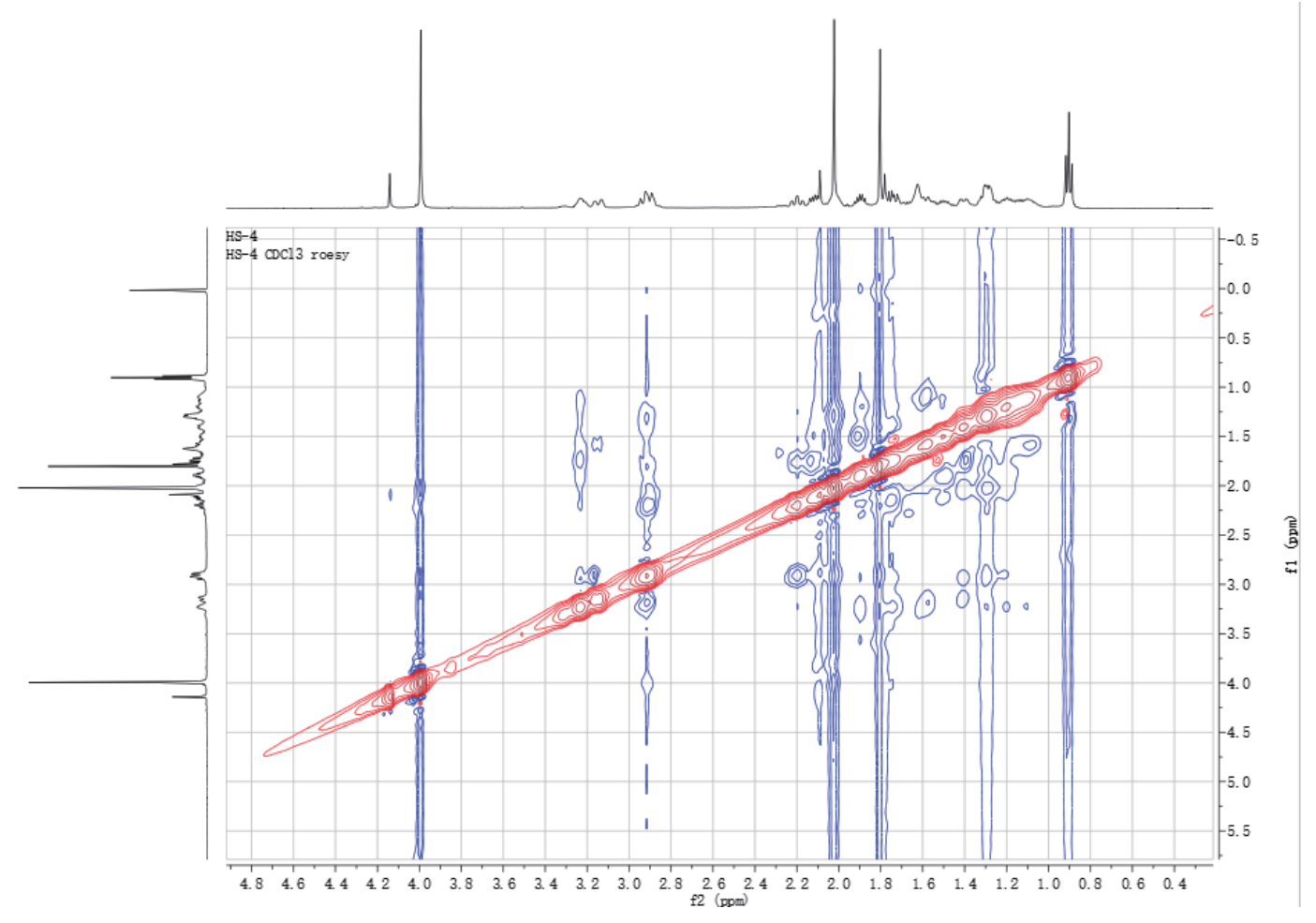


S9: ESIMS of $3 \beta$ - $n$-butylstemonamine (1)

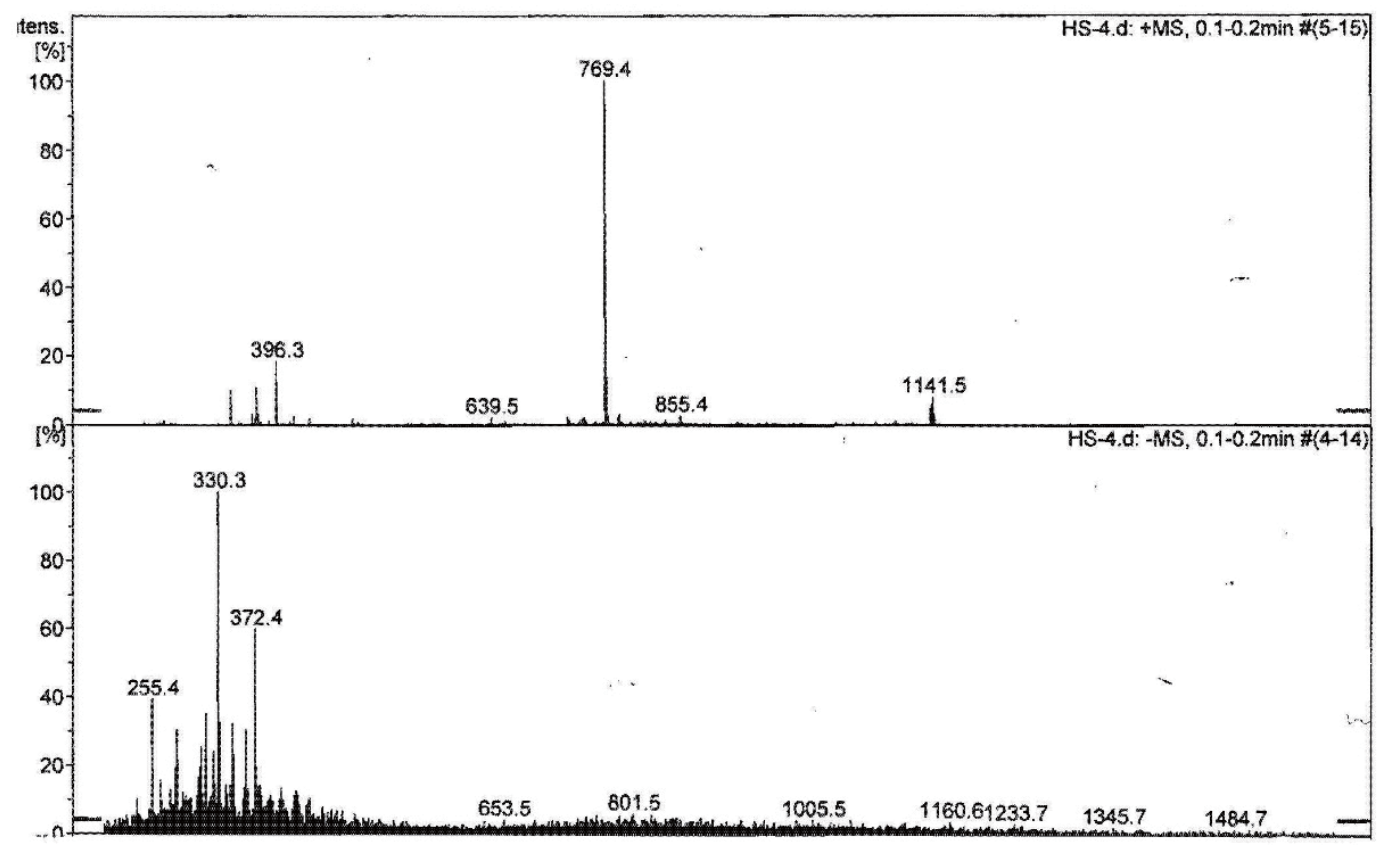


S10: ${ }^{1} \mathrm{H}$ NMR of 8-oxo-3 $\beta$-n-butylstemonamine (2)

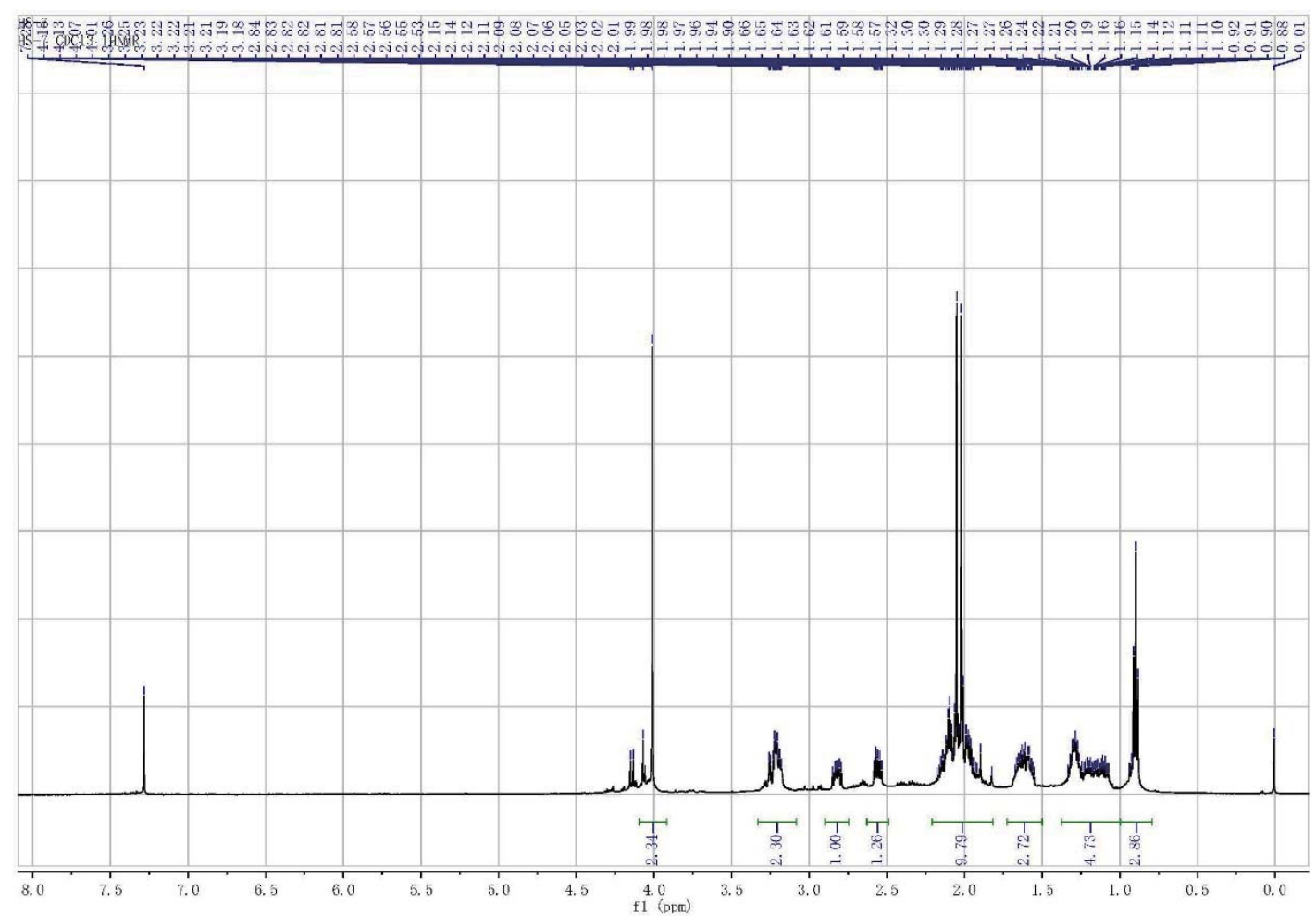

S11: DEPT of 8-oxo-3 $\beta$ - $n$-butylstemonamine (2)

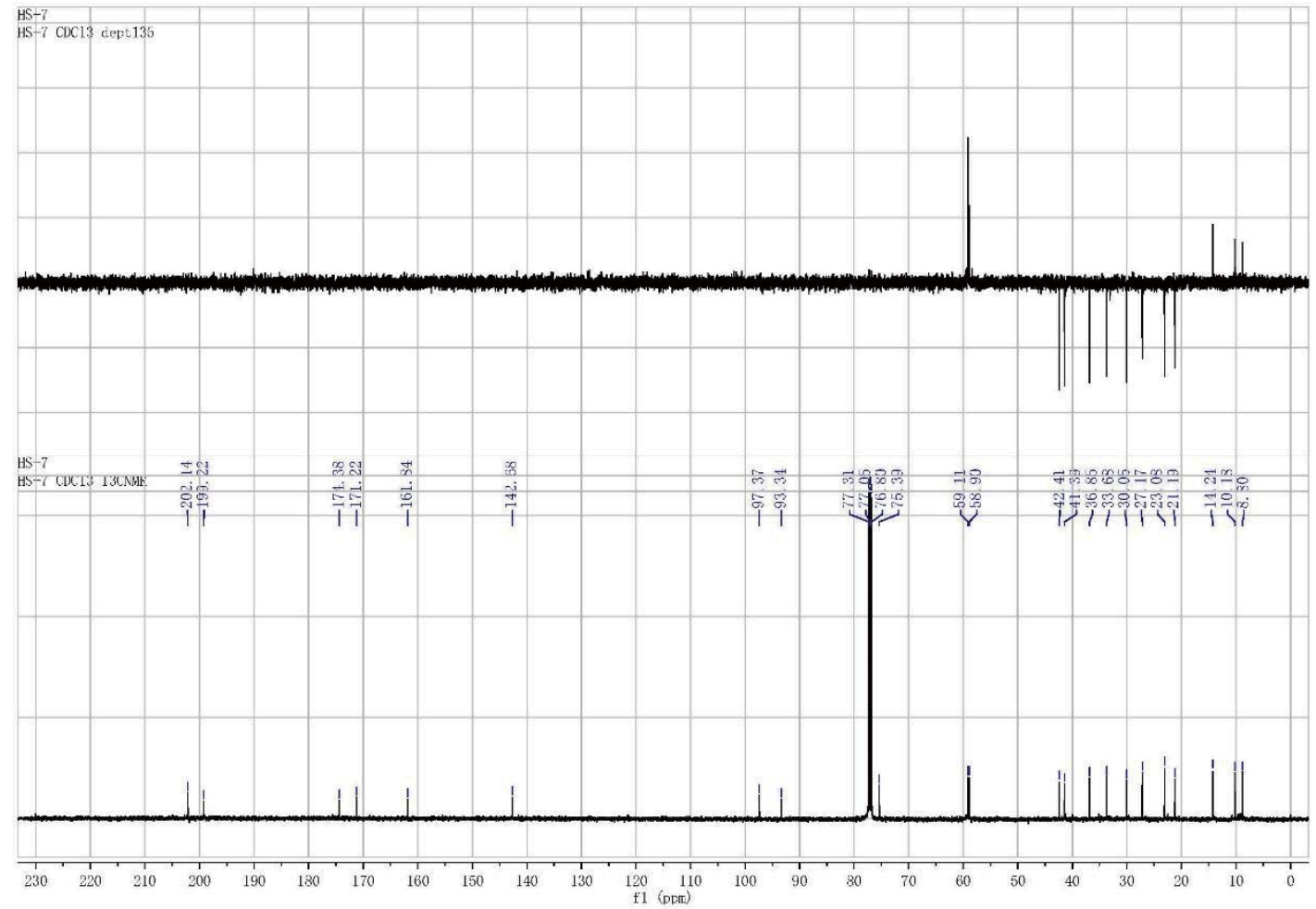


S12: HSQC of 8-oxo-3 $\beta$ - $n$-butylstemonamine (2)

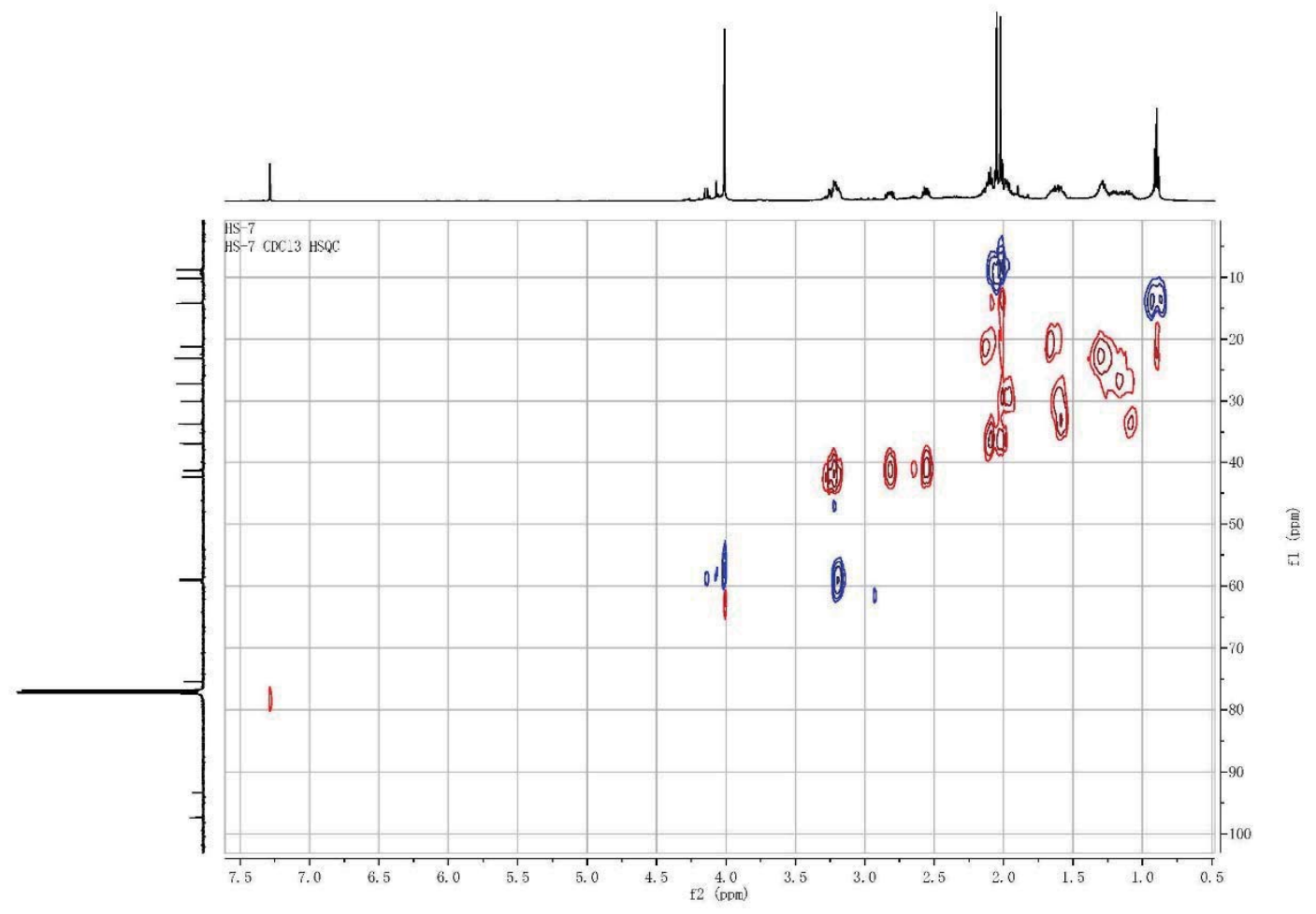

S13: HMBC of 8-oxo-3 $\beta$ - $n$-butylstemonamine (2)

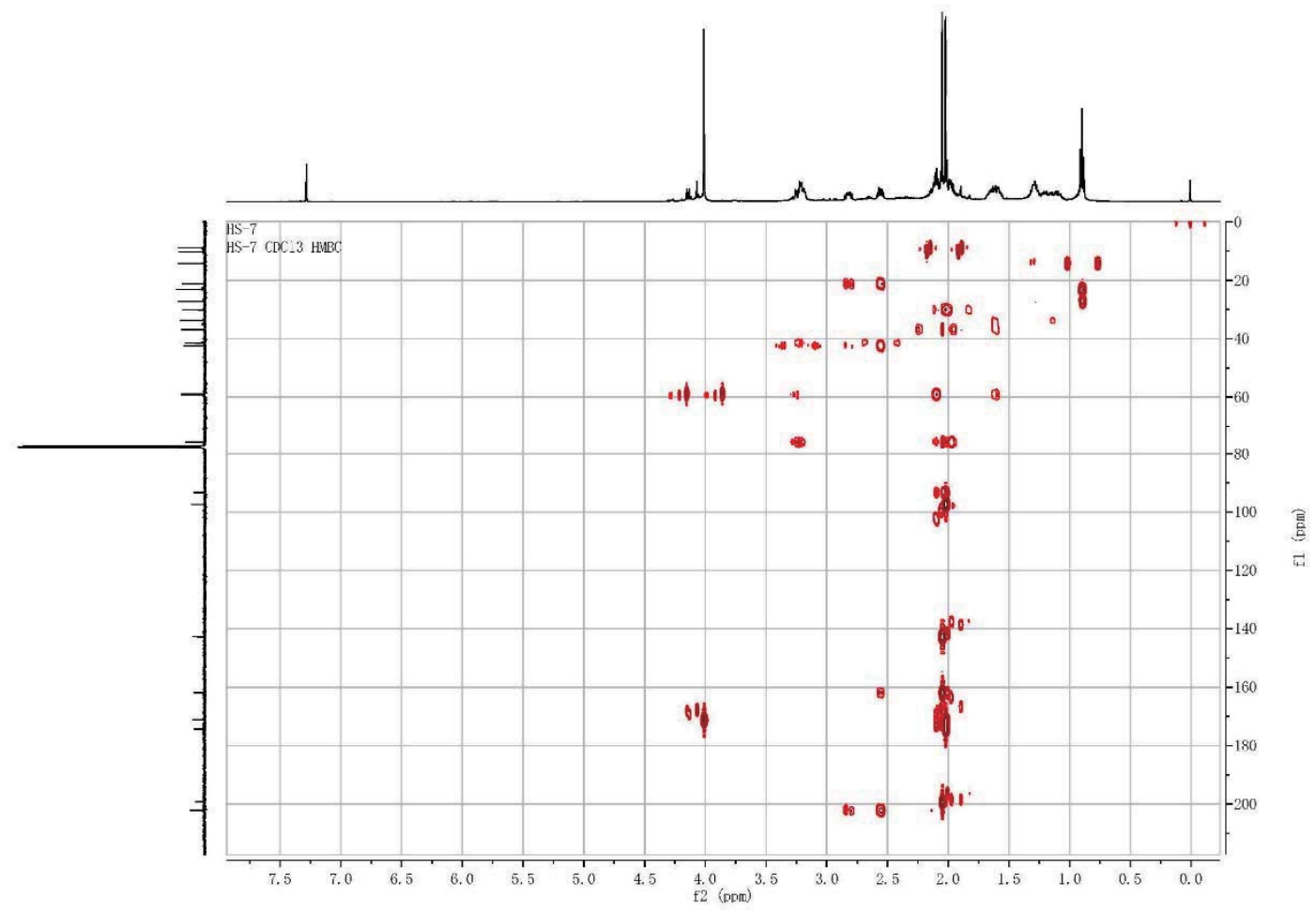


S14: ${ }^{1} \mathrm{H}{ }^{1} \mathrm{H}$ COSY of 8-oxo-3 $\beta$-n-butylstemonamine (2)

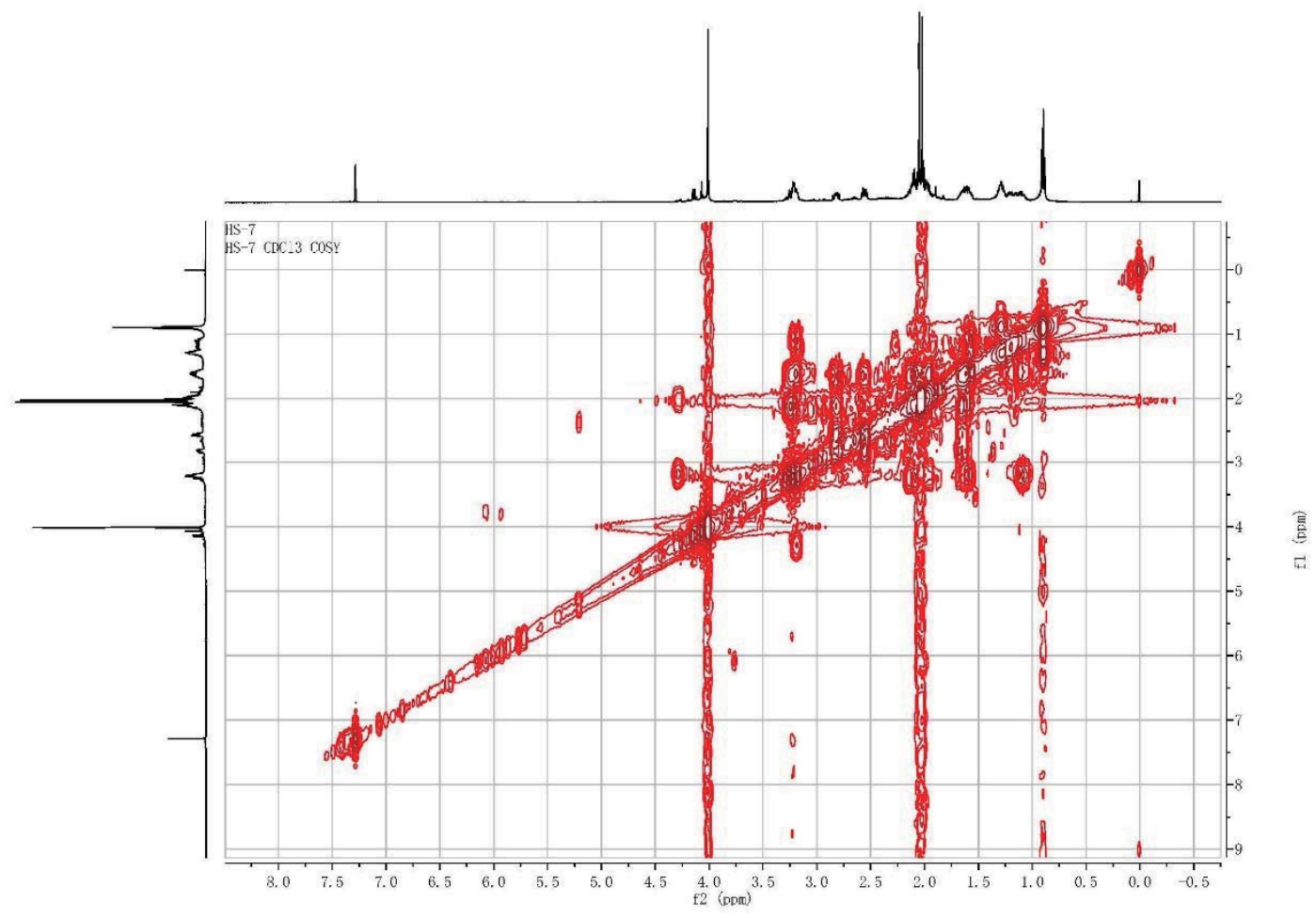

S15: ROESY of 8-oxo-3 $\beta$-n-butylstemonamine (2)

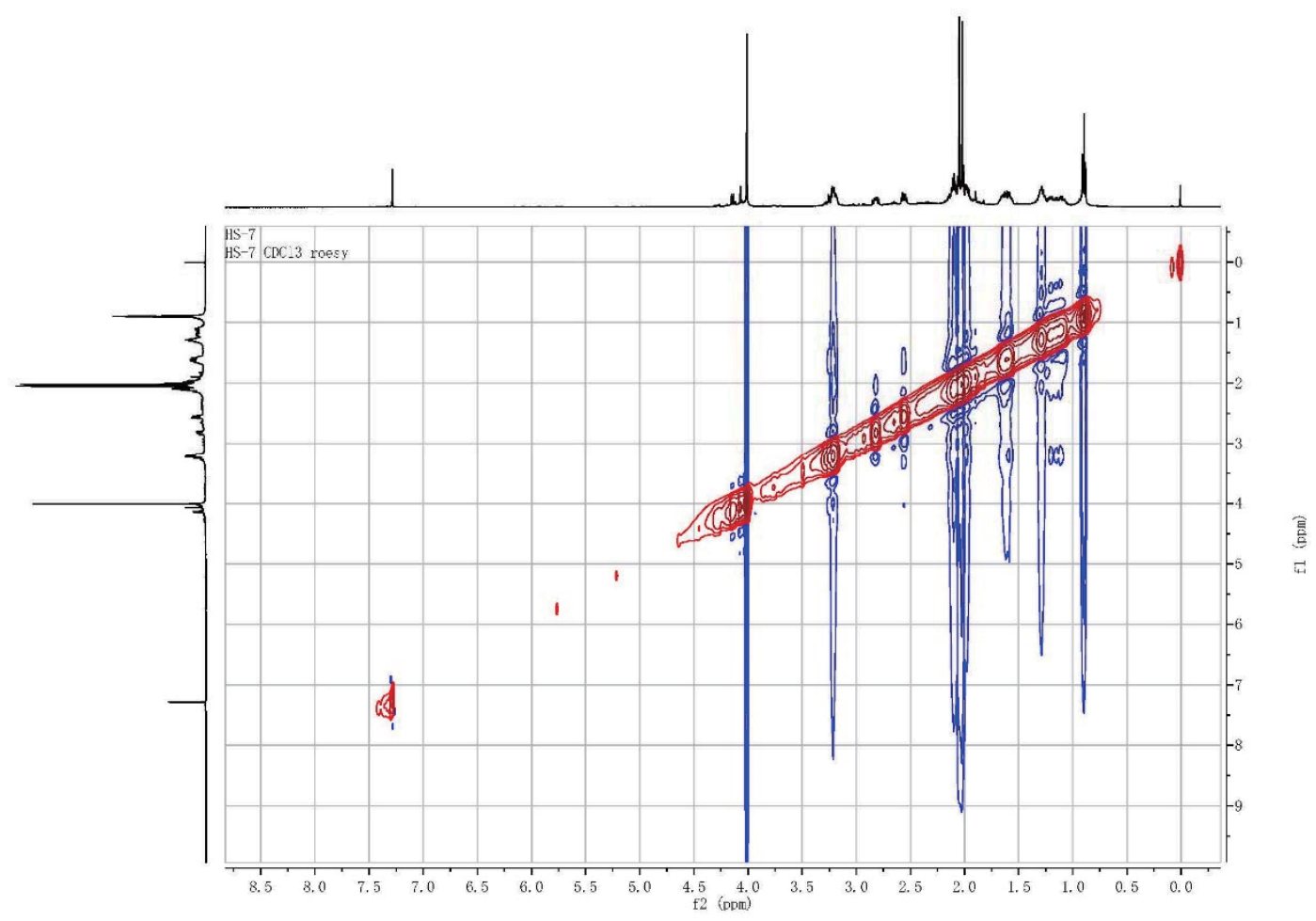


S16: ESIMS of 8-oxo-3 $\beta$ - $n$-butylstemonamine (2)

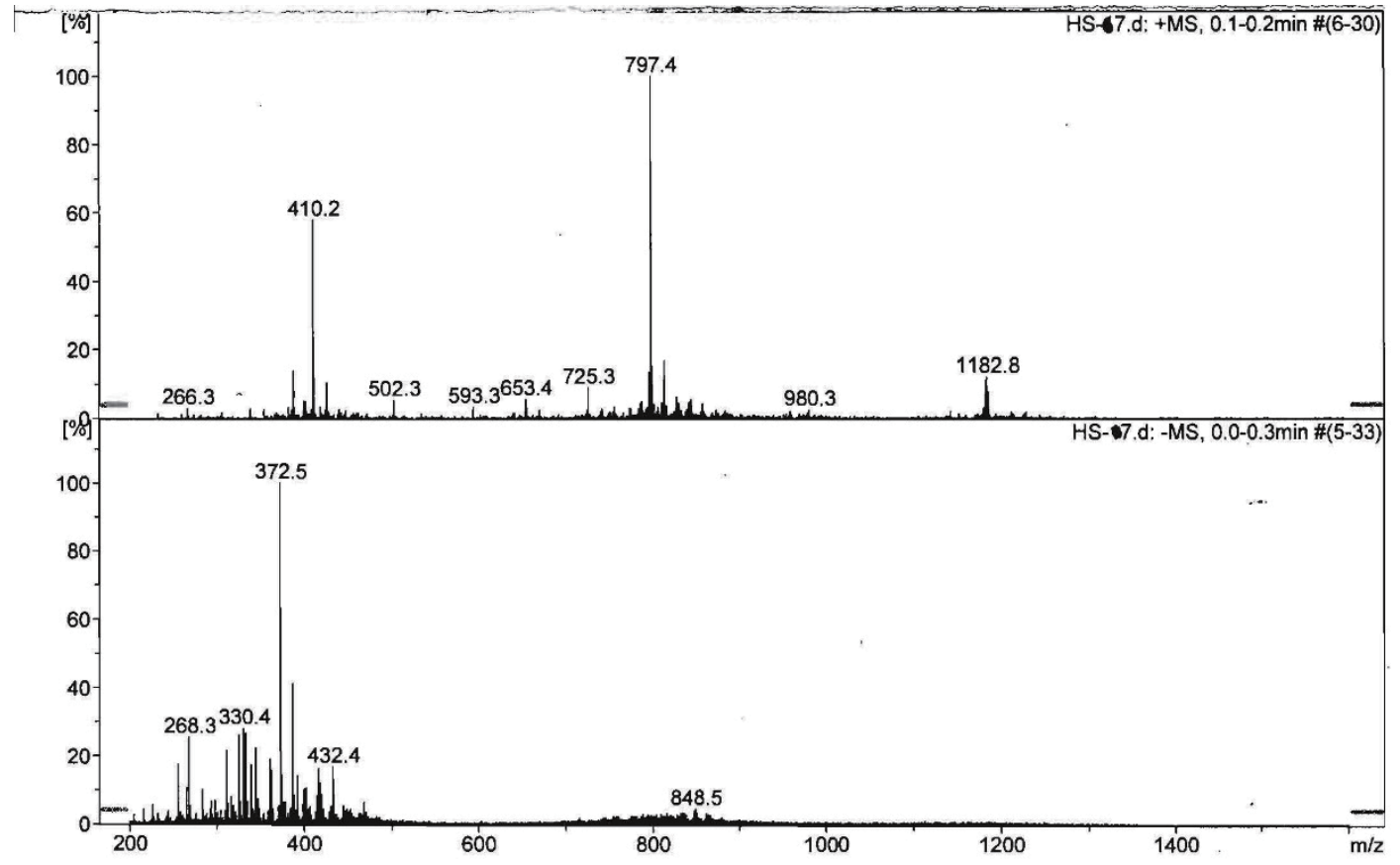


S17: 1H NMR of 3-n-butylneostemonine (3)

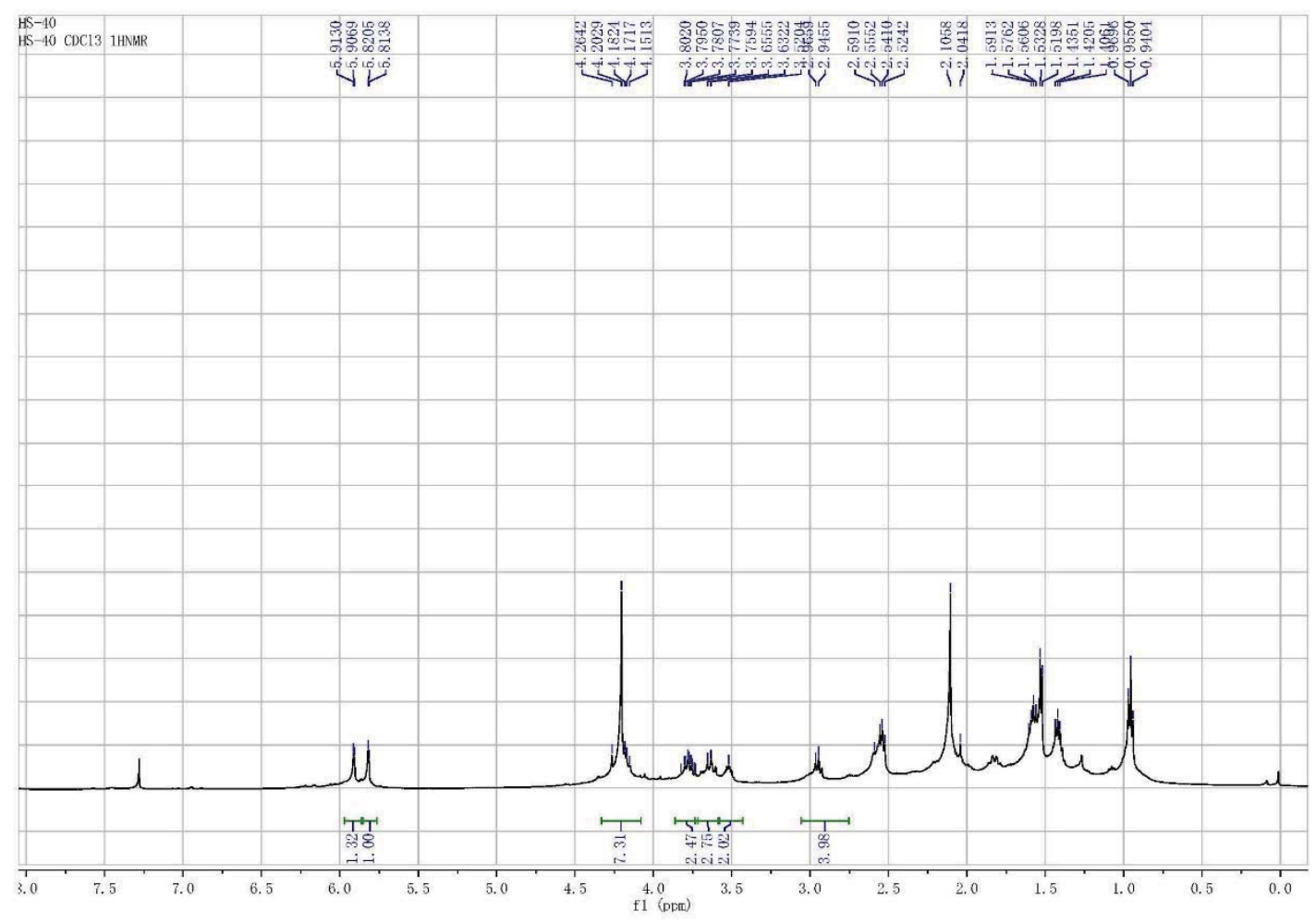

S18: DEPT of 3-n-butylneostemonine (3)

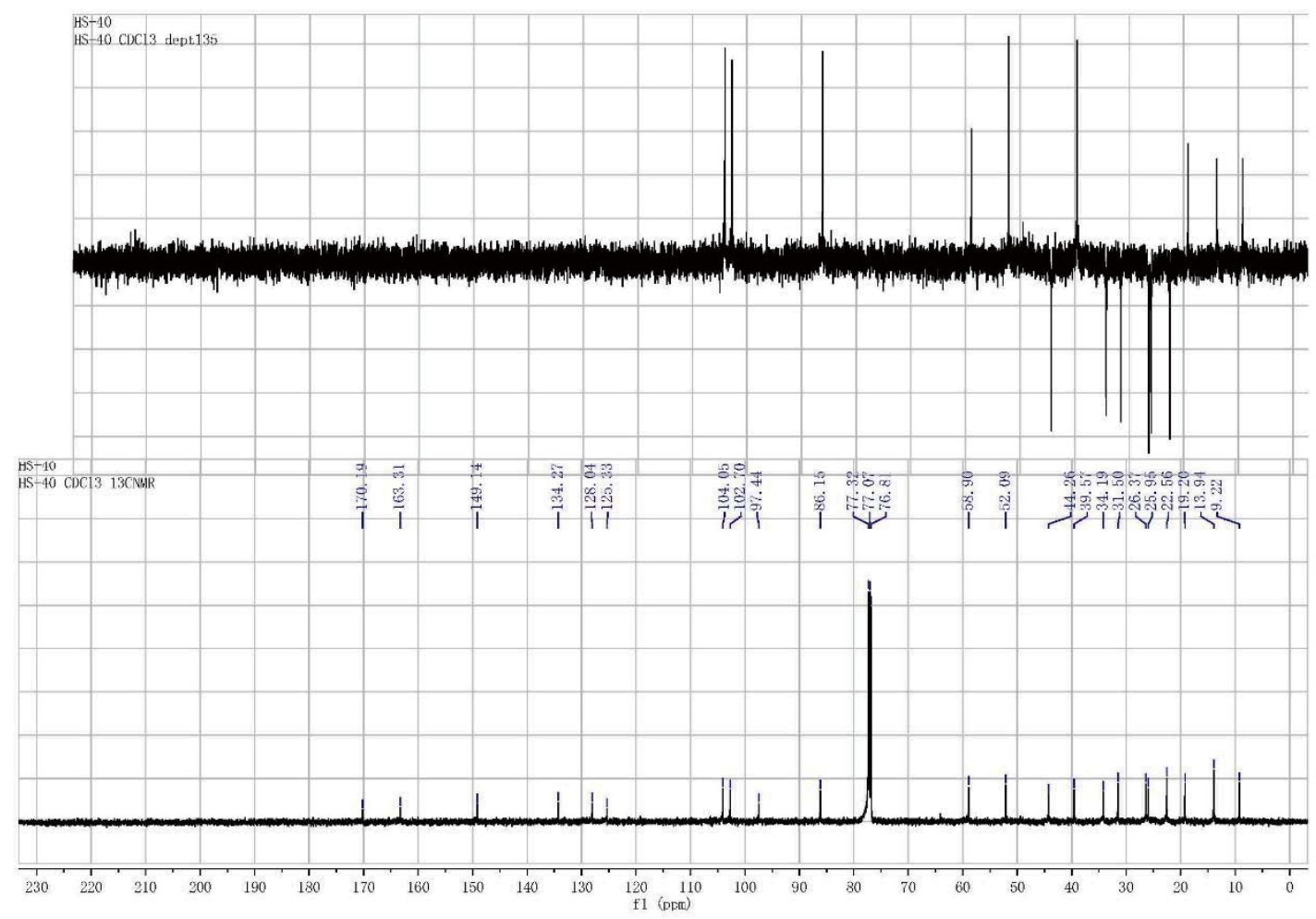


S19: HSQC of 3-n-butylneostemonine (3)

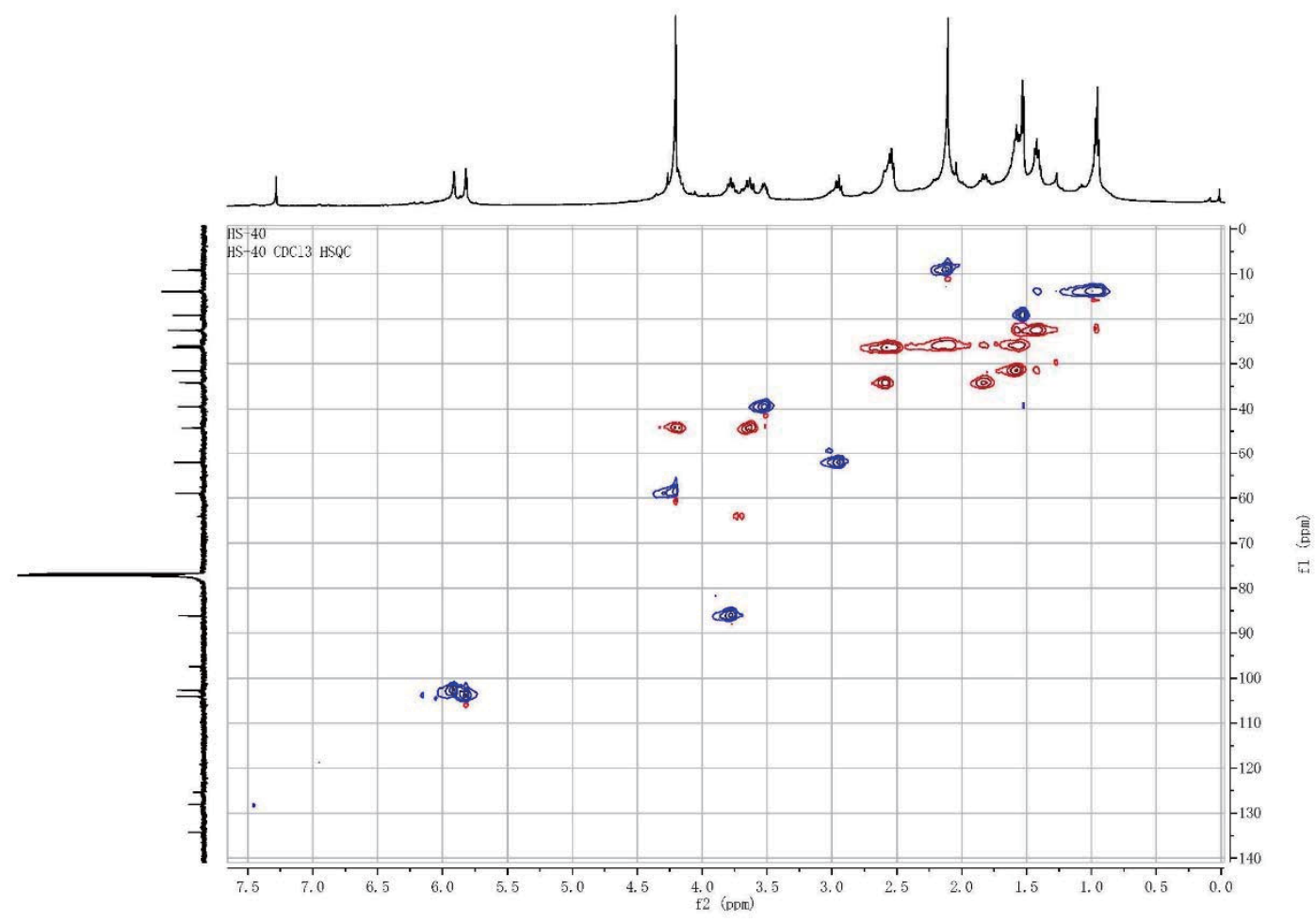

S20: HMBC of 3-n-butylneostemonine (3)

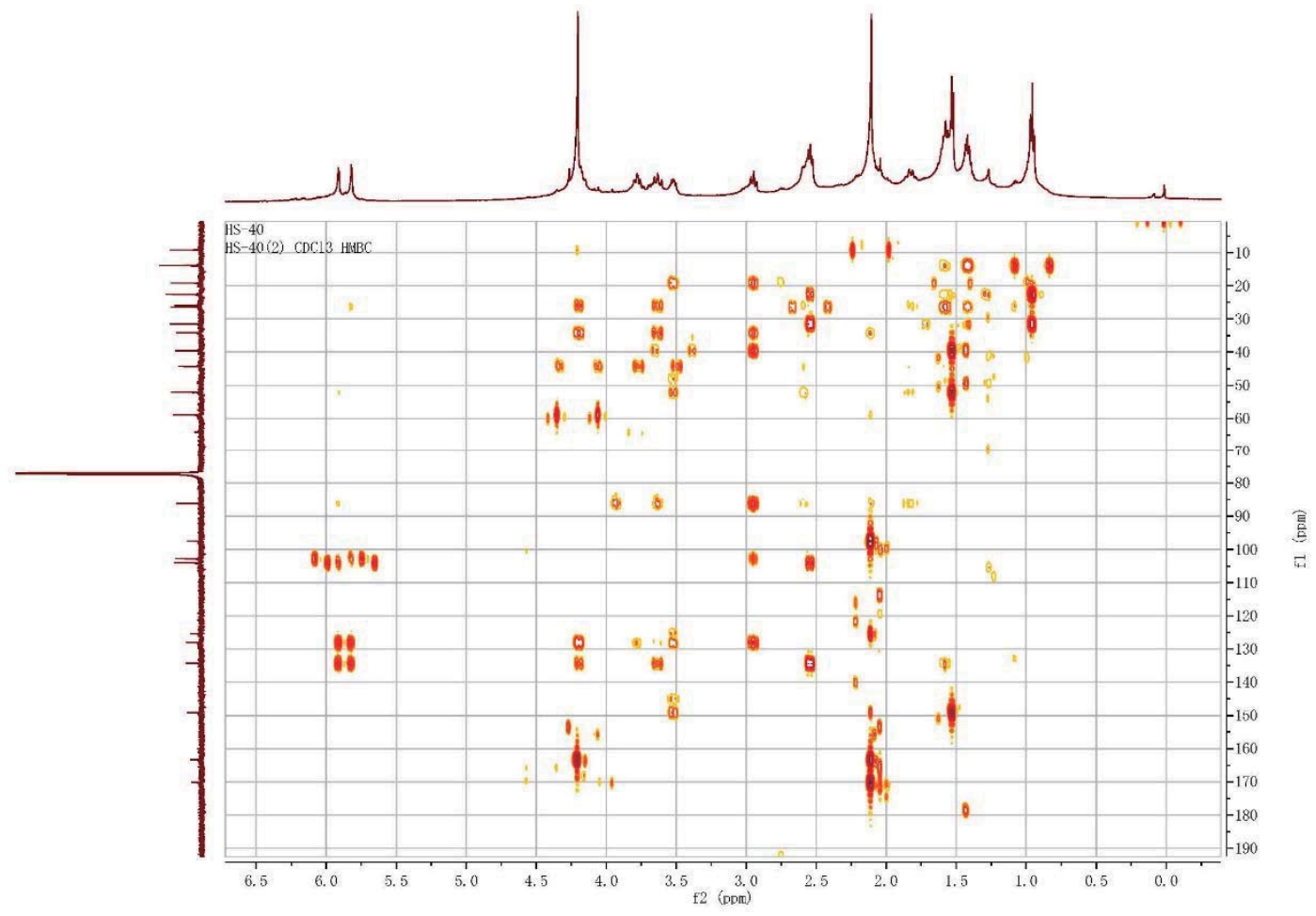


S21: ${ }^{1} \mathrm{H}{ }^{1} \mathrm{H}$ COSY of 3-n-butylneostemonine (3)

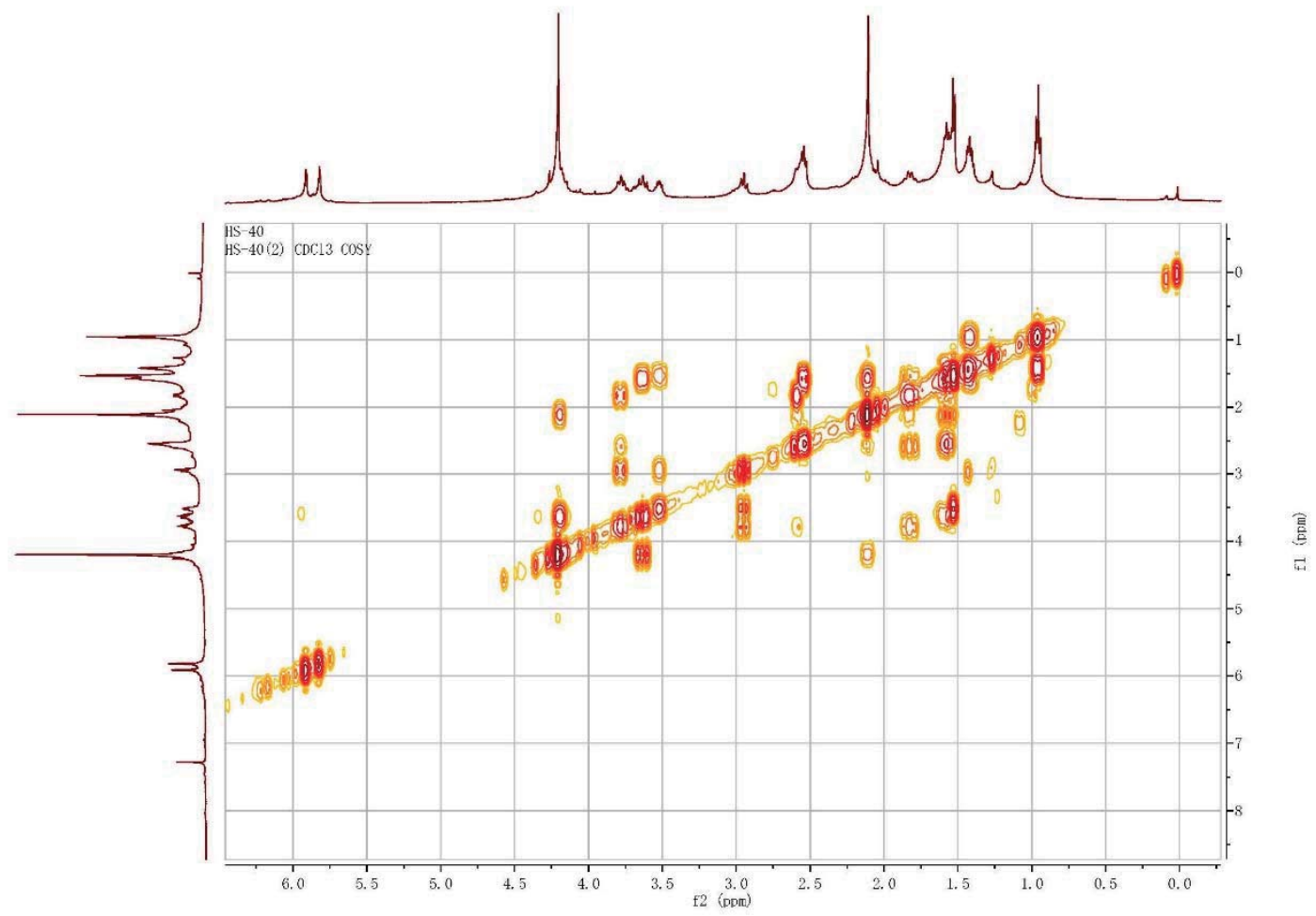

S22: ROESY of 3-n-butylneostemonine (3)

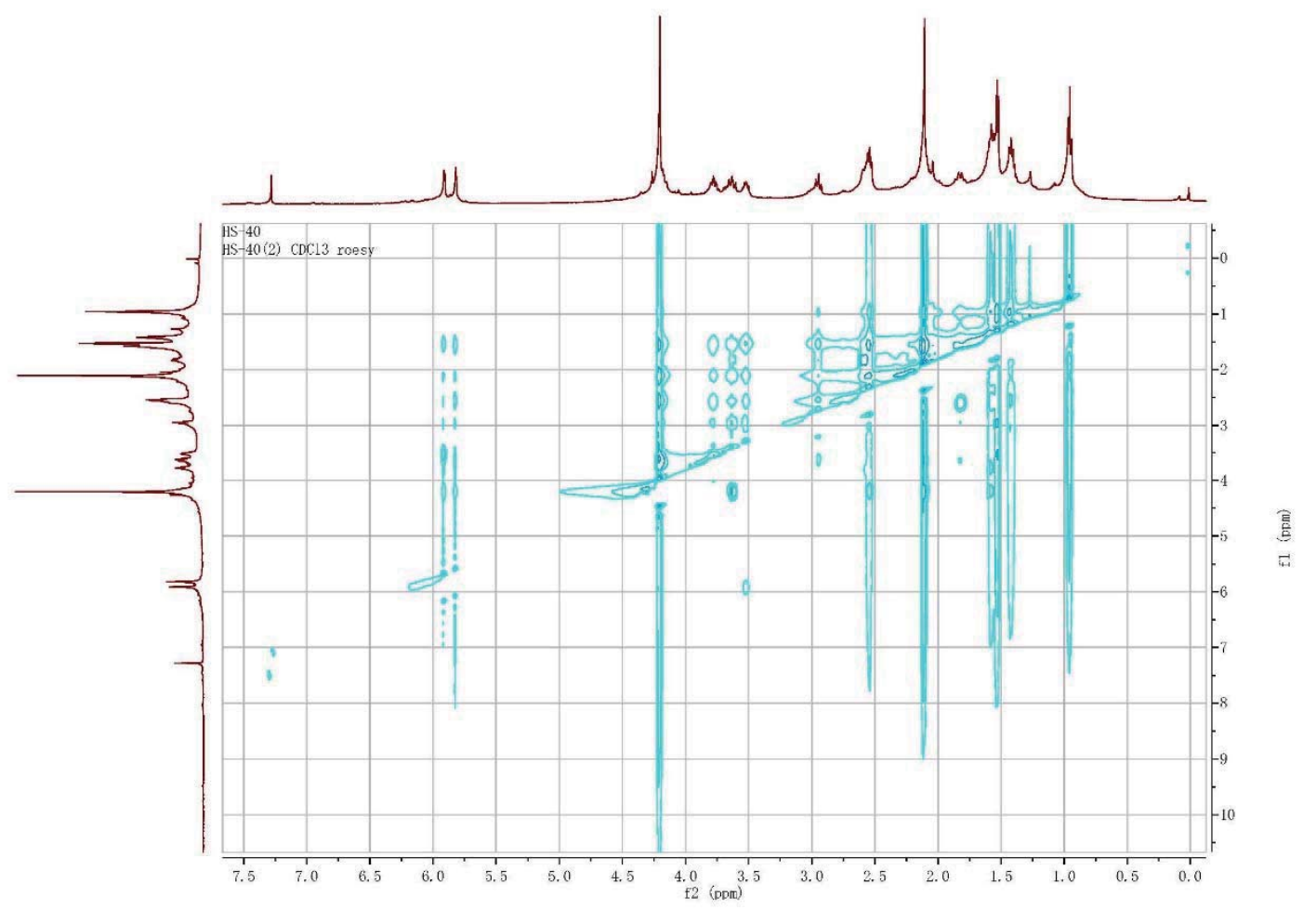


S23: EIMS of 3- $n$-butylneostemonine (3)

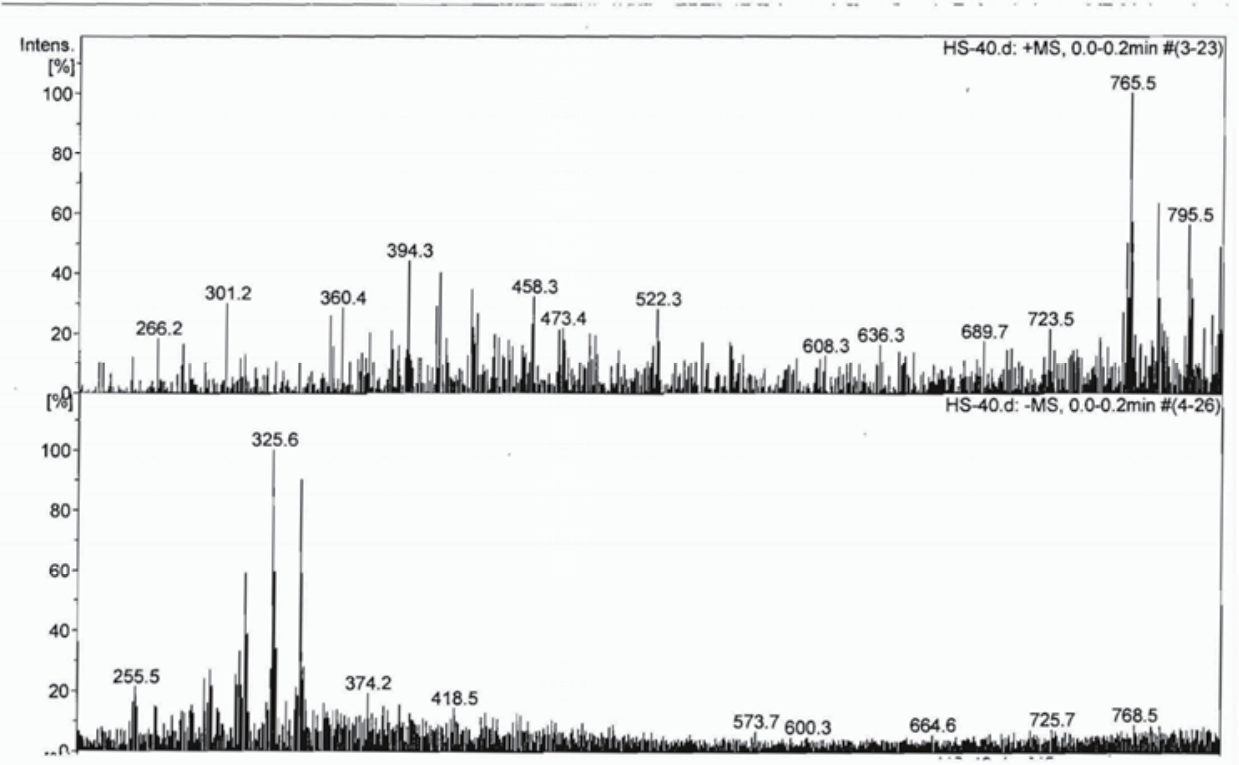


S24: ${ }^{1} \mathrm{H}$ NMR of 10-epi-3-n-butylneostemonine (4)

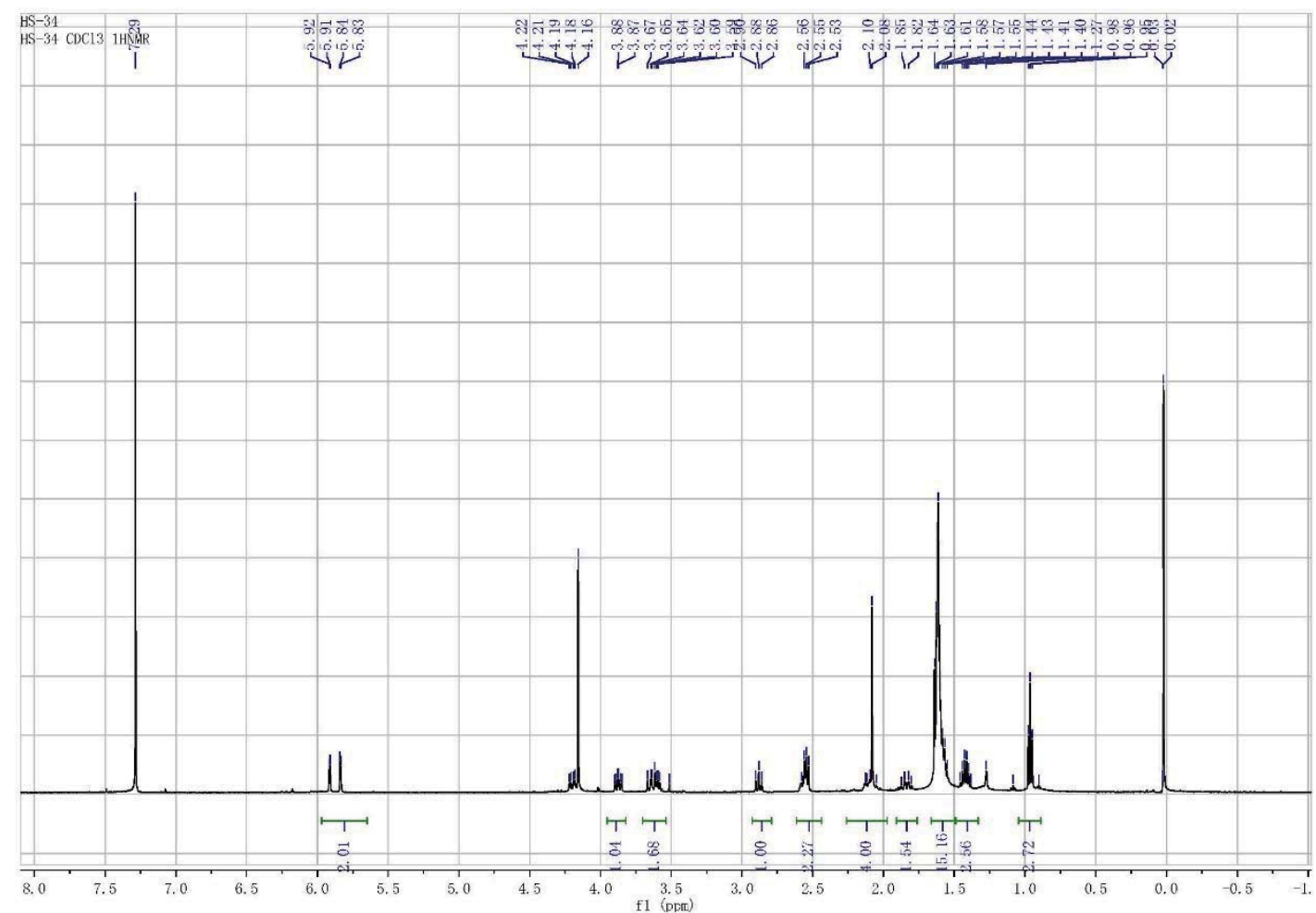

S25: DEPT of 10-epi-3-n-butylneostemonine (4)

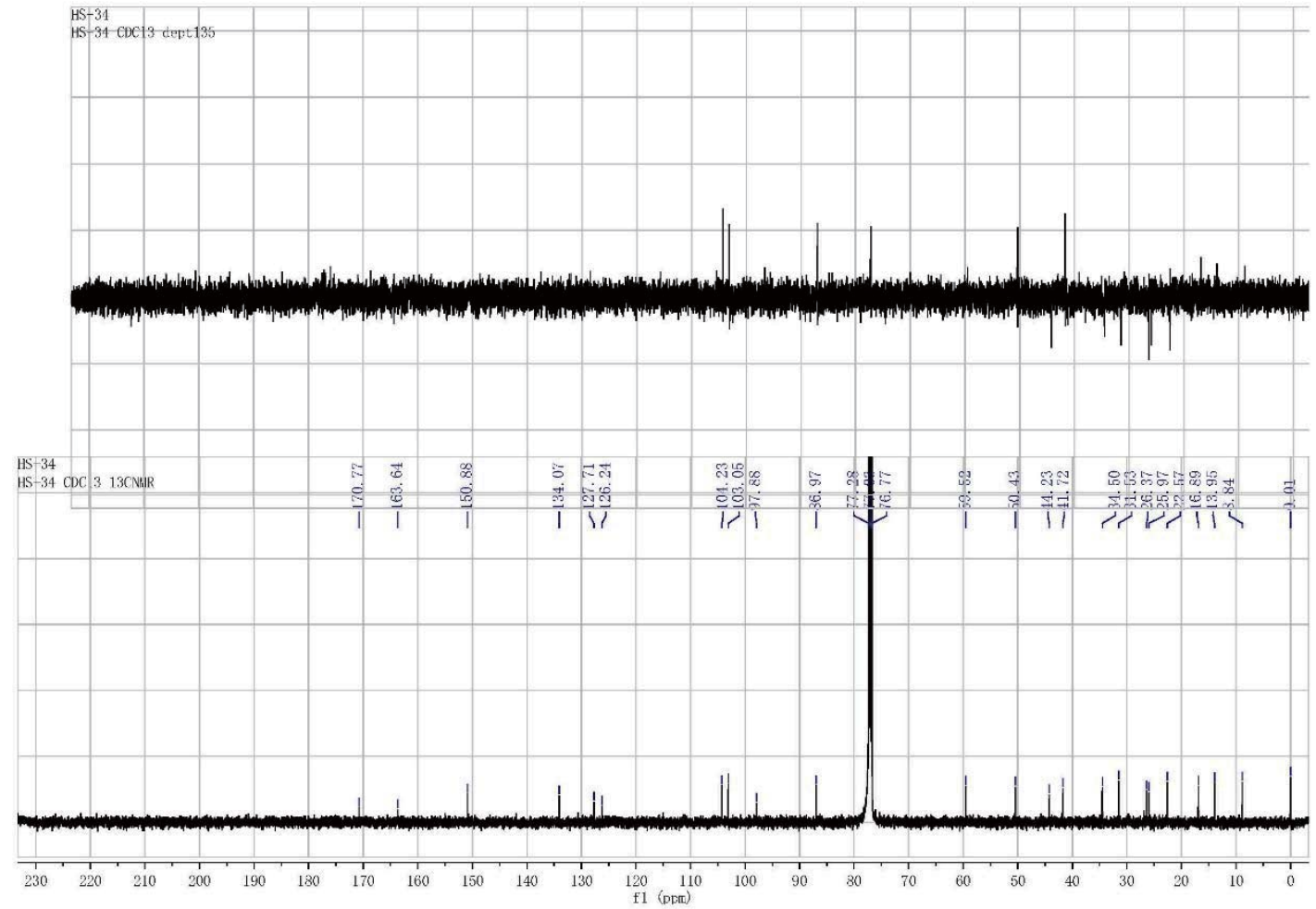


S26: HSQC of 10-epi-3-n-butylneostemonine (4)

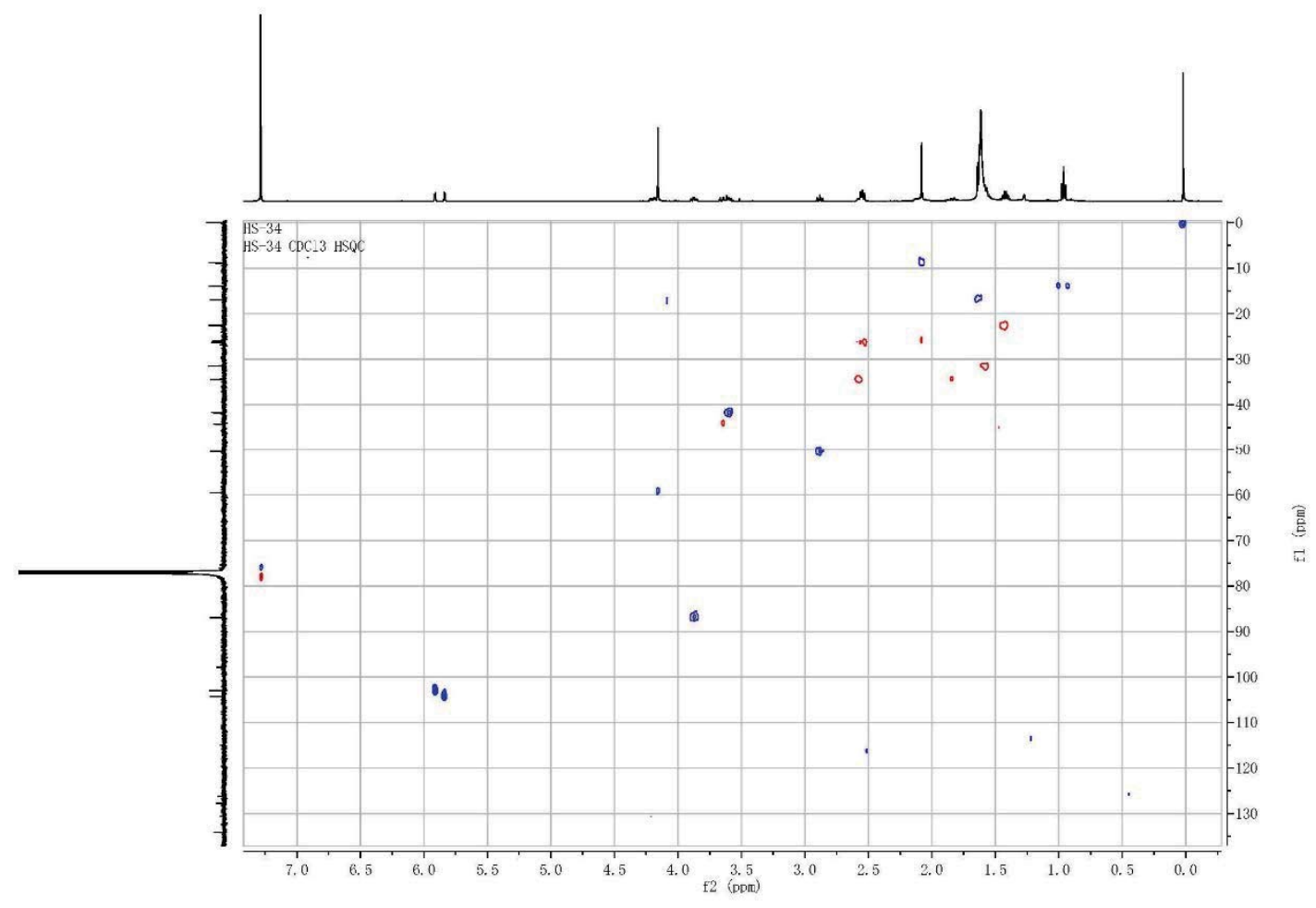

S27: HMBC of 10-epi-3-n-butylneostemonine (4)

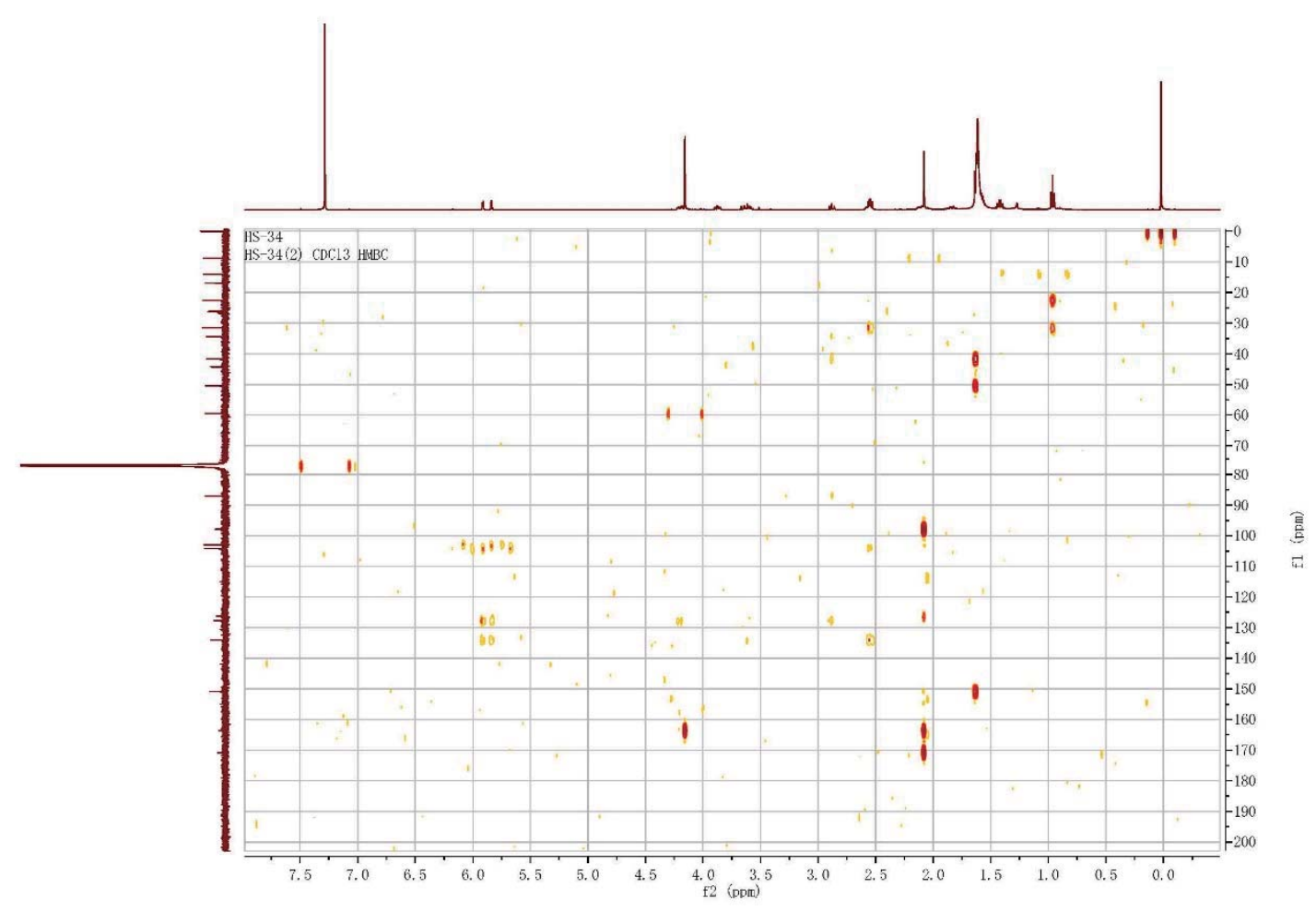


S28: ${ }^{1} \mathrm{H}^{1} \mathrm{H}$ COSY of 10-epi-3-n-butylneostemonine (4)

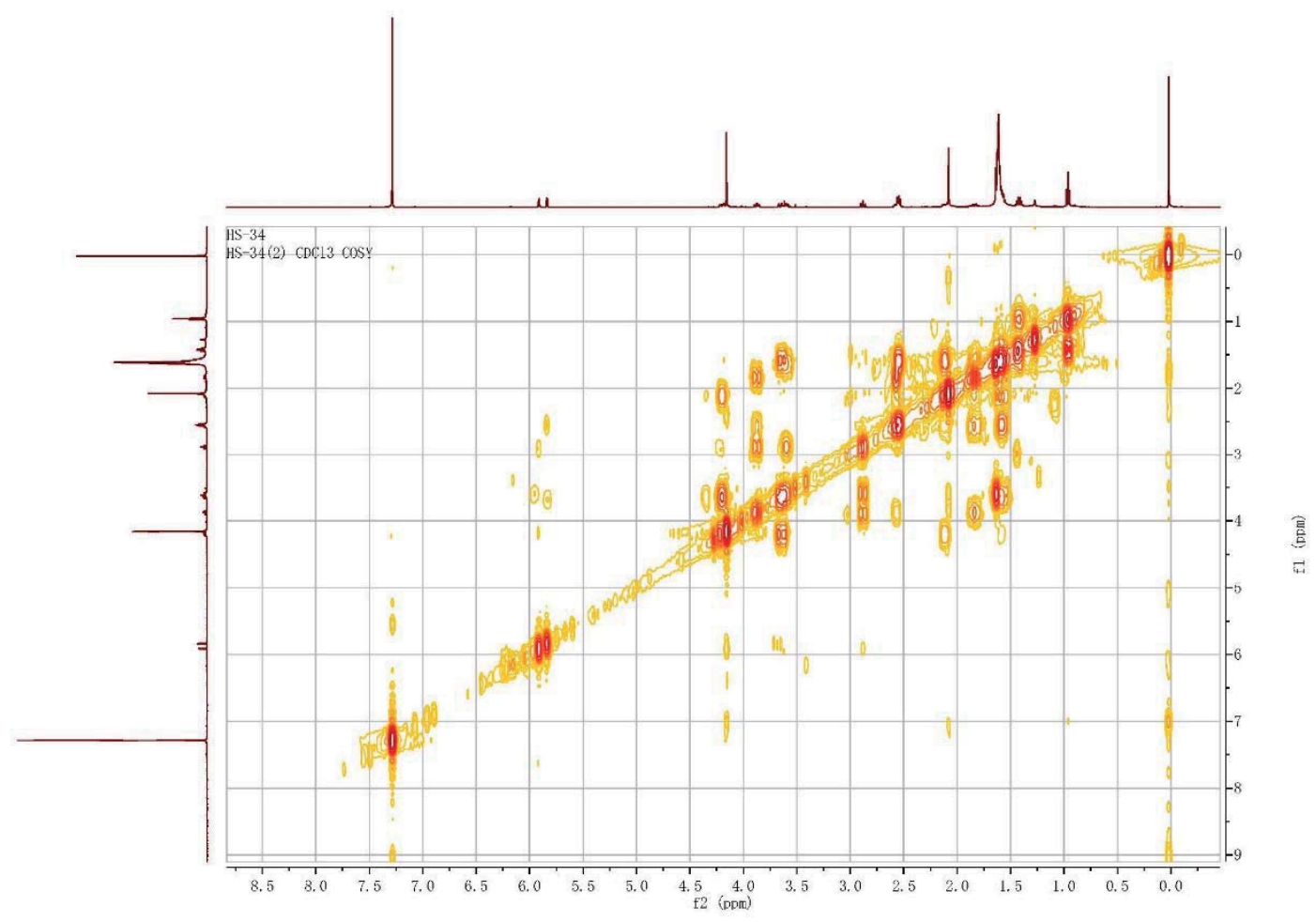

S29: ROESY of 10-epi-3-n-butylneostemonine (4)

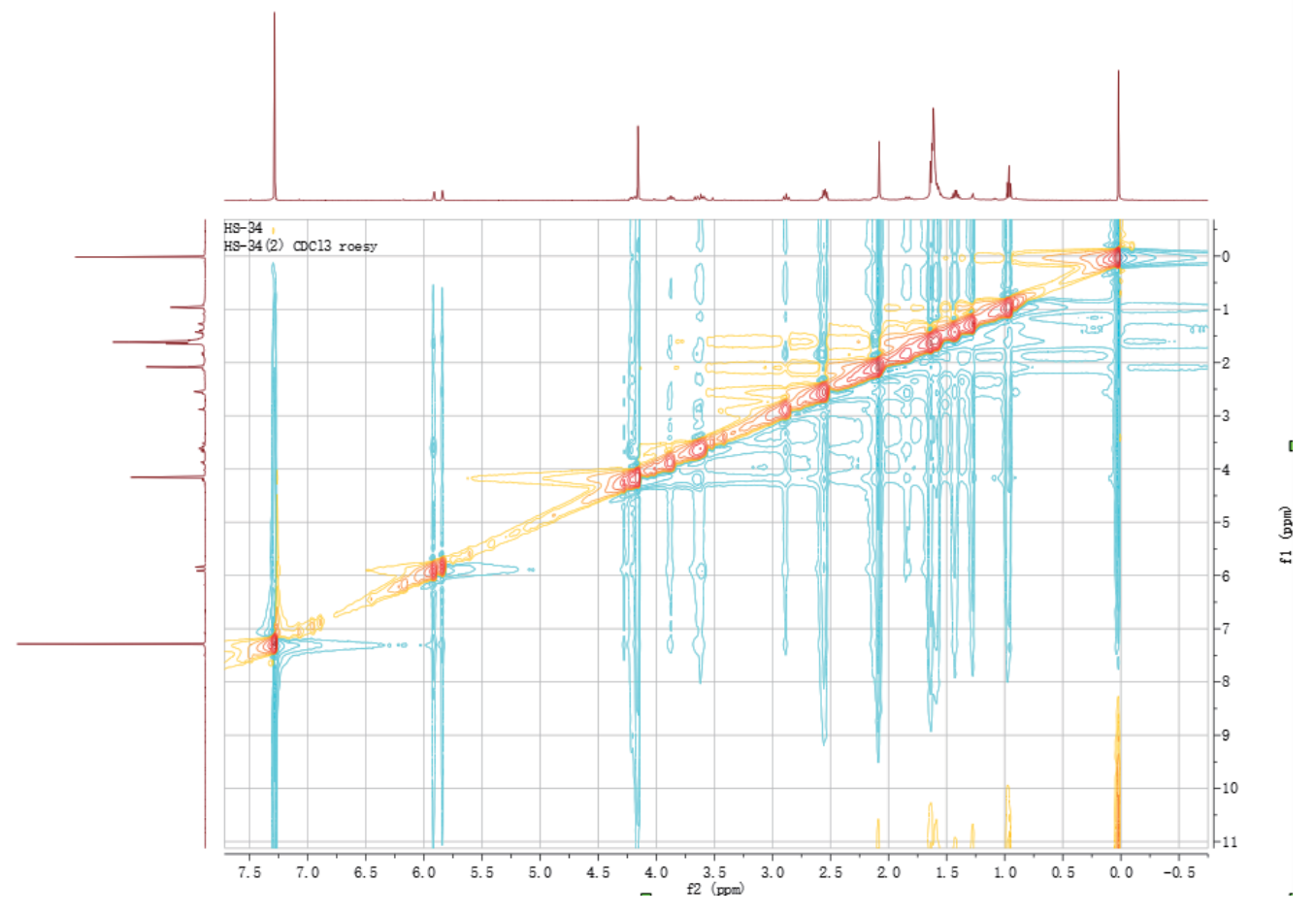


S30: EIMS of 10-epi-3-n-butylneostemonine (4)

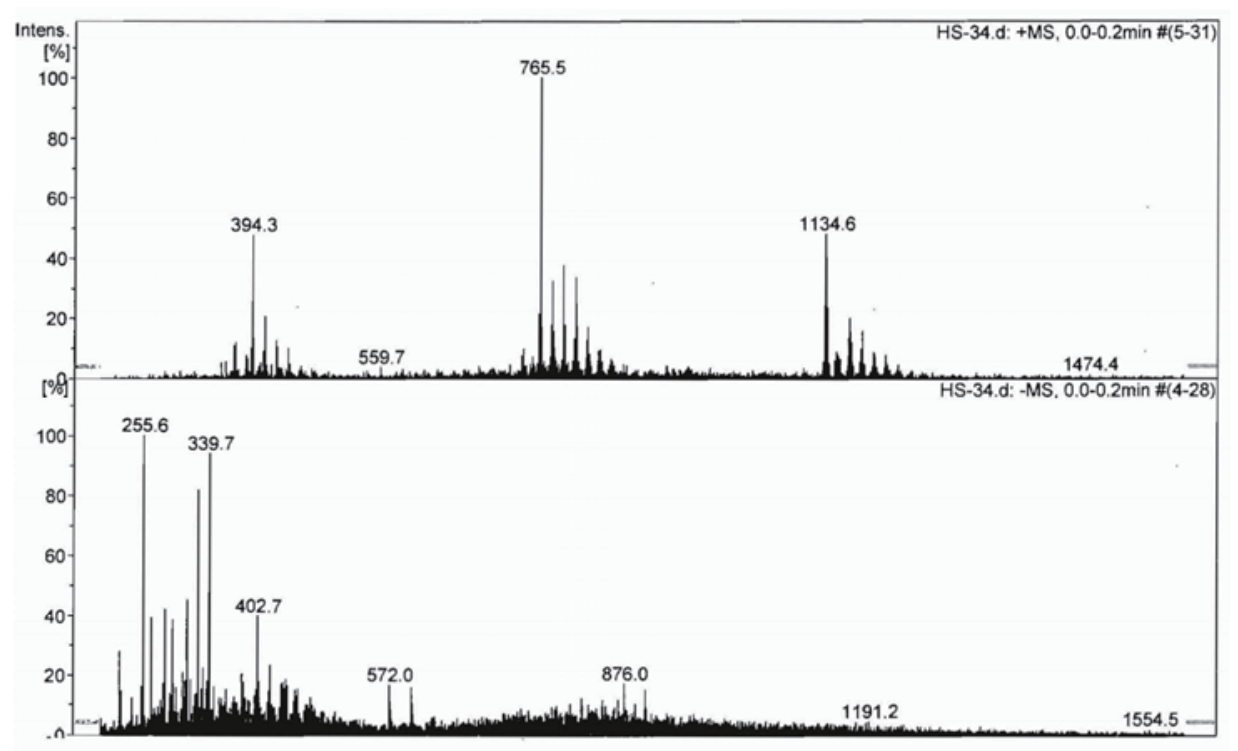


S31: ${ }^{1} \mathrm{H}$ NMR of 8-oxo-oxymaistemonine (5)

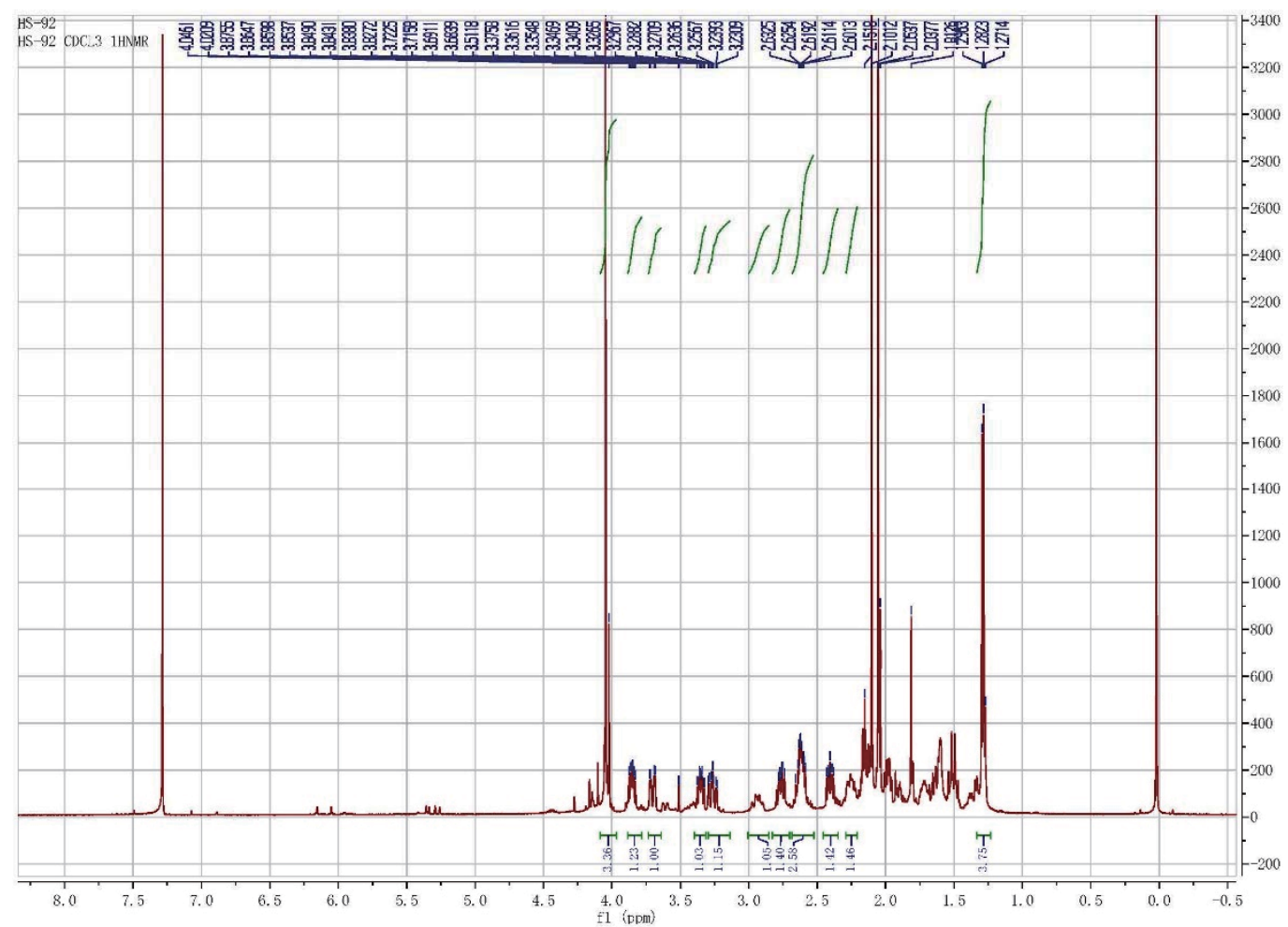

S32: DEPT of 8-oxo-oxymaistemonine (5)

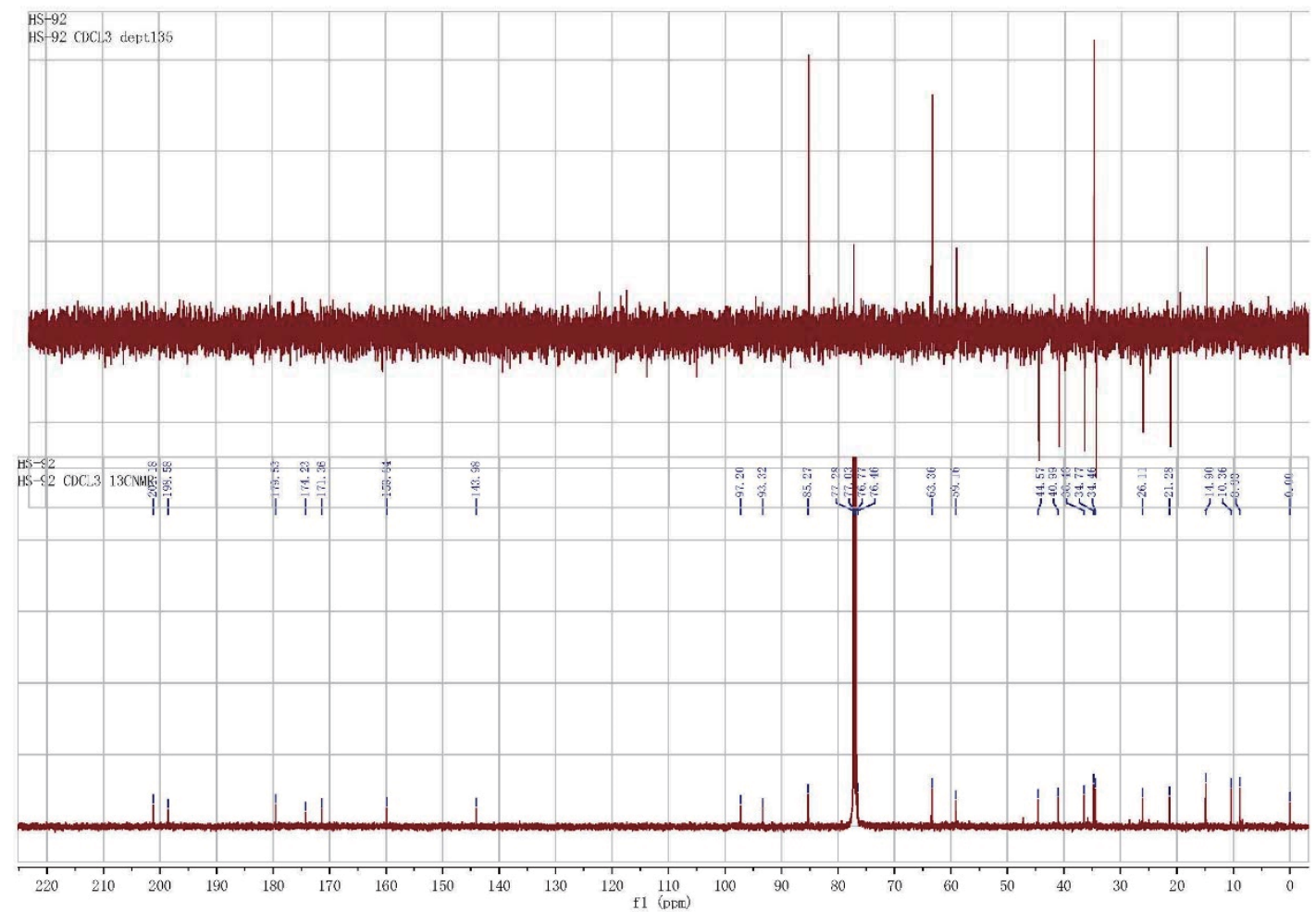


S33: HSQC of 8-oxo-oxymaistemonine (5)

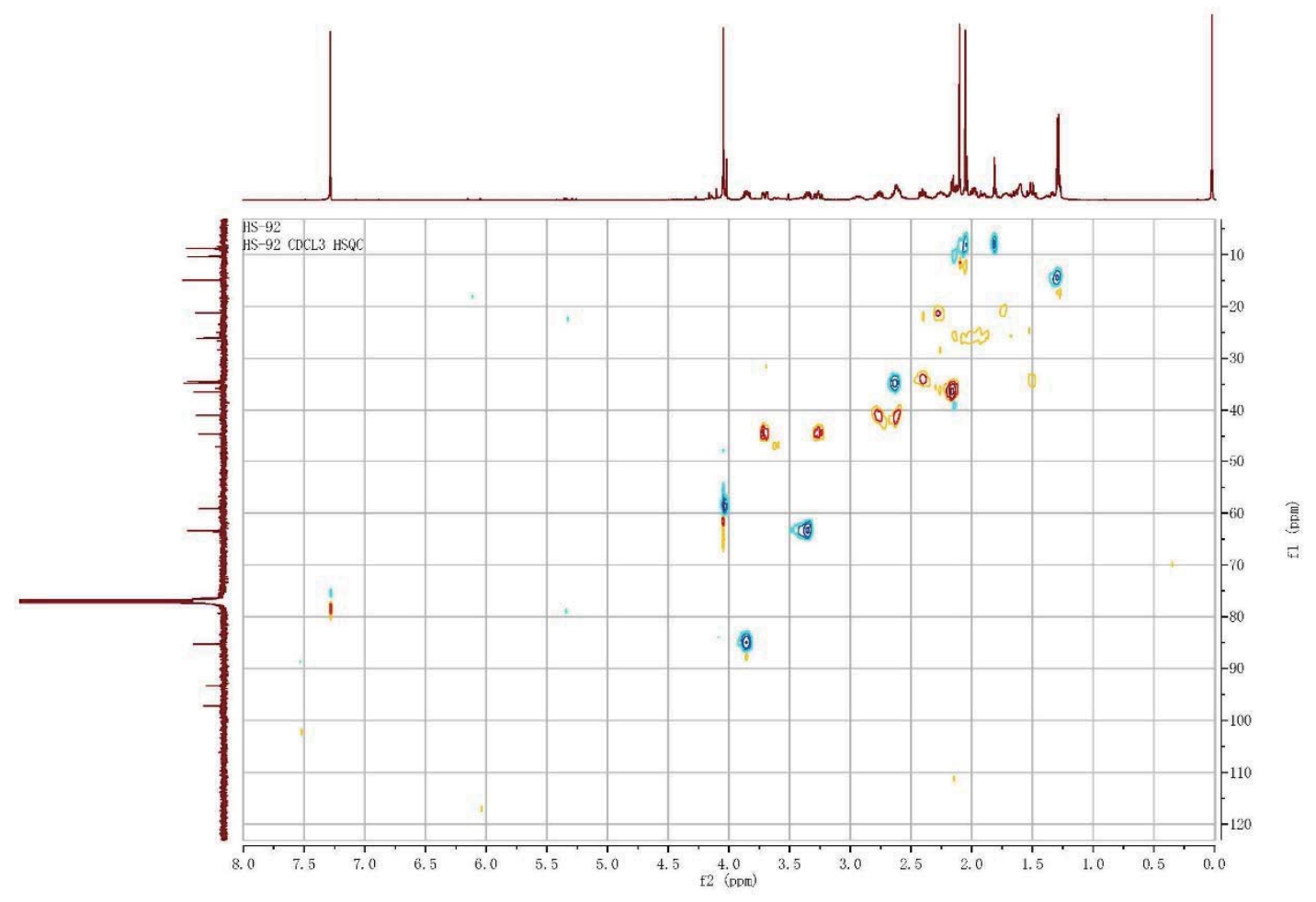

S34: HMBC of 8-oxo-oxymaistemonine (5)

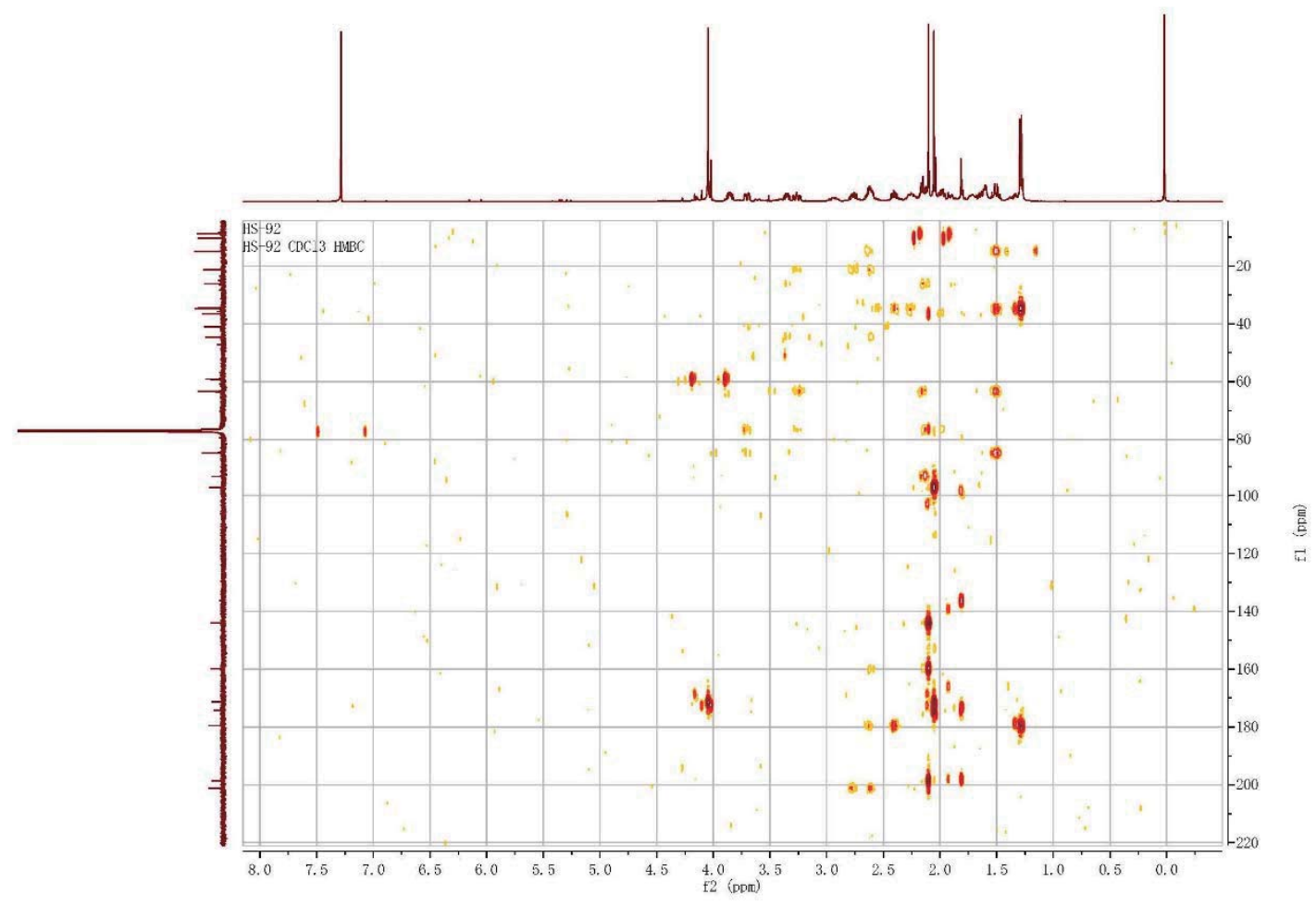


S35: ${ }^{1} \mathrm{H}^{1} \mathrm{H}$ COSY of 8-oxo-oxymaistemonine (5)

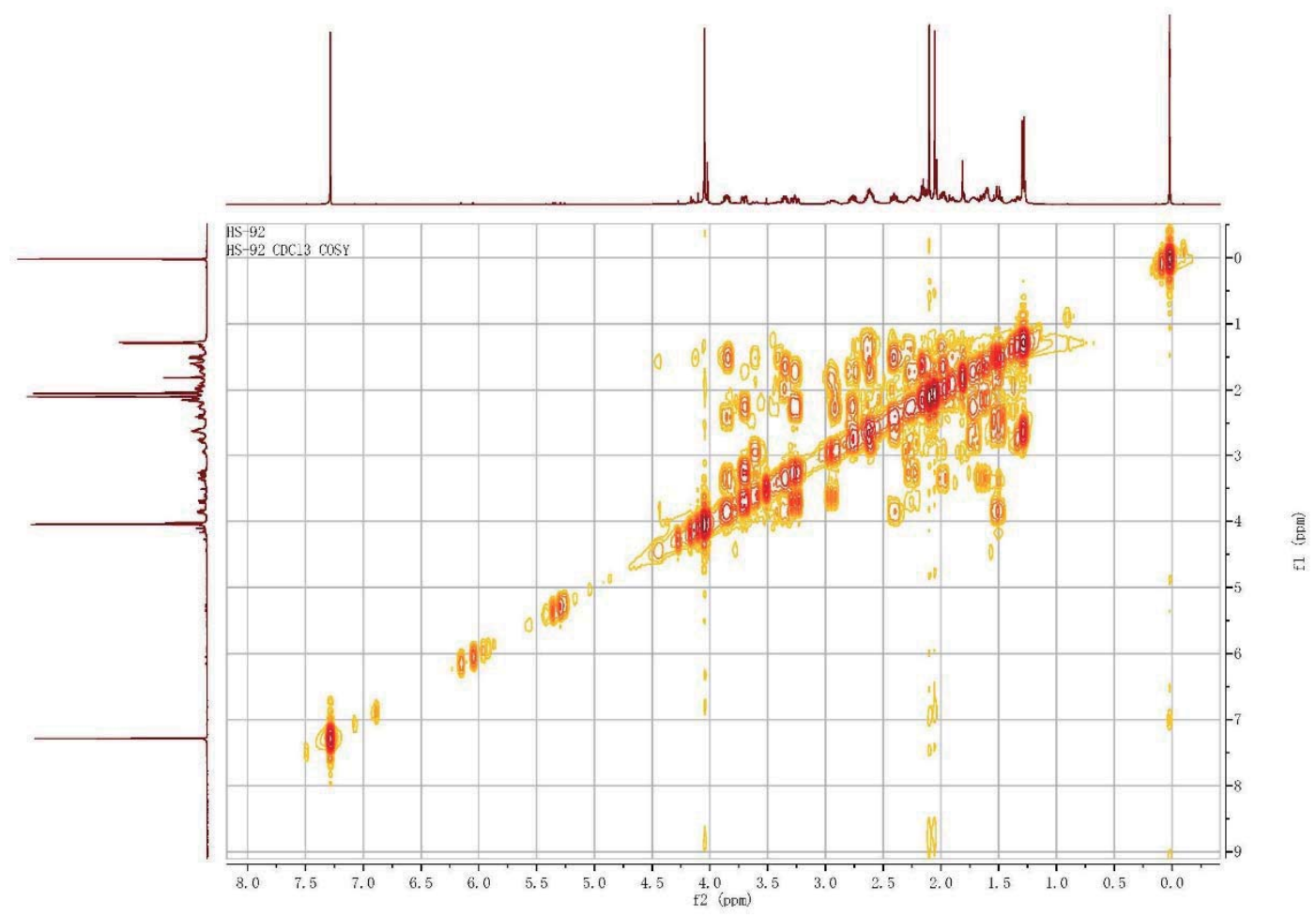

S36: ROESY of 8-oxo-oxymaistemonine (5)

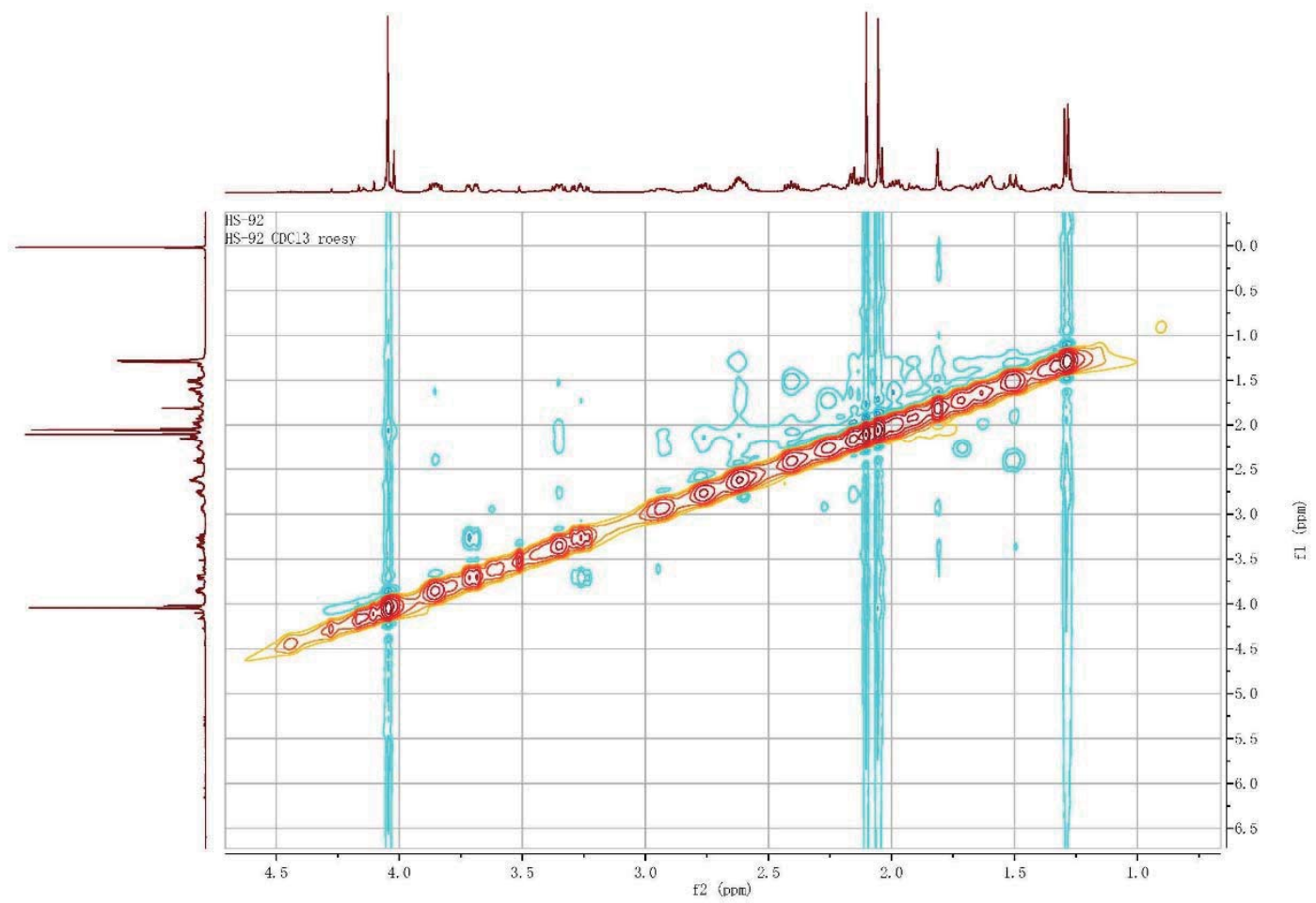


S37: ESIMS of 8-oxo-oxymaistemonine (5)

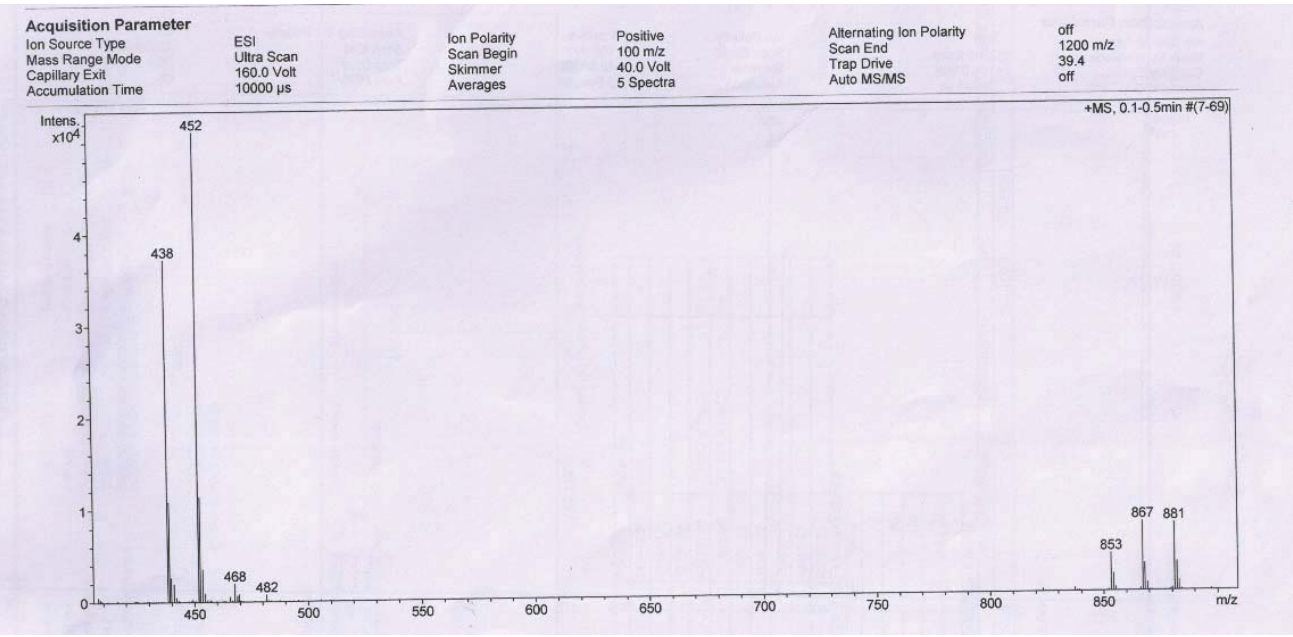


S38: ${ }^{1} \mathrm{H}$ NMR of protostemonine N4-oxide (6)

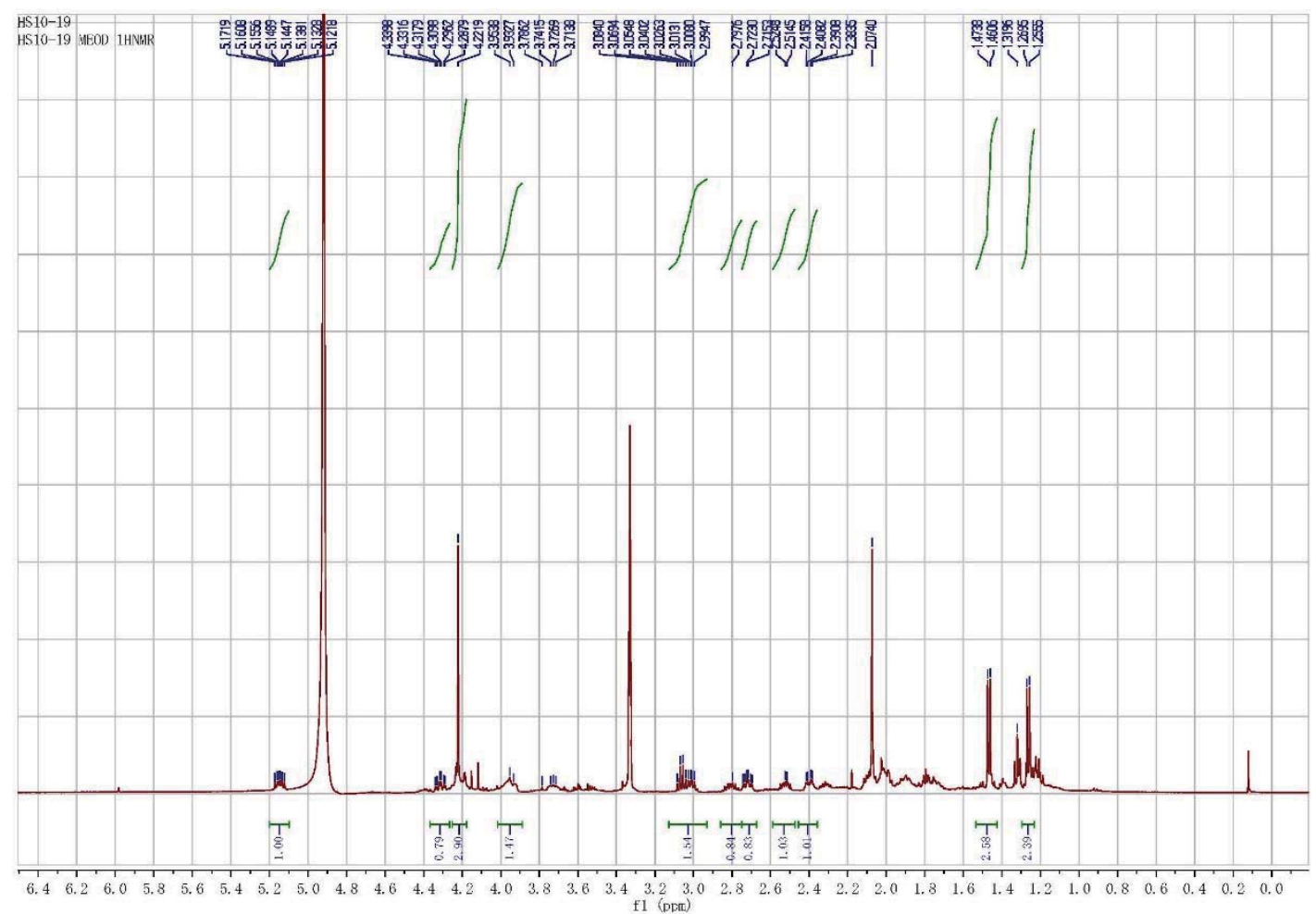

S39: DEPT of protostemonine $\mathrm{N}_{4}$-oxide (6)

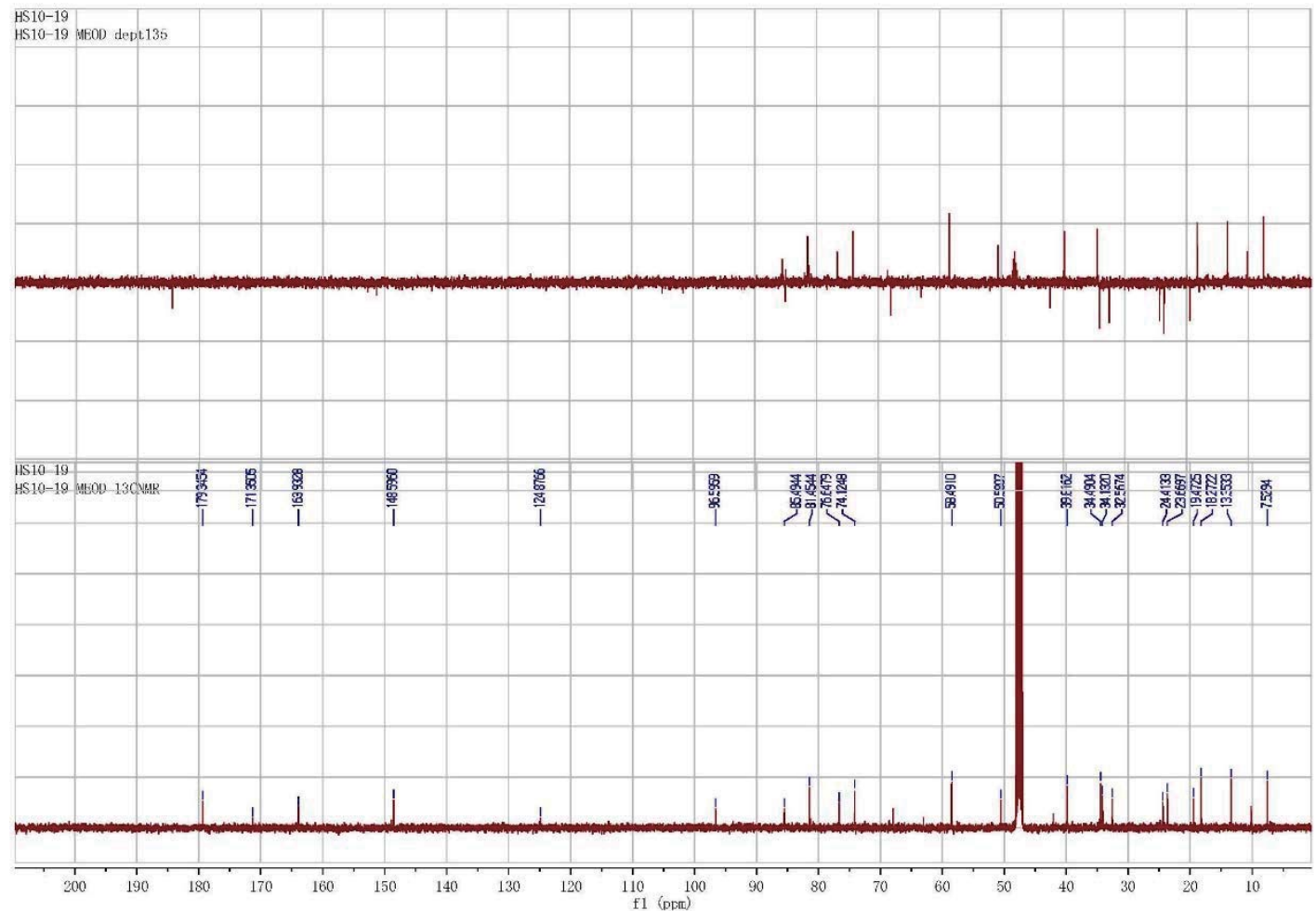


S40: HSQC of protostemonine N4-oxide (6)

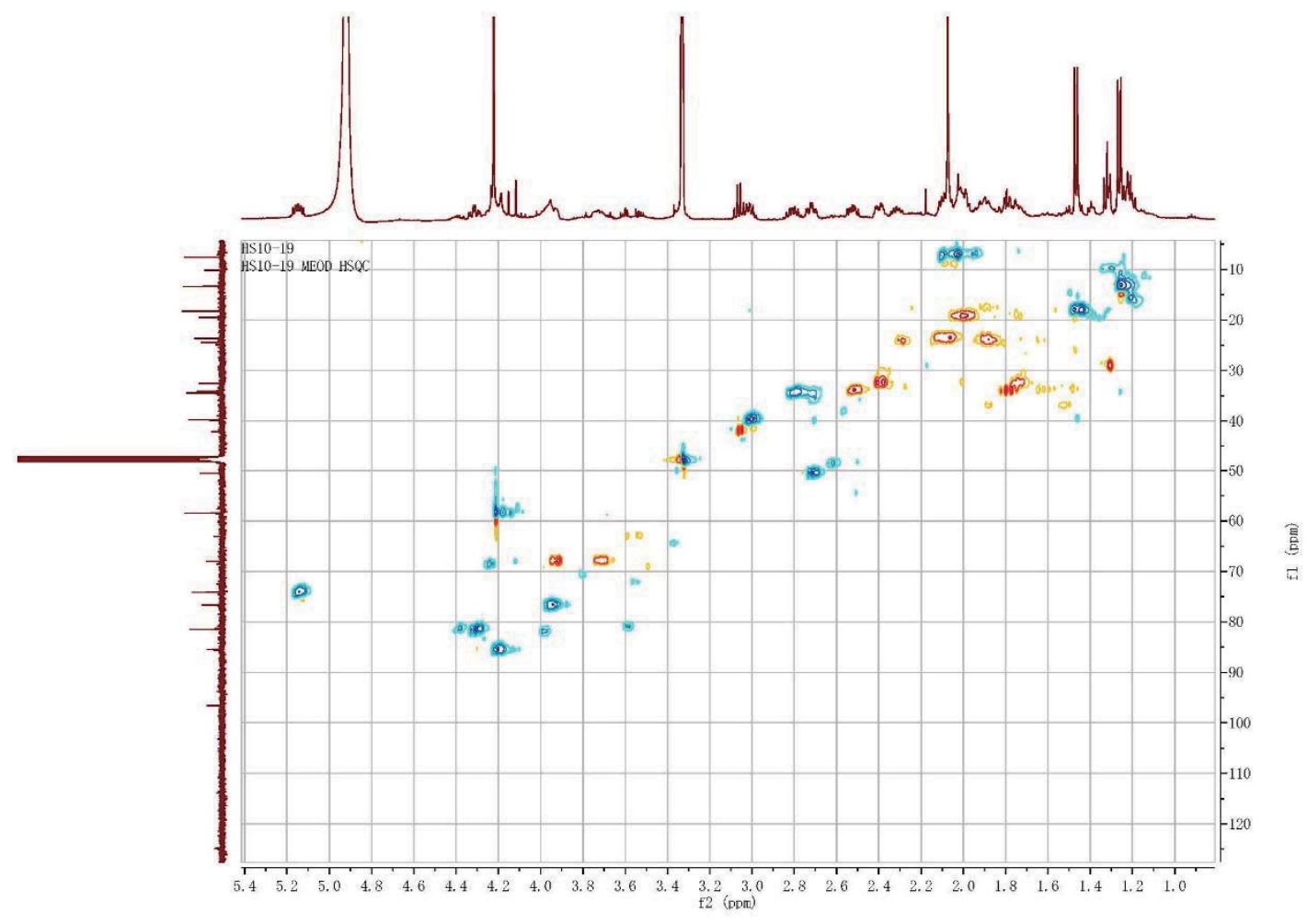

S41: HMBC of protostemonine $\mathrm{N}_{4}$-oxide (6)

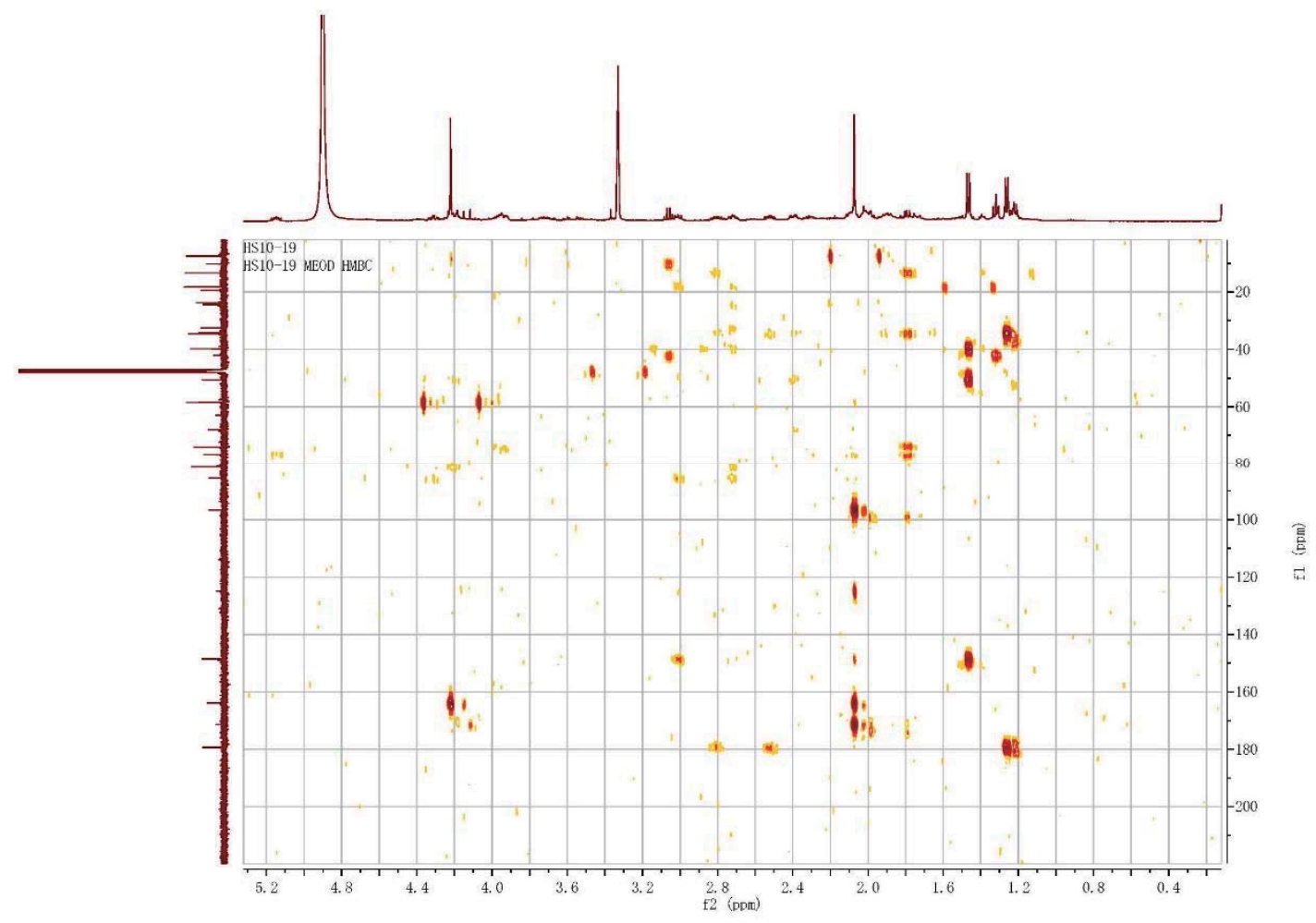


S42: ${ }^{1} \mathrm{H}^{1} \mathrm{H}$ COSY of protostemonine $\mathrm{N}_{4}$-oxide (6)

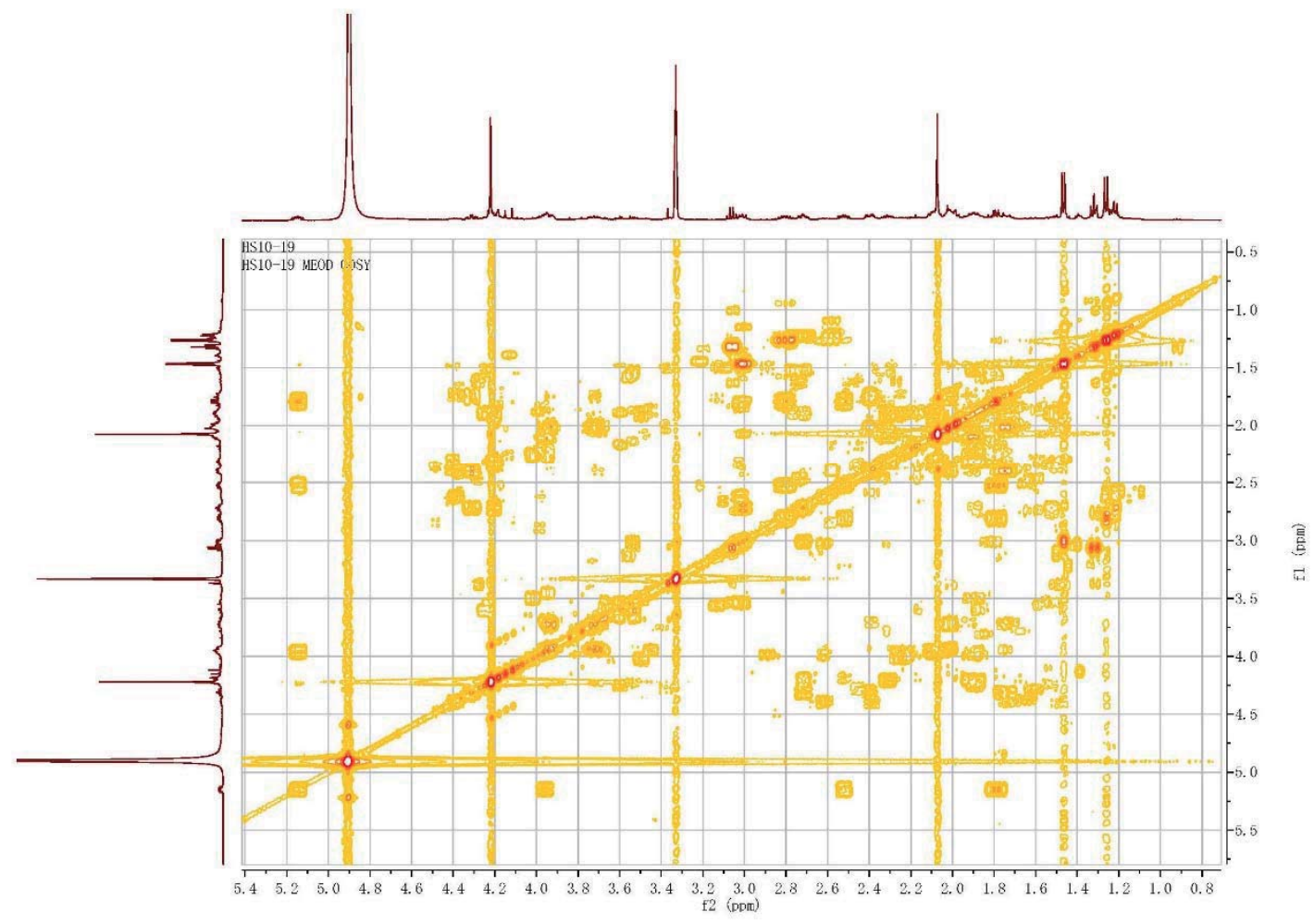

S43: ROESY of protostemonine $\mathrm{N}_{4}$-oxide(6)

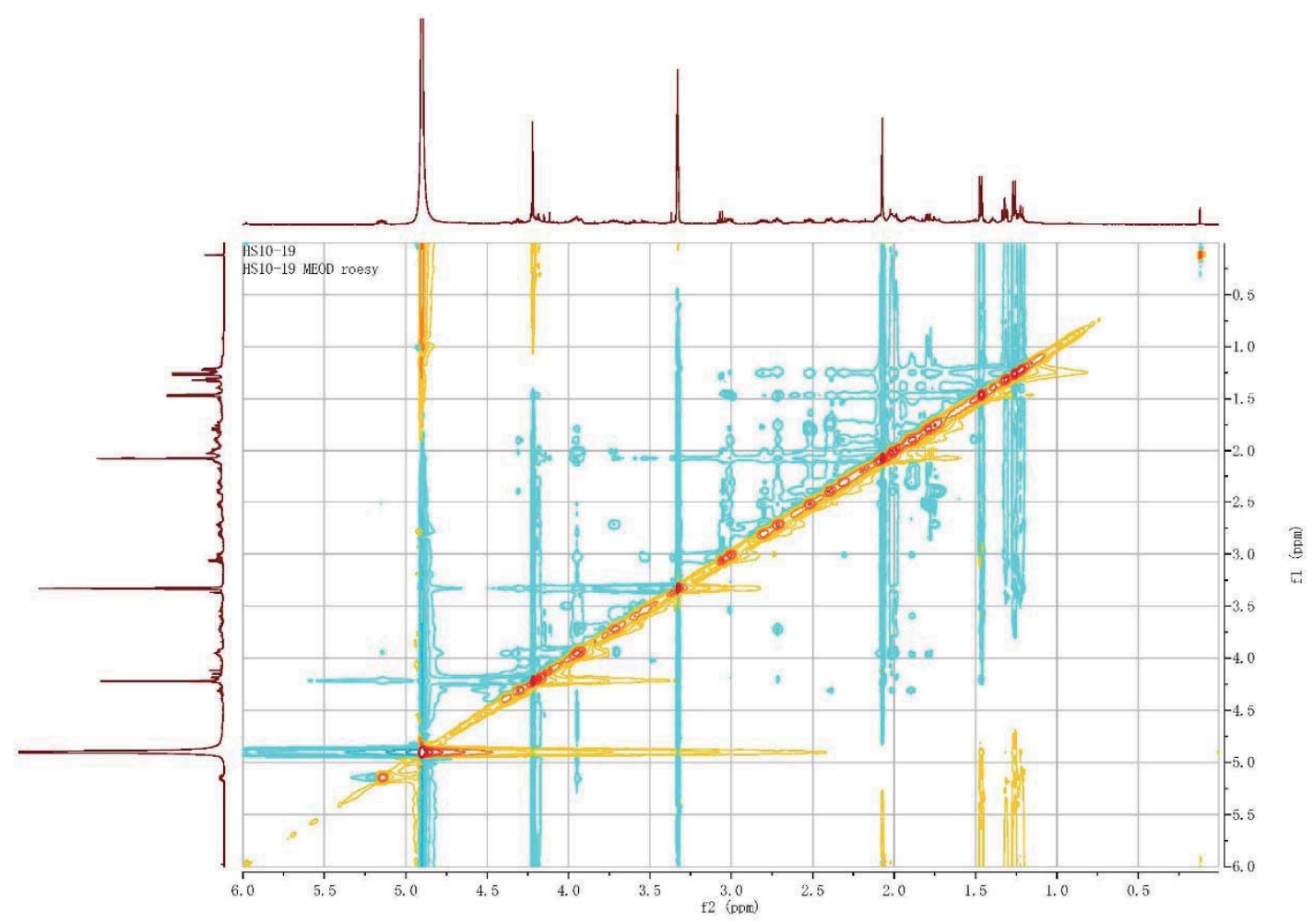


S44: ESIMS of protostemonine N4-oxide (6)

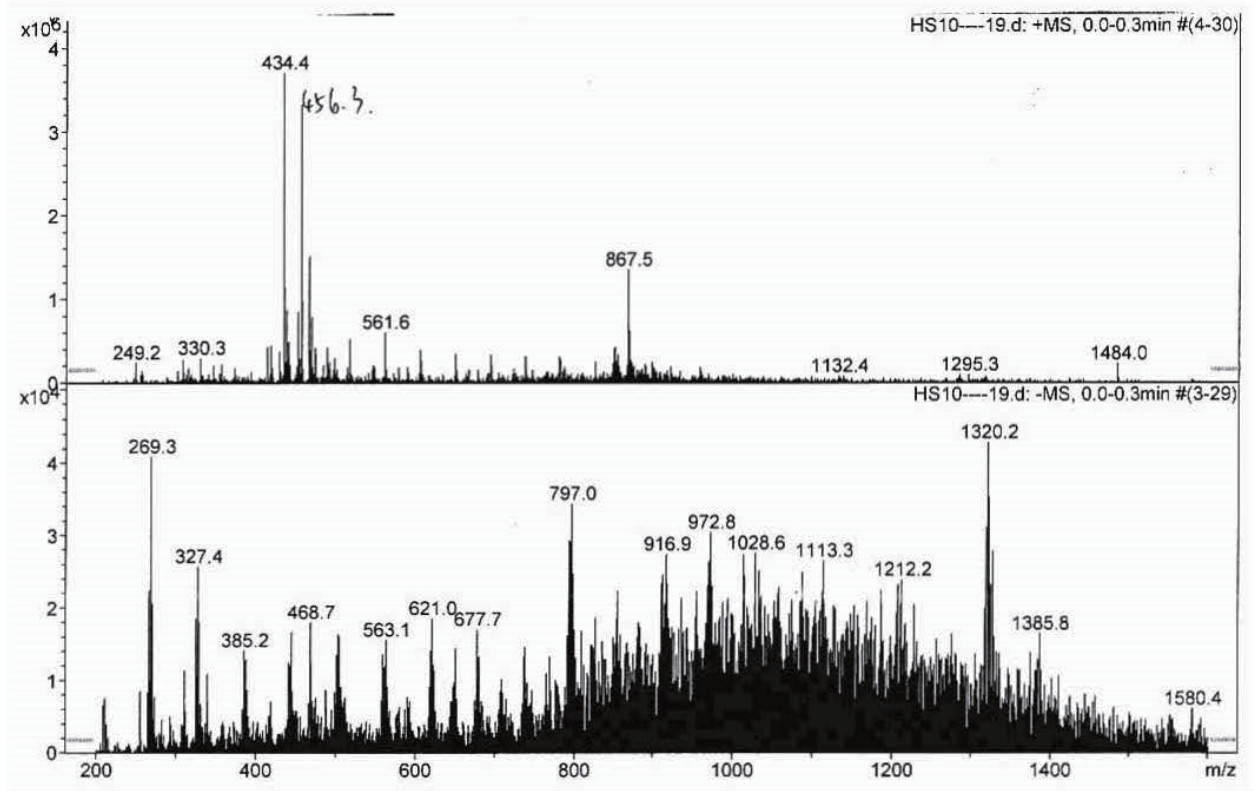


S45: ${ }^{1} \mathrm{H}$ NMR of (19S)-hydroxy-21-methoxystemofoline (7)

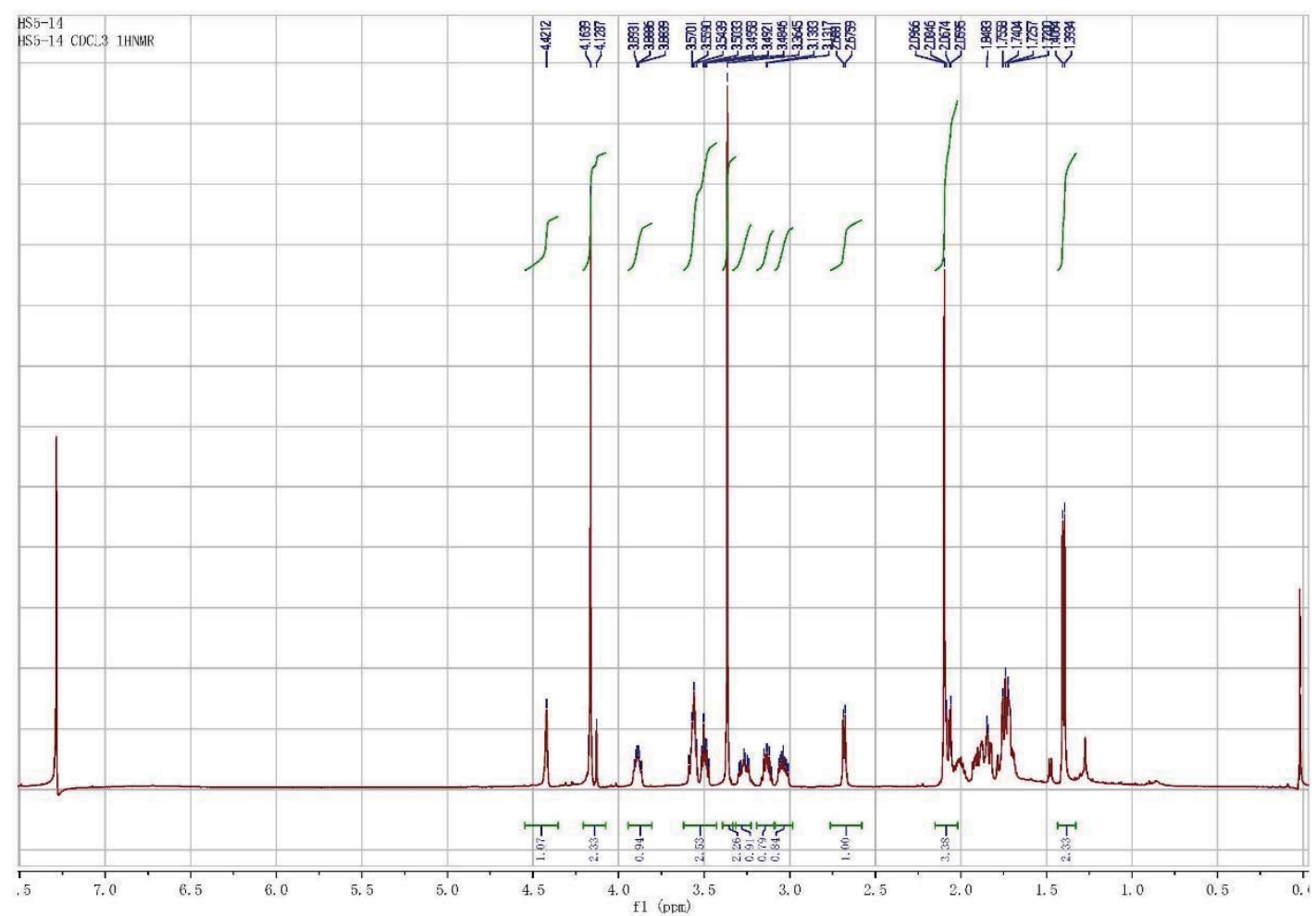

S46: DEPT of (19S)-hydroxy-21-methoxystemofoline (7)

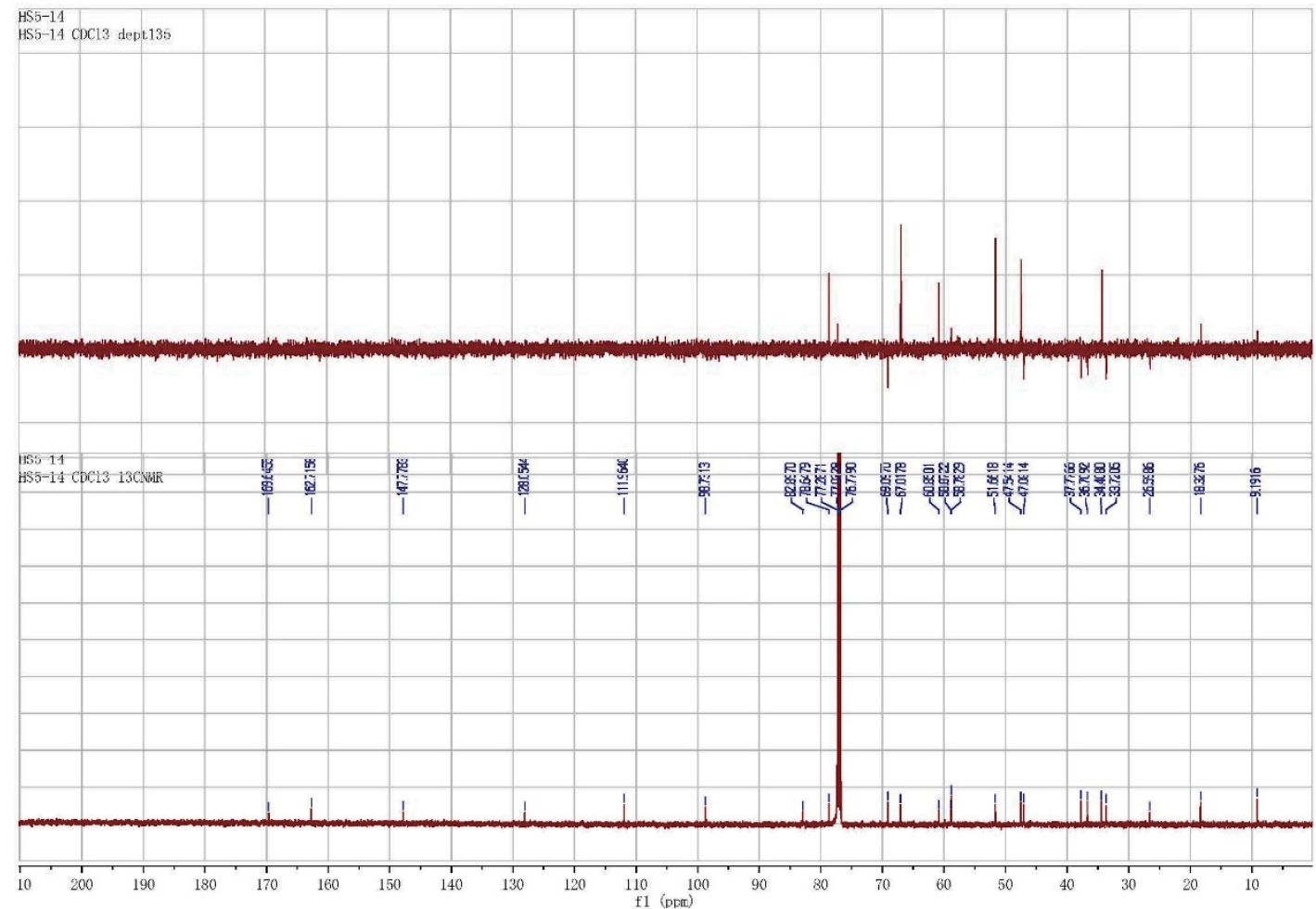


S47: HSQC of (19S)-hydroxy-21-methoxystemofoline (7)

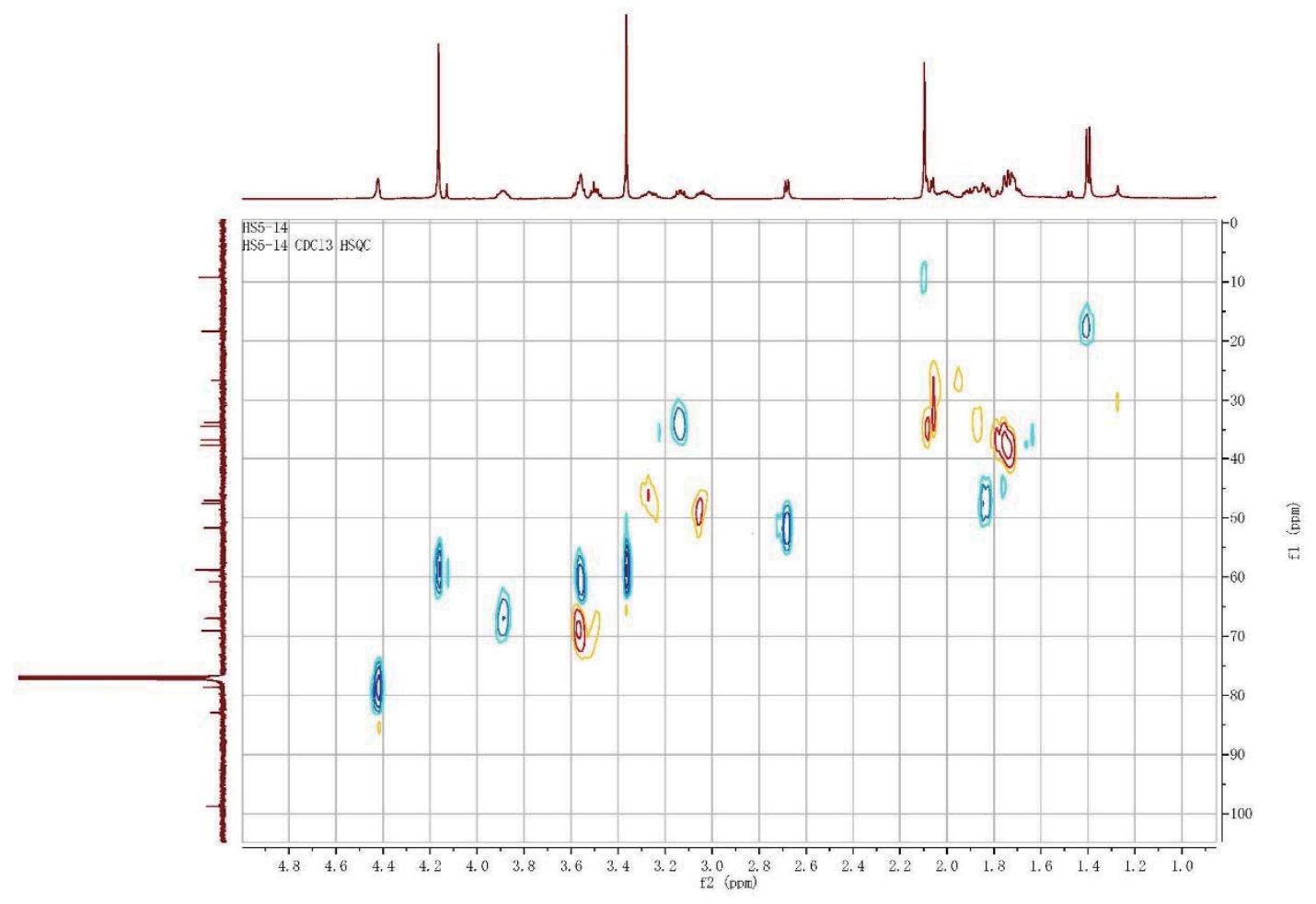

S48: HMBC of (19S)-hydroxy-21-methoxystemofoline (7)

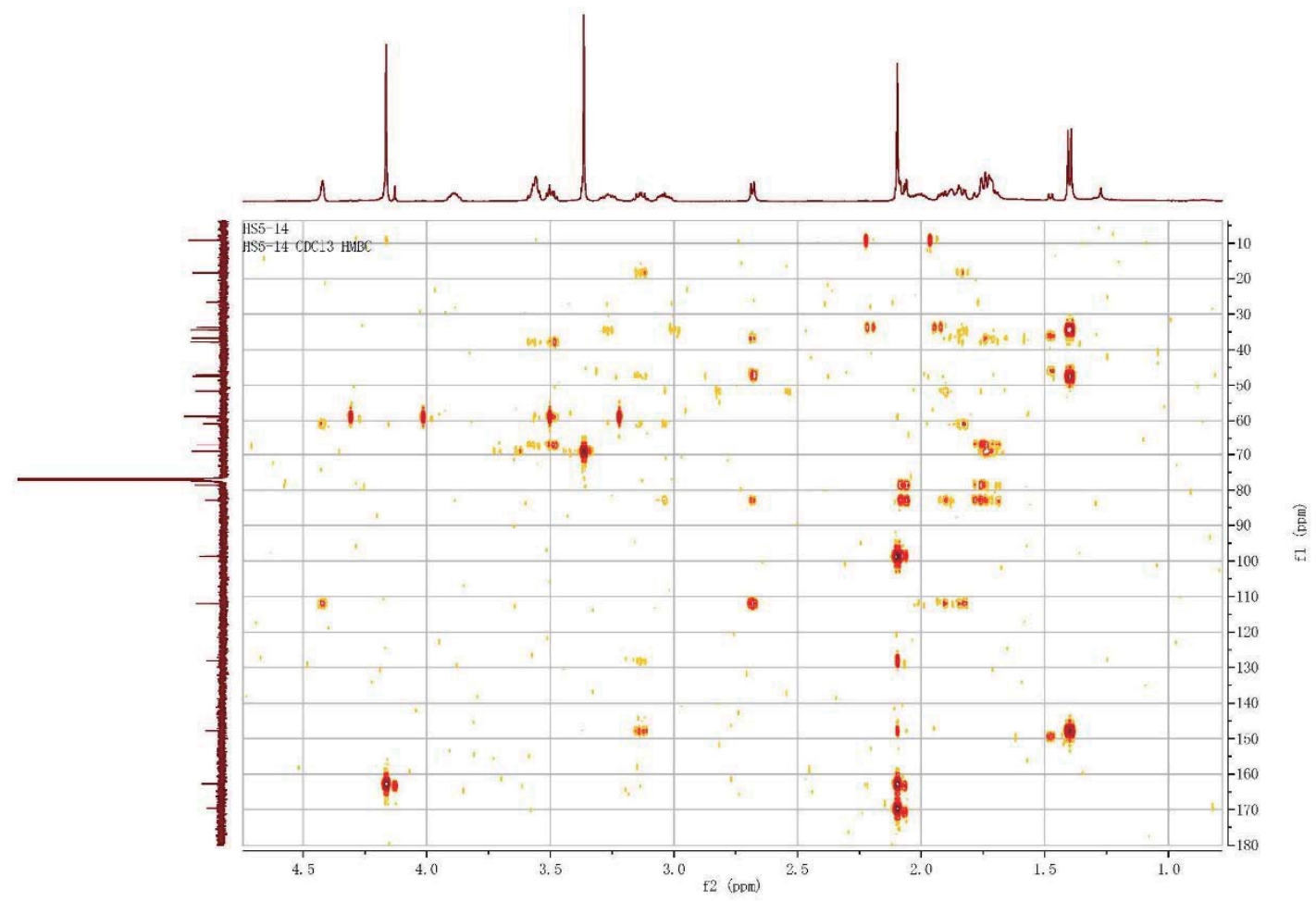


S49: ${ }^{1} \mathrm{H}{ }^{1} \mathrm{H}$ COSY of (19S)-hydroxy-21-methoxystemofoline (7)

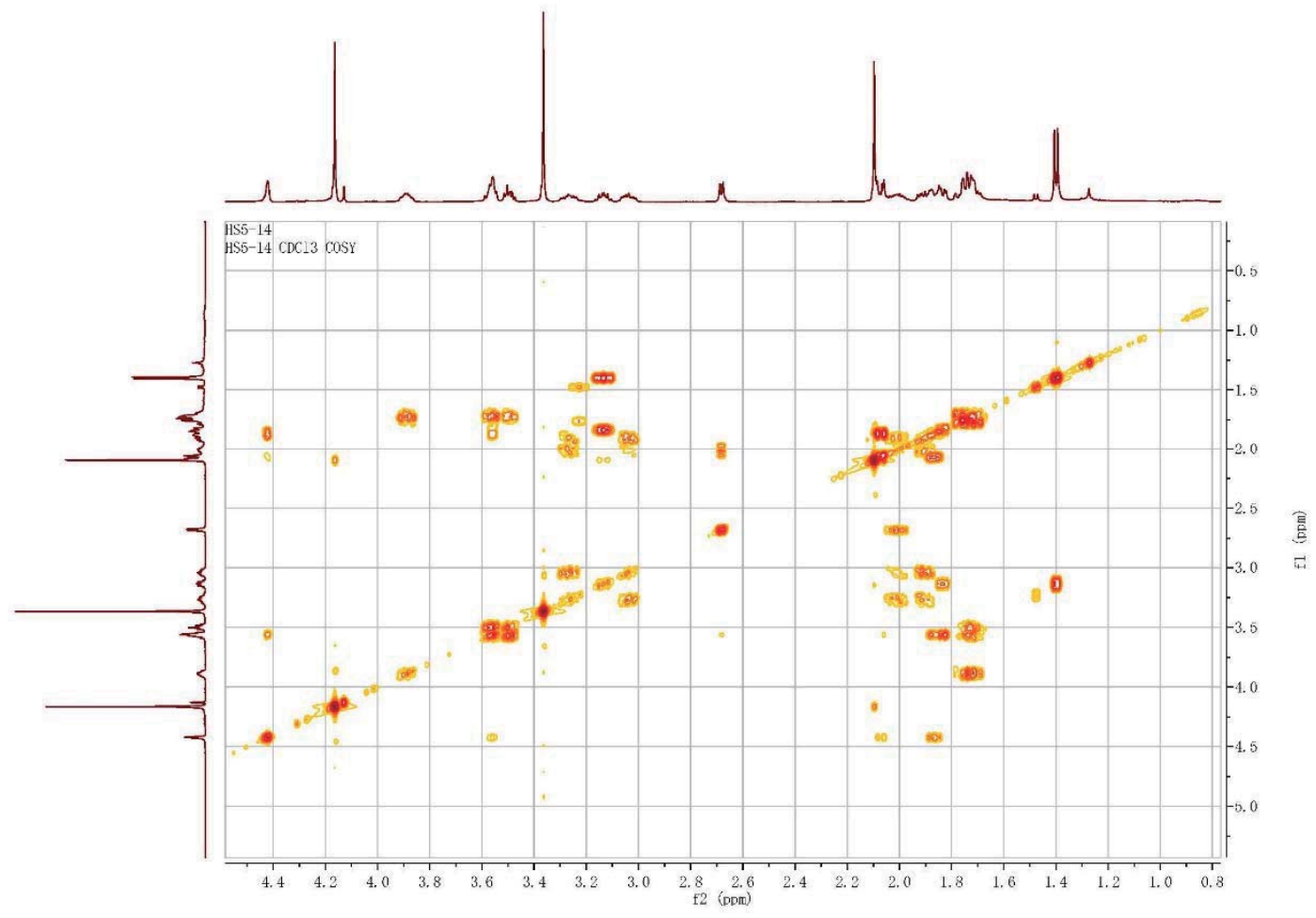

S50: ROESY of (19S)-hydroxy-21-methoxystemofoline (7)

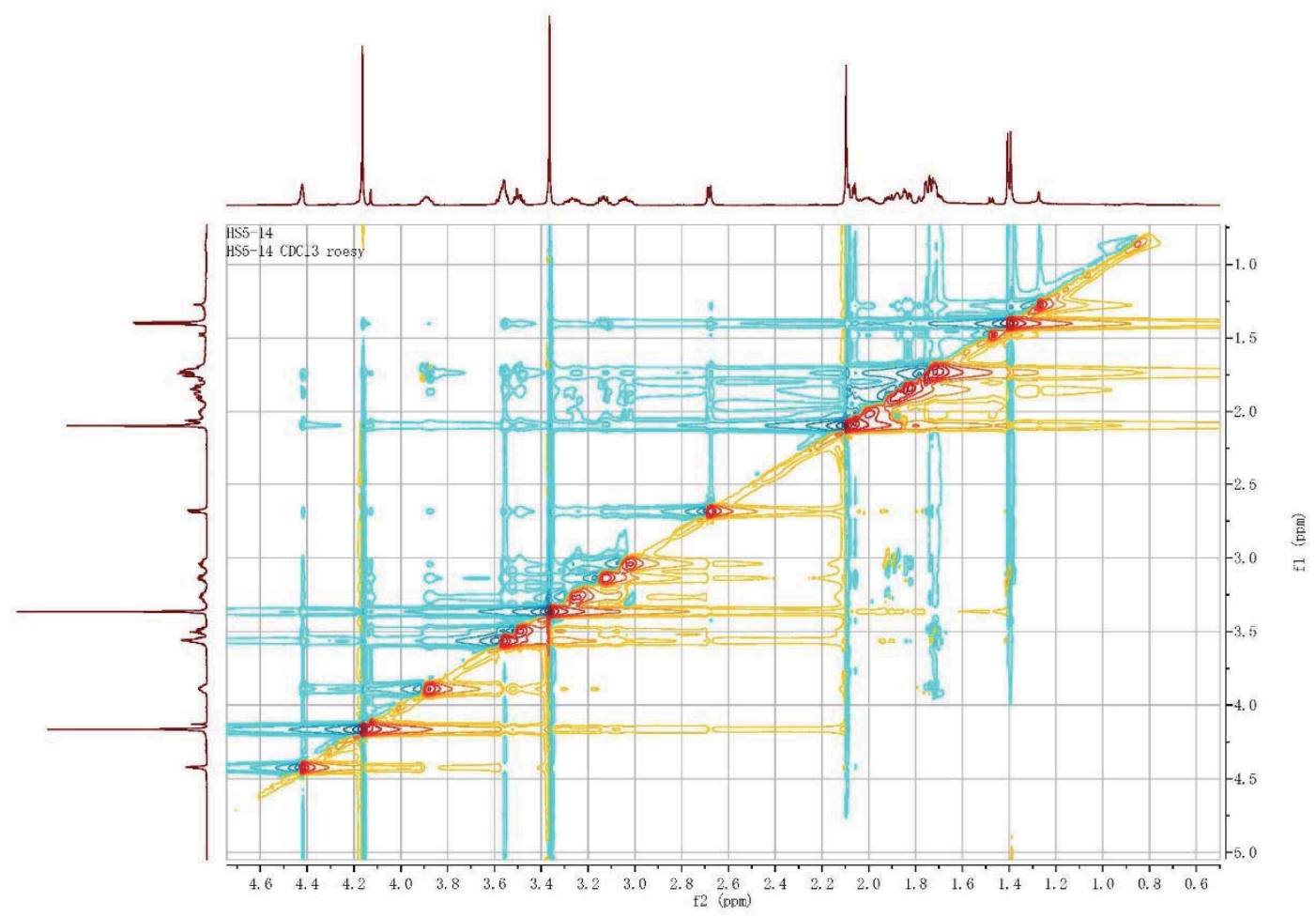


S51: ESIMS of (19S)-hydroxy-21-methoxystemofoline (7)

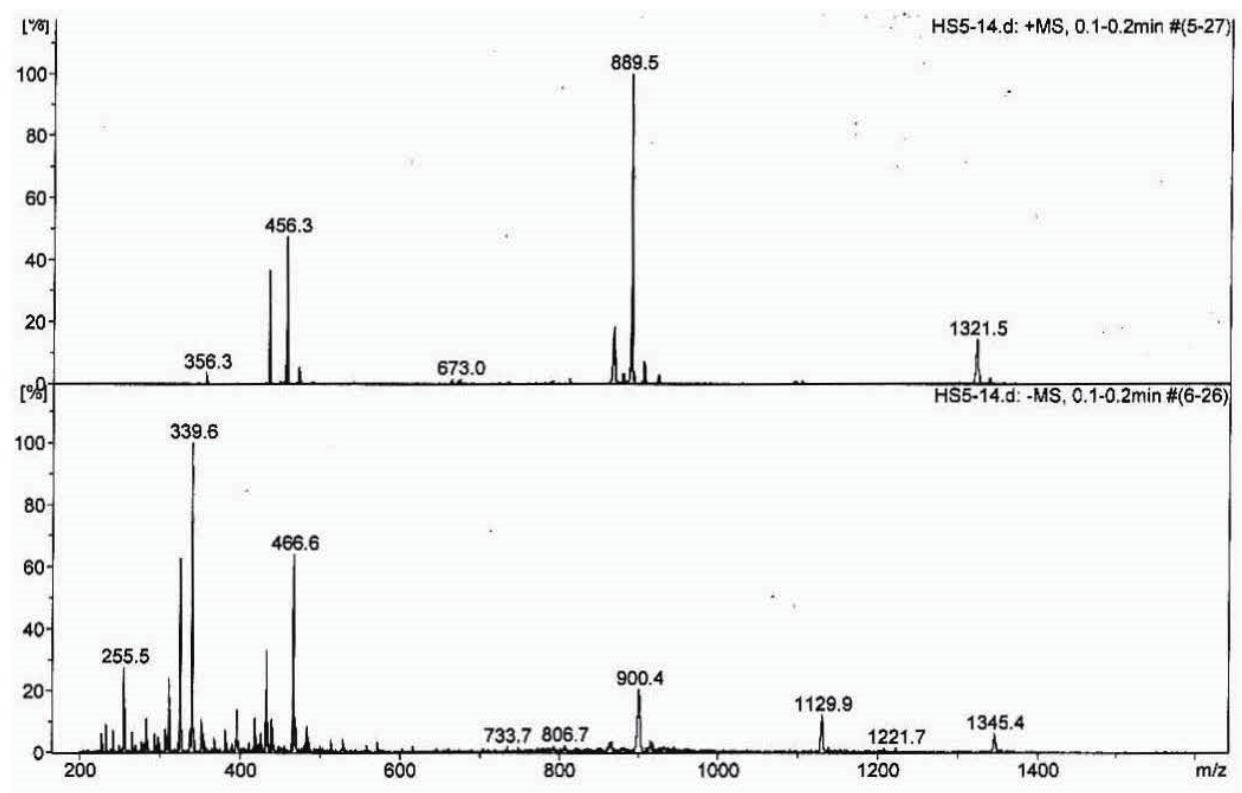


S52: ${ }^{1} \mathrm{H}$ NMR of parvistemonine A (8)

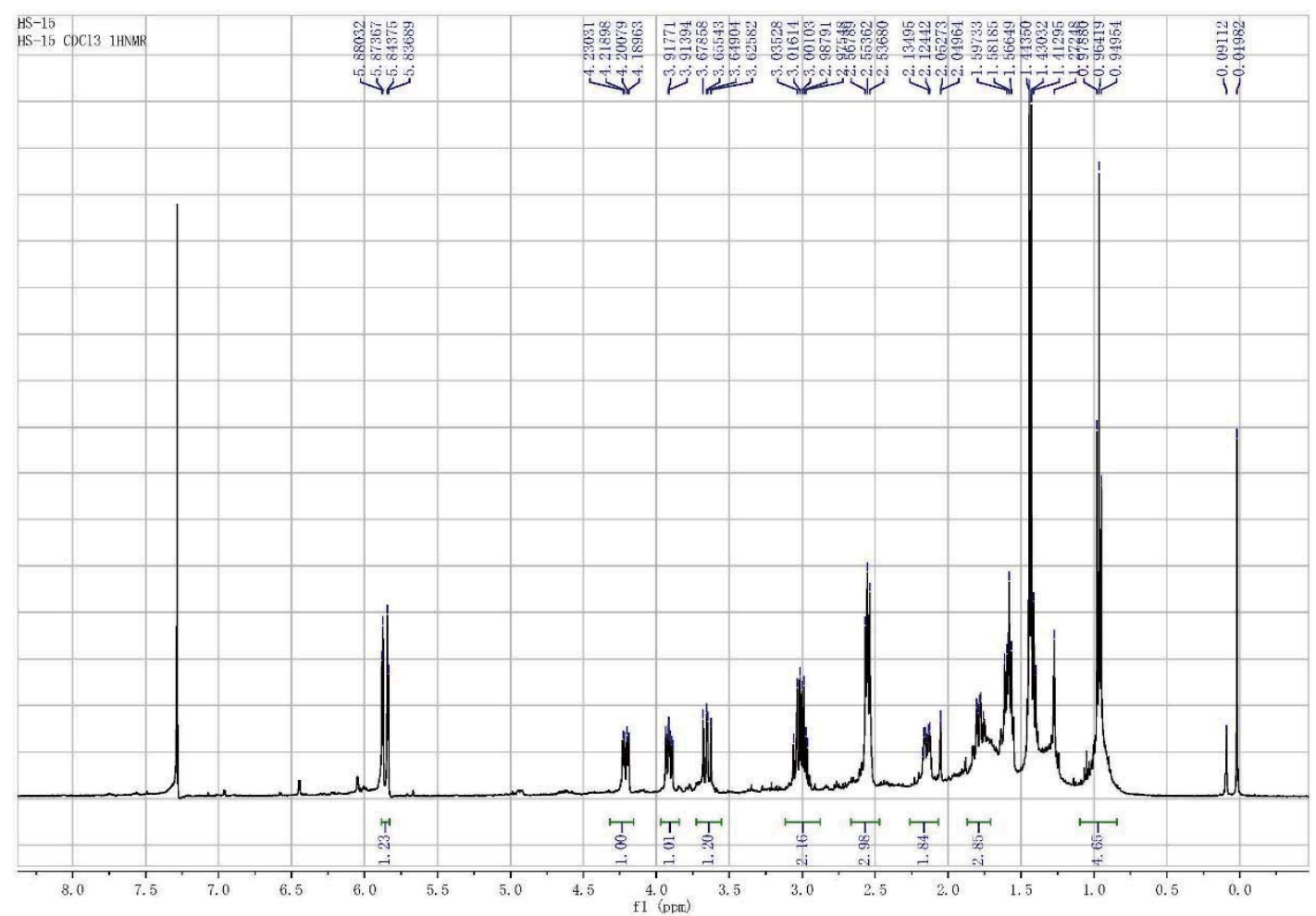

S53: DEPT of parvistemonine A (8)

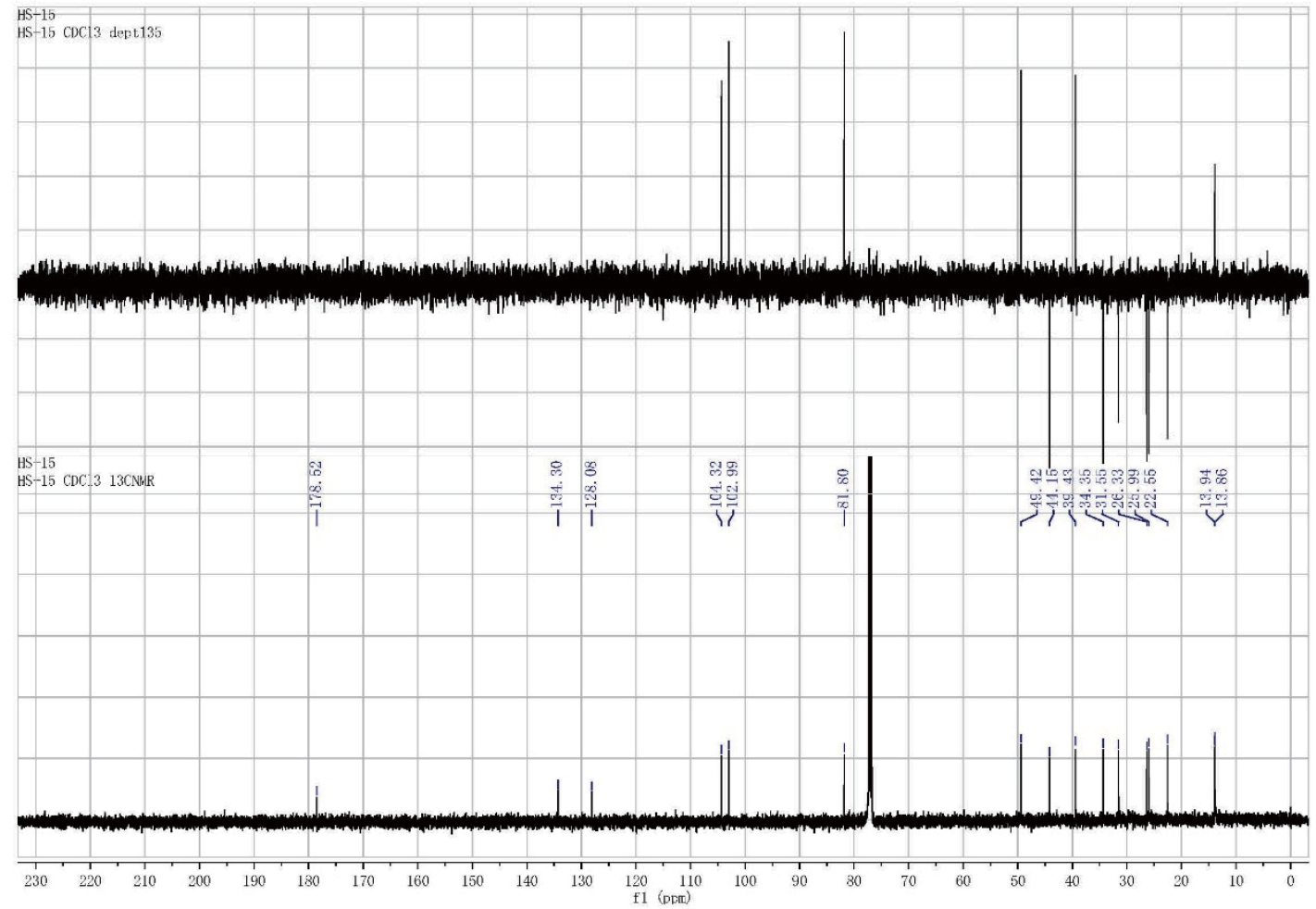


S54: HSQC of parvistemonine A (8)

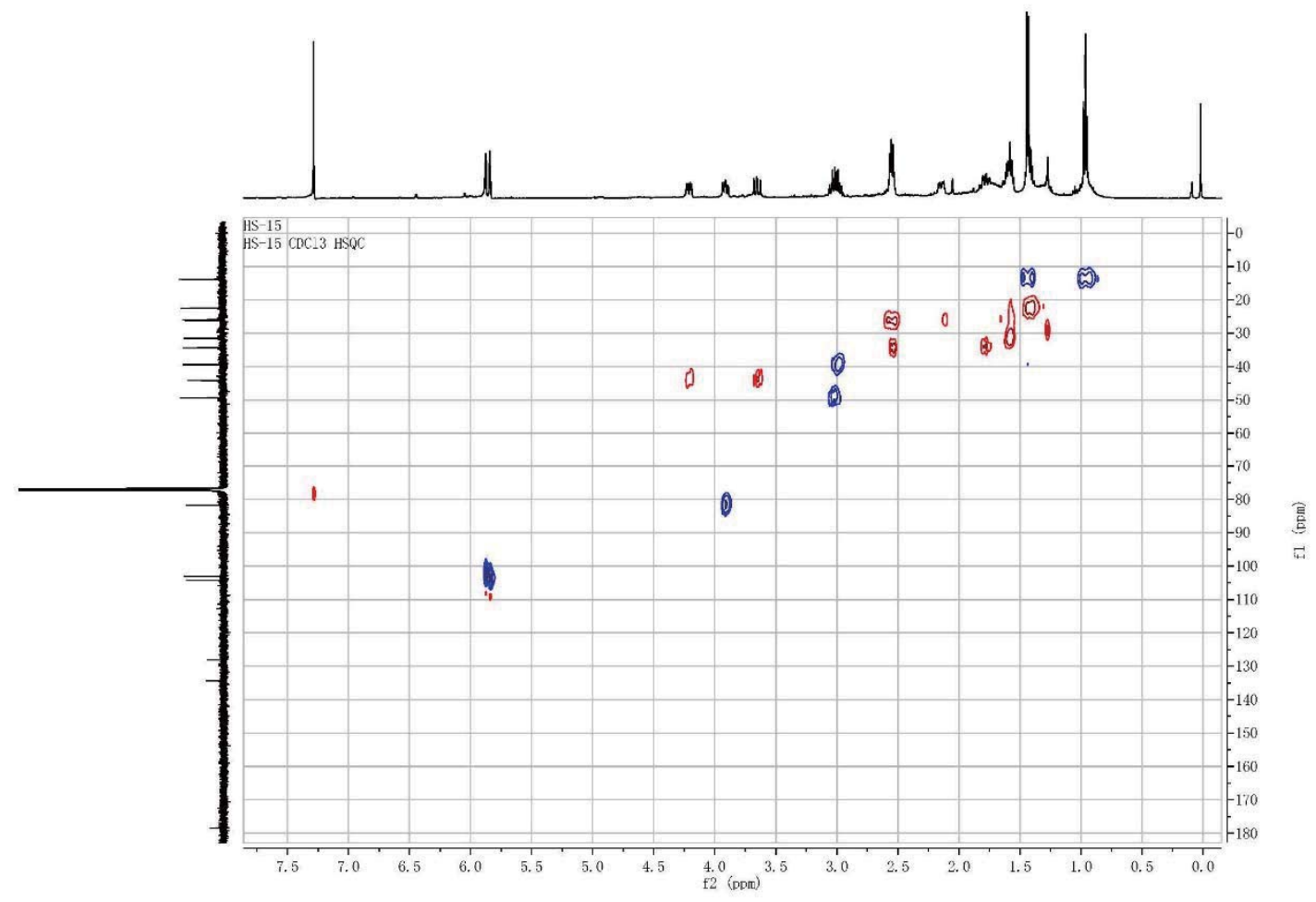

S55: HMBC of parvistemonine A (8)

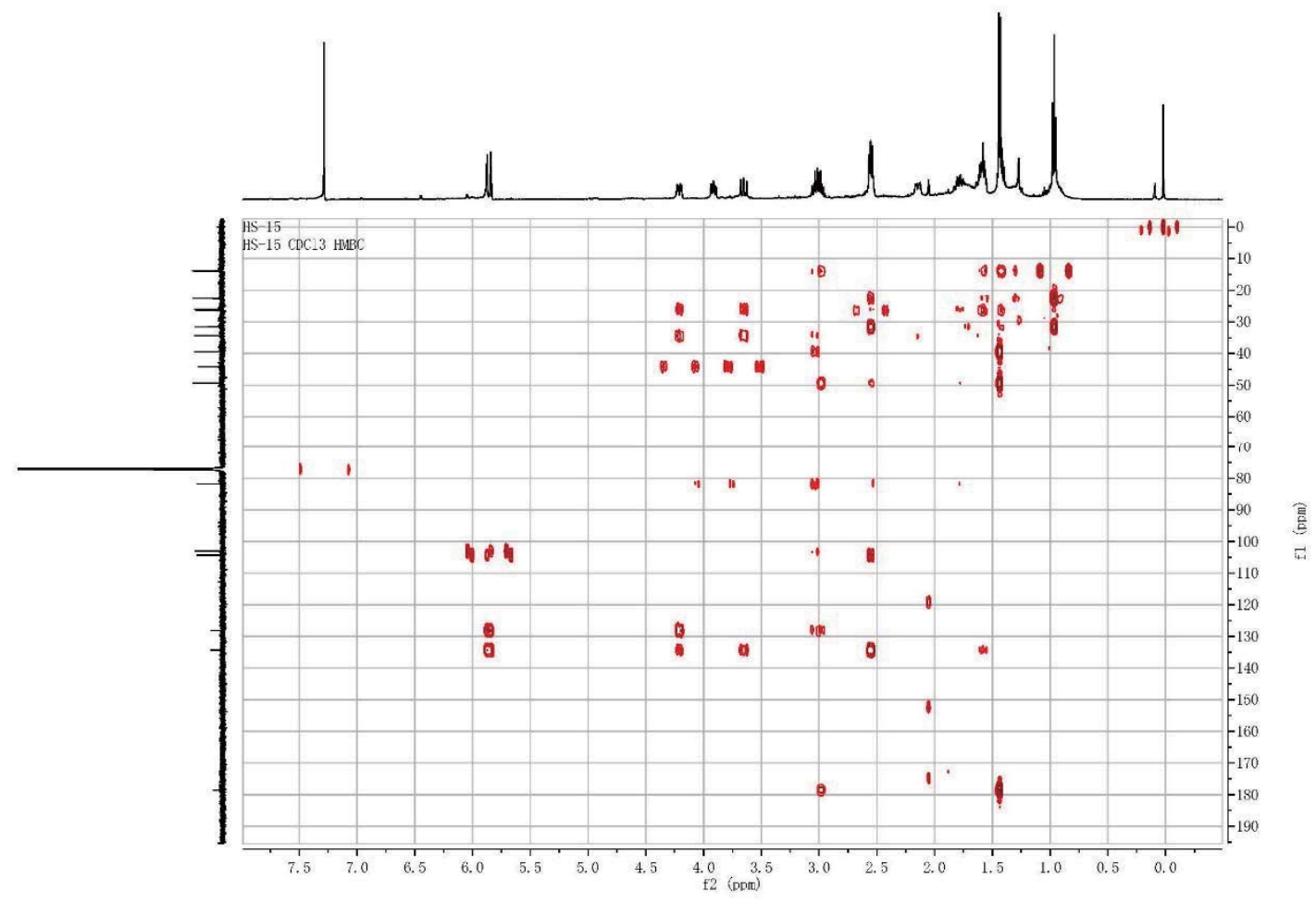


S56: ${ }^{1} \mathrm{H}{ }^{1} \mathrm{H}$ COSY of parvistemonine A (8)

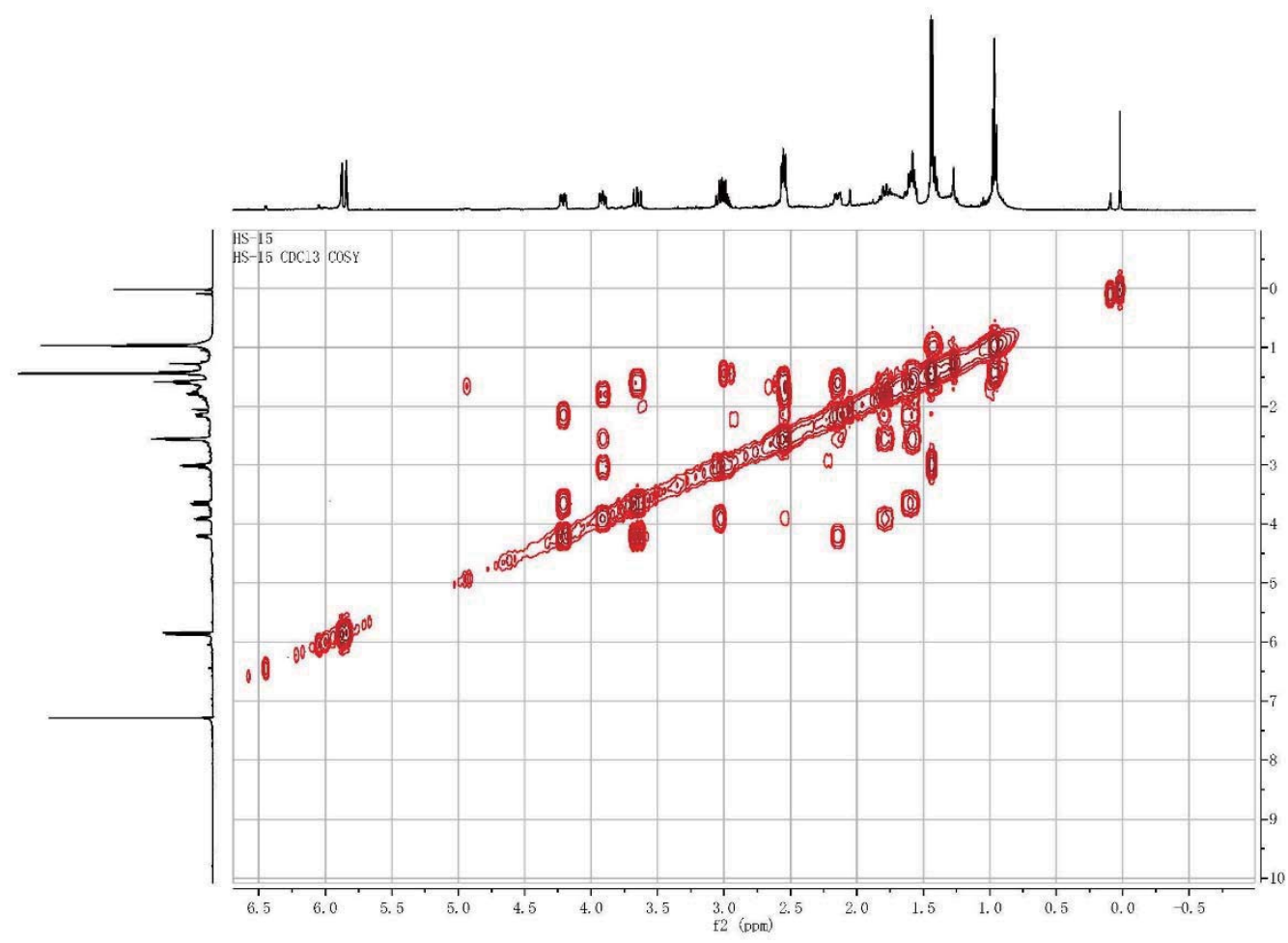

S57: ROESY of parvistemonine A (8)

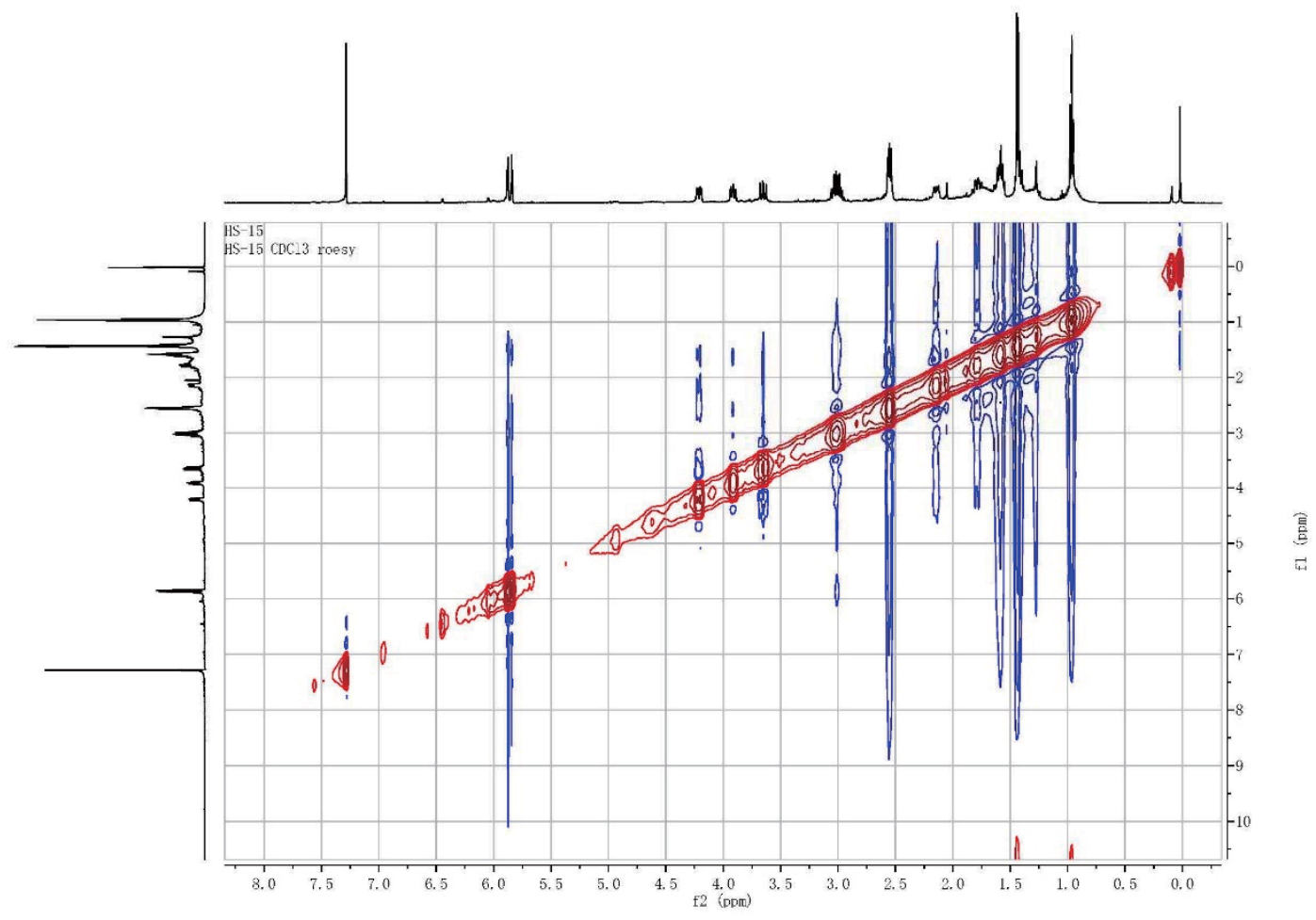


S58: ESIMS of parvistemonine A (8)

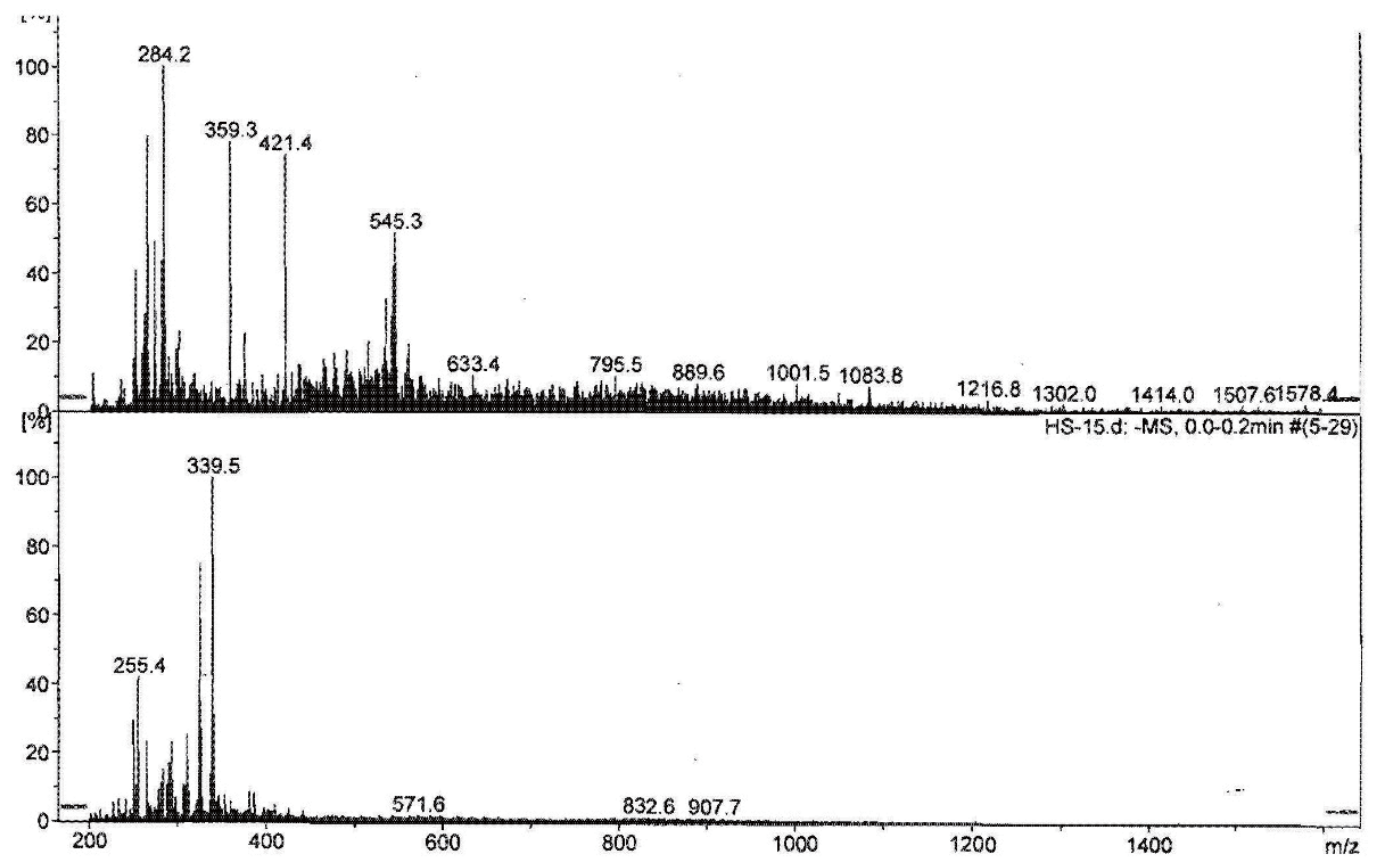

S59: CD of parvistemonine A (8)

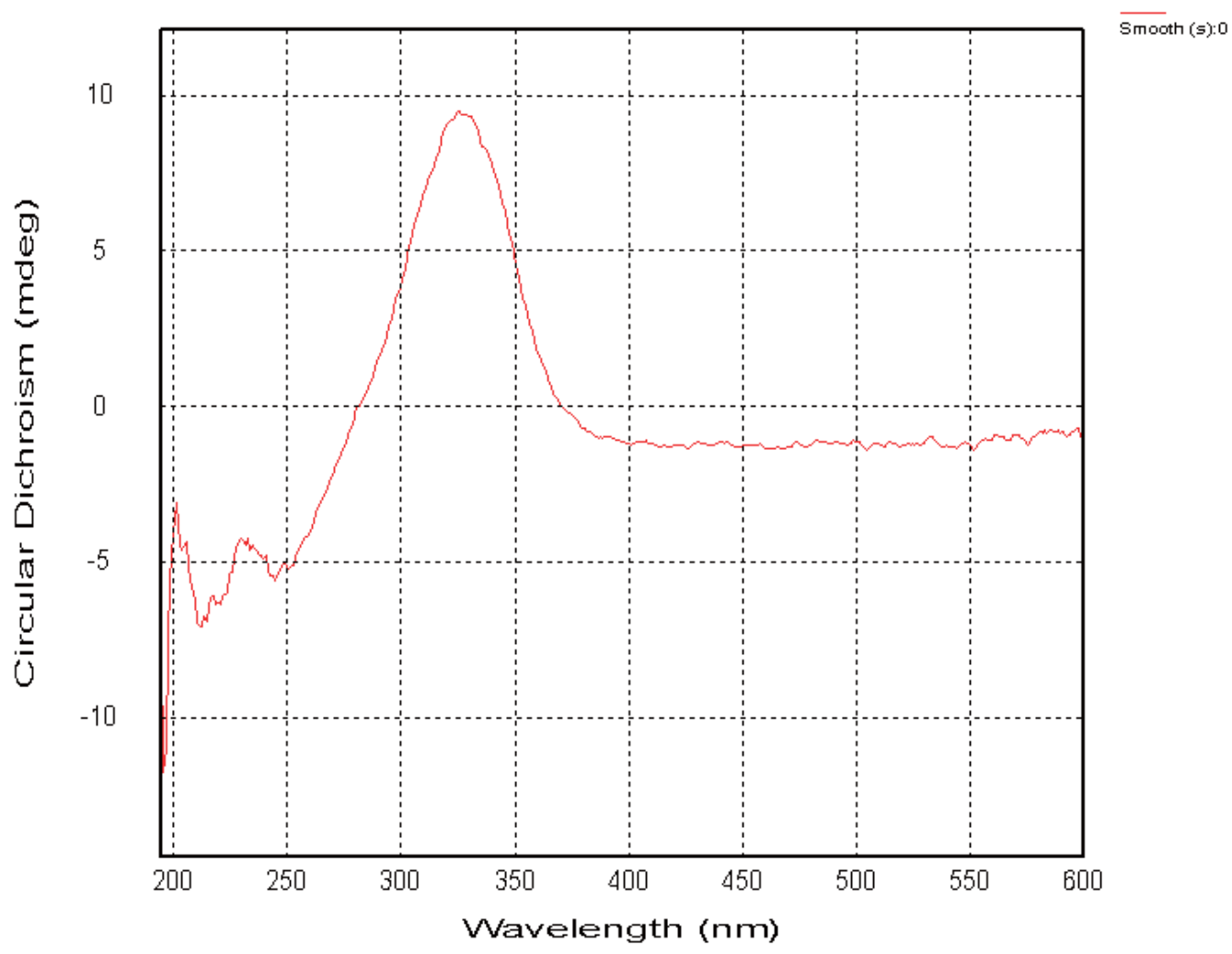

